RHIC/AP/102

June 1996

\title{
EPAC/LHC Magnet Papers
}

- G. Ambrosio, F.M. Ametrano, F. Broggi, A. Ferrari and L. Rossi, "Stability of a NbSn Low-Beta Quadrupole in the LHC Radiation Environment"

- G. Ambrosio, G. Bellomo and L. Rossi, "A 300 T/m NbSn Quadrupole for the LowBeta Insertions of the LHC"

- N. Andreev, K. Artoos, L. Bottura, G. Kirby, D. Leroy, L. Oberli, J. Ostler, D. Perini, A. Poncet, F. Rodrigues-Mateos, S. Russenschuck, T. Siambanis, N. Siegel, A. Siemko, D. Tommasini, G. Trinquart, I. Vanekov, L. Walckiers, W. Weterings, "The 1m Long Single Aperture Dipole Coil Test Program for LHC"

- R. Bossert, S. Feher, S.A. Gourlay, T. Heger, J. Kerby, M.J. Lamm, P.J. Limon, P.O. Mazur, T. Nicol, F. Nobrega, D. Orris, J.P. Ozelis, T. Peterson, P. Schlabach, J. Strait, J. Tompkins, A. Zlobin, A. Lietzke, A.D. McInturff, R. Scanlan, "Low Temperature Quench Performance of Fermilab Low- $\beta$ Insertion Quadrupoles"

- L. Bottura, A. Faus-Golfe, L. Walckiers, R. Wolf, "Field Quality of the Main Dipole Magnets for the LHC Accelerator"

- A. Faus-Golfe and A. Verdier, "Dynamic Aperture Limitations of the LHC in Physic Conditions due to Low-Beta Insertions"

- Ramesh Gupta, "Field Quality in Superconducting Magnets for Large Particle Accelerators"

- R.C. Gupta, R. Bossert, S.A. Gourlay, T. Heger, Y. Huang, J. Kerby, M.J. Lamm, P.J. Limon, P.O. Mazur, F. Nobrega, J.P. Ozelis, G. Sabbi, J. Strait, A.V. Zlobin, S. Caspi, D. Dell' orco, A.D. McInturff, R.M. Scanlan, J.M. Van Oort, "Design of a High Gradient Quadrupole for the LHC Interaction Regions"

- A. Ijspeert, J. Salminen, "Superconducting Coil Compression by Scissor Laminations"

- A. Ijspeert, R. Perin, E. Baynham, P. Clee, R. Coombs, D. Evans, M. Begg, D. Landgrebe, "Construction and Tests of a Model of the LHC Superconducting Corrector Magnet MDSBV"

- M. Karppinen, S. Russenschuck A. Ijspeert, "Automated Design of a Correction Dipole Magnet for LHC"

- N. Mokhov, J. Strait, "Optimization of the LHC Interaction Region with Respect to Beam-Induced Energy Deposition"

- R. Ostojic and T.M. Taylor, "Amplitude Dependent Tune Spread and Field Errors of Superconducting Low- $\beta$ Quadrupoles"

- J. Salminen, A. Ijspeert, A. Puntambekar, "Superconducting Sextupole Corrector Magnet for the LHC Main Dipoles" 



\title{
STABILITY OF A NbSn LOW-BETA QUADRUPOLE IN THE LHC RADIATION ENVIRONMENT
}

\author{
G. Ambrosio, F.M. Ametrano, F. Broggi, A. Ferrari and L.Rossi \\ INFN and Physics Dept. of the University of Milan , \\ LASA, via fratelli Cervi 201, 20090 Segrate (Milano) - I
}

\begin{abstract}
Use of $\mathrm{Nb}_{3} \mathrm{Sn}$ coils can significantly improve the performances of the low $\beta$ quadrupoles of hadron colliders. In this paper we report the results of a study to evaluate the average and the peak power deposition on the $\mathrm{Nb}_{3} \mathrm{Sn}$ coils of a quadrupole for the second generation of the low $\beta$ insertion for the LHC. The power release into the coils by radiation escaping from the interaction point has been investigated, by means of FLUKA code, as a function of different gradient-aperture combinations (the basic values being $300 \mathrm{~T} / \mathrm{m}-70 \mathrm{~mm}$ ). Consequently the superconducting stability of the impregnated coils has been evaluated both at 2 and $4.2 \mathrm{~K}$ operation temperatures of the magnet.
\end{abstract}

\section{INTRODUCTION}

In the frame of the CERN-INFN collaboration on superconducting magnets for the LHC, a study of a very high performance quadrupole for a second generation of inner triplet of the low $\beta$ insertions is under way at LASA lab of INFN-Milan. This type of quadrupole needs to reach very high magnetic field gradient, beyond $235 \mathrm{~T} / \mathrm{m}$ in the machine, in a single aperture bore of $70 \mathrm{~mm}$ [1].

CERN has designed and built, in collaboration with $\mathrm{Ox}$ ford Instruments (GB) [2], a full section $1.3 \mathrm{~m}$ long model -of a novel design quadrupole, that has been successfully tested up $93 \%$ of the $I_{\max }$ reaching $245 \mathrm{~T} / \mathrm{m}$ [4]. This quadrupole model makes use of very high performance NbTi cable with all kapton insulation.

To break the barrier of $250 \mathrm{~T} / \mathrm{m}$ in $70 \mathrm{~mm}$ coil aperture we have designed a quadrupole, based on $\mathrm{Nb}_{3} \mathrm{Sn}$ conductor, whose schematic cross section is reported in fig. 1. $\mathrm{Nb}_{3} \mathrm{Sn}$ has very high performance in term of $\mathbf{I}_{c}$ characteristic vs field but suffers of serious drawbacks, namely the necessity of high temperature reaction $\left(650^{\circ} \mathrm{C}\right.$ or more) and a brittleness that makes coil handling hazardous.

Brittleness of the $\mathrm{Nb}_{3} \mathrm{Sn}$ and its $\mathrm{I}_{c}$ degradation vs transverse stress in case of bare cable requires the coils be fully impregnated under vacuum after reaction. Full impregnation of the coils raises a concern about coil stability due to lack of direct cooling.

The main points on stability in our design are:

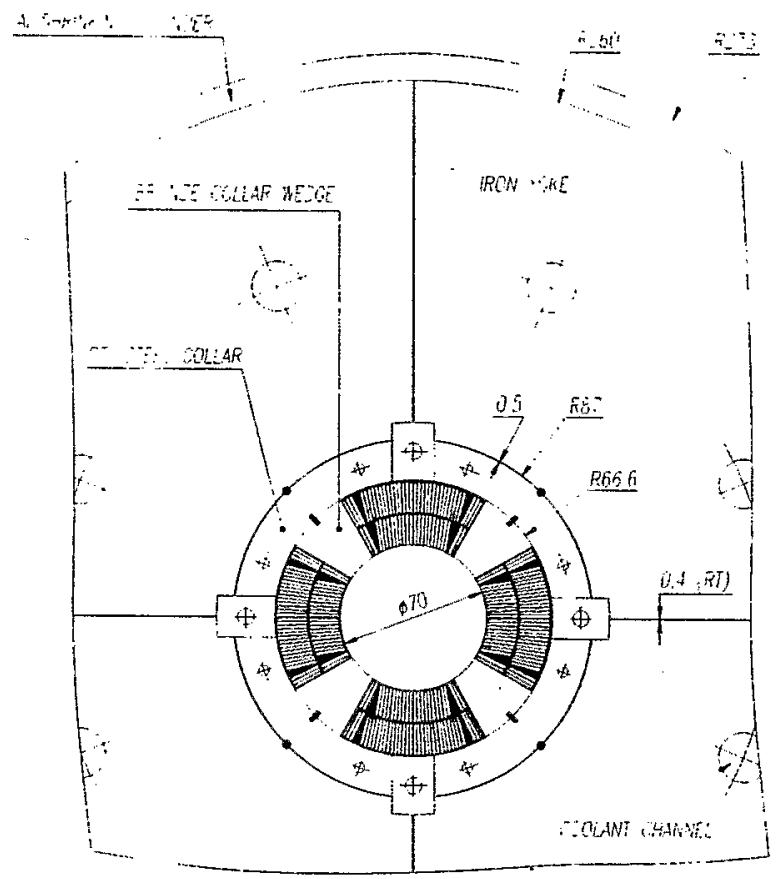

Figure 1: Schematic cross section of the $\mathrm{Nb}_{3} \mathrm{Sn}$ quadrupole.

- the peak field on the coil, $11.5 \mathrm{~T}$ at $300 \mathrm{~T} / \mathrm{m}$, imply large operating stress in the coil, over $100 \mathrm{MPa}$ in the high field region;

- impregnation of the coil will help mechanical stability on the coil head; this point is sometime negleted but it has been proved very important in the main dipole - magnet [5], such that now is part of the design of the LHC dipoles;

- when working at $1.8 \mathrm{~K}$, the disadvantage of specific heat, lower than at $4.2 \mathrm{~K}$, is not balanced by the advantage of better heat transfer;

- the stability margin can be compromized by a considerable heat deposition into the coil due to radiation which can increase significantly the operating temperature.

A study of the power deposition has been carried out by CERN [6] with a simplified model and the power deposi- 
Table 1: main features of quadrupole design

\begin{tabular}{|c|c|c|}
\hline gradie & $\begin{array}{r}85 \\
250 \\
\end{array}$ & \\
\hline $\left.\mathrm{m}^{2}\right)$ & \multicolumn{2}{|c|}{$\begin{array}{c}1.8 \\
\mathrm{Nb}_{3} \mathrm{Sn} \text { Int.Tin Diffusion } \\
1500 \text { at } 12 \mathrm{~T}, 4.2 \mathrm{~K} \\
1: 1 \\
36 \text { strands, } \phi=0.825 \mathrm{~mm} \\
1.34-1.60 \times 15.0\end{array}$} \\
\hline $\begin{array}{l}\text { opera } \\
\mathbf{J}_{\text {over }} \\
\text { peak }\end{array}$ & $\begin{array}{r}15.1 \\
565 \\
12.1 \\
\end{array}$ & $\begin{array}{r}17.9 \\
670 \\
11.45 \\
\end{array}$ \\
\hline midplane shim & \multicolumn{2}{|c|}{$\begin{array}{c}\text { R-glasstepoxy } \\
0.125 \mathrm{azim} ., 0.250 \mathrm{rad} \\
2 \times 0.3 \mathrm{~mm}\end{array}$} \\
\hline & $\begin{array}{r}3.9 \\
\leq 120 \\
\end{array}$ & $\leq 13$ \\
\hline
\end{tabular}

tion has been found to $6 \mathrm{~W} / \mathrm{m}$ ( $30 \mathrm{~W}$ per magnet). This point must be very carefully evaluted for coils which are almost adiabatic, especially because the power deposition into the coils - a substantial fraction of the total power- is strongly peaked at the coil midplanes.

\section{QUADRUPOLE CHARACTERISTICS}

We examined two designs with the same conductor: one that should generate $250 \mathrm{~T} / \mathrm{m}$ in a $85 \mathrm{~mm}$ coil aperture [7] and the latest that aims to generate $300 \mathrm{~T} / \mathrm{m}$ with $70 \mathrm{~mm}$ useful bore. The design stability margin is given by operation at $93-94 \%$ of the $I_{m a x}$ as given by short sample measurements.

The main paramenters are reported in table 1.

The load line for the $300 \mathrm{~T} / \mathrm{m}-70 \mathrm{~mm}$ case together with the $\mathrm{J}_{c \text { ov }}$ at 4.2 and $1.8 \mathrm{~K}$ for the $\mathrm{Nb}_{3} \mathrm{Sn}$ winding are reported in fig. 2, where for comparison is reported the $\mathrm{J}_{c \text { ov }}$ characteristic of the NbTi CERN-Oxford quadrupole.

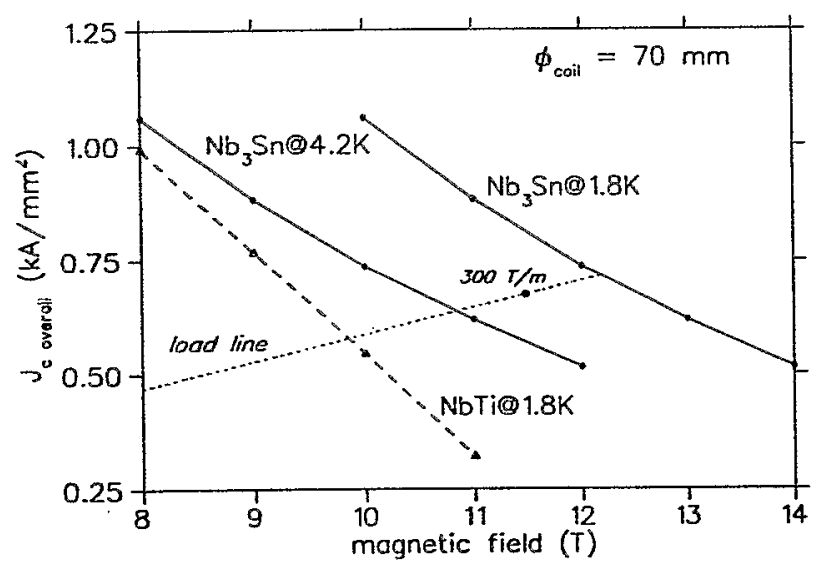

Figure 2: Coil $J_{c}$ for our $\mathrm{Nb}_{3} \mathrm{Sn}$ and for $\mathrm{NbTi}$ and magnet load line.

\section{POWER DEPOSITED BY RADIATION}

The source of the radiation power escaping from the interaction point (I.P.) has been evaluated by taking few hundreds of the $7+7 \mathrm{TeV}$ events from DTUJET code. The interesting events are the inelastic and the single diffractive ones, whose cross sections are $\sigma_{\text {in }}=60 \mathrm{mb}$ and $\sigma_{s d}=12$ $\mathrm{mb}$ respectively. The neutral particles released in the interactions were directly processed by FLUKA [9], while the charged ones were transported along the beam pipe though the detector field and through the inner quadrupole triplet of the low 3 insertion. Low energy neutrons have been neglected. Whenever a charged particle hits the beam line structure it was processed by FLUKA as a single event.

The inner triplet starts with $Q_{1}$ placed at $23 \mathrm{~m}$ from I.P., with a free space of $2.5 \mathrm{~m}$ between $Q_{1}$ and $Q_{2}, 1 \mathrm{~m}$ between $Q_{2}$ and $Q_{3}, 2.3 \mathrm{~m}$ between $Q_{3}$ and $Q_{4}$. Between the I.P. and $Q_{1}$ a $\phi_{\text {in }}=30 \mathrm{~mm}, 1.8 \mathrm{~m}$ long, copper collimator is placed at $19 \mathrm{~m}$ from I.P. Moreover the effect of four stainless steel absorbers inside the beam pipe alond the quadrupoles has been evaluated. The thickness of the absorbers is $5 \mathrm{~mm}$ [8].

The results of the calculation for the nominal luminosity of $10^{34} \mathrm{~cm}^{-2} \mathrm{~s}^{-1}$ are summarized in table 2 where data refers to $Q_{2}$ that is by far the hottest quadrupole.

The peak power deposited into the coils ranges from 1.3 (for $250 \mathrm{~T} / \mathrm{m}-85 \mathrm{~mm}$ ) up to $2.1 \mathrm{~mW} / \mathrm{cm}^{3}$ (for $300 \mathrm{~T} / \mathrm{m}$ $-70 \mathrm{~mm}$ ). We think that the grid used is too wide (about $1 \times 1 \times 50 \mathrm{~cm}^{3}$ ) and these should be regarded as minum values. For these reasons and to be conservative we preferred, in thermal analysis, to concentrate the whole power released in the coils near the midplane. In this way, in case of $300 \mathrm{~T} / \mathrm{m}-70 \mathrm{~mm}$, the power density along the midplane for the hottest coil becomes $5.8 \mathrm{~mW} / \mathrm{cm}^{3}$.

The results so far obtained are very preliminar but sufficient to evalute the stability of the $\mathrm{Nb}_{3} \mathrm{Sn}$ coil. More accurate calculation, with bigger statistic and higher number of events is underway.

\section{2-D THERMAL MODEL}

A 2-D thermal analysis was carried out by means of ANSYS code on an octant. Main features of the thermal model are:

1. steady state;

2. linear analysis, i.e. material properties are constant at the initial temperature. This hypothesis is confirmed a posteriori by the very small temperature increase;

3. regions are (iron is neglected since is thermally separated):

(a) two coil shells, each one containing a longitudinal copper wedge, where the material properties are the effective values of the conductor unit cell; typical mesh dimension in the coil is $2 \times 2 \mathrm{~mm}^{2}$; 
Table 2: heat deposition (four poles) and coil temperature rise above $2 \mathrm{~K}$

\begin{tabular}{|l|c|c|c|c|c|c|}
\hline $\begin{array}{c}\text { apert. } \times \text { grad. } \\
(\mathrm{mm} \times \mathrm{T} / \mathrm{m})\end{array}$ & $\begin{array}{c}\text { power into } \\
\text { coils }(\mathrm{W} / \mathrm{m})\end{array}$ & $\begin{array}{c}\text { hottest } \\
\text { coil }(\mathrm{W} / \mathrm{m})\end{array}$ & $\begin{array}{c}\text { power in } \\
\text { th. calc. }\left(\mathrm{mW} / \mathrm{cm}^{3}\right)\end{array}$ & $\begin{array}{c}\Delta \mathrm{T}_{\max } \\
\text { coils }(\mathrm{K})\end{array}$ & $\begin{array}{c}\text { total power into } \\
\text { cold mass }(\mathrm{W} / \mathrm{m})\end{array}$ & $\begin{array}{c}\text { power into } \\
\text { adsorber }(\mathrm{W} / \mathrm{m})\end{array}$ \\
\hline $70 \times 250$ & 1.27 & 0.36 & 4.7 & 0.42 & 3.84 & 2.31 \\
$70 \times 300$ & 1.44 & 0.44 & 5.8 & 0.50 & 4.30 & 2.56 \\
$85 \times 250$ & 1.27 & 0.35 & 4.6 & 0.42 & 3.98 & 2.42 \\
\hline
\end{tabular}

Table 3: stability margin of the coils (without radiation)

\begin{tabular}{|l|c|c|r|}
\hline apert. $\times$ grad. & $\mathrm{T}_{\text {batt }}(\mathrm{K})$ & $\Delta \mathrm{T}_{m}(\mathrm{~K})$ & $\Delta \mathrm{H}_{m}\left(\mathrm{~kJ} / \mathrm{m}^{3}\right)$ \\
$70 \times 300$ & 2.0 & 2.8 & 5.24 \\
$70 \times 300$ & 4.2 & 2.4 & 11.3 \\
\hline
\end{tabular}

(b) radial insulation regions on the inner coil raius, between two coil shells and between outer coil radius and collar;

(c) pole wedge in bronze and, separated, the insulation between pole wedge and coils;

(d) stainless steel ring collar;

4. no heat exchange at midplane and along the $45^{\circ}$ boundaries (symmetry conditions); convective heat exchange with cryogen, $\mathrm{HeII}$ or $\mathrm{LHe}$, at fixed temperature: a conservative heat transfer coefficient of $100 \mathrm{~W} / \mathrm{m}^{2}-\mathrm{K}$ has been taken.

5. the total power released into the coil has been concentrated in the mesh elements along the midplane; computation has been done for the coil with the biggest heat deposition;

The inner bound insulation region has been magnified in radial dimension to be properly described and consequently its thermal conductivity amplified by the same factor.

The results of this preliminary analysis, computed at $2 \mathrm{~K}$ where the thermal conductivity are worse than in the case of $4.2 \mathrm{~K}$, are summarized in the table 2 .

The heat flow to the helium is very low, with a peak of about $17.7 \mathrm{~W} / \mathrm{m}^{2}$, generating a temperature rise of $0.17 \mathrm{~K}$ at the coil to helium interface.

\section{STABILITY ANALYSIS}

The numbers reported in the previuos table must be compared with the stability margin for the coil operation. The stability can be reported as temperature margin, $\Delta T_{m}$, or enthalpy margin (against distributed continuous perturbation), $\Delta \mathbf{H}_{m}$. These values are reported in table 3 without taking into account the radiation heat.

It can be seen on the temperature margin that the $0.5 \mathrm{~K}$ of temperature increase due to heat depostion, see table 2, has a small effect on the coil stability. Actually the first $0.5 \mathrm{~K}$ above $2 \mathrm{~K}$ gives negligible contribution to enthalpy margin which decreases from 5.24 down to $4.8 \mathrm{~kJ} / \mathrm{m}^{3}$. The situation is even better at $4.2 \mathrm{~K}$ where the residual $1.9 \mathrm{~K}$ temperature margin corresponds to a more comfortable 9.8 $\mathrm{kJ} / \mathrm{m}^{3}$ enthalpy margin.

\section{CONCLUSIONS}

This preliminary calculation shows that $\mathrm{Nb}_{3} \mathrm{Sn}$ technology can be employed for these very demanding magnets. From the point of view of stability the heat deposition by radiation is acceptable without endangering the low $\beta$ quadrupole operation also at superfluid temperature.

\section{ACKNOWLEDGEMENTS}

Authors gratefully acknowledge G. Kirby, R. Ostojic and J. Strait of CERN for the useful discussion and information provided.

\section{REFERENCES}

[1] LHC - conceptual design, edited by P. Lefe vre and T. Pettersson, report CERN/AC/95-05(LHC), 1995.

[2] R.Ostojic, T.M.Taylor, G.A.Kirby, "Design and Construction of a One-Metre Model of the $70 \mathrm{~mm}$ Aperture Quadrupole for the LHC Low- $\beta$ Insertions", Proc. of MT13, Vancouver B.C. 1993, pag 1750.

[3] G. Ambrosio, G. Bellomo and L. Rossi, "Study of a High Gradient, Large Aperture, $\mathrm{Nb}_{3} \mathrm{Sn}$ Quadrupole for the low $\beta$ Insertions of the LHC", Proc. of EPAC94, London, pag 2268.

[4] T. Taylor, CERN-LHC div,, private communication, 1996.

[5] M.Bona, R.Perin, E.Acerbi and L.Rossi “Assessment of the Test Results of the Two CERN-INFN $10 \mathrm{~m}$ Long Superconducting Dipole Prototypes for the LHC". Proc. of MT-14 Conf., Tampere (Finland) 1995.

[6] A. Morsch, R. Ostojic and T.M. Taylor, "Progress in the System Design of the Inner Triplet of $70 \mathrm{~mm}$ Aperture Quadrupoles for the LHC Low- $\beta$ Insertions", Proc. of EPAC94, London, pag 2274.

[7] G. Ambrosio et al., "Preliminary Proposal of a $\mathrm{Nb}_{3} \mathrm{Sn}$ Quadrupole Model for the low $\beta$ insertions of the LHC", report INFN/TC-95/25, 1995.

[8] R. Ostojic, CERN-LHC div., private communication.

[9] A. Fasso, A. Ferrari, J. Ranft and P. R. Sala, "FLUKA: present status and future developments", Proceedings of the IV International Conference on Calorimetry in High Energy Physics, La Biodola (Elba), September 19-25 1993, A. Menzione and A. Scribano eds., World Scientific, pag 493 (1994). 
$$
\text { - }
$$ 


\title{
A 300 T/m NbSn QUADRUPOLE FOR THE LOW-BETA INSERTIONS OF THE LHC
}

\author{
G. Ambrosio, G. Bellomo and L. Rossi \\ INFN and Physics Dept. of the University of Milan, \\ LASA, Via Fratelli Cervi 201, 20090 Segrate (Milano) - I
}

\begin{abstract}
The NbTi quadrupoles for the LHC insertions have a design gradient of $250 \mathrm{~T} / \mathrm{m}$ at $1.8 \mathrm{~K}$ with a $70 \mathrm{~mm}$ aperture. We are exploring the use of the $\mathrm{Nb}_{3} \mathrm{Sn}$ technology for second generation low $\beta$ quadrupoles for LHC. A conceptual design of a $\mathrm{Nb}_{3} \mathrm{Sn}$ quadrupole operating at $1.8 \mathrm{~K}$ with 300 $\mathrm{T} / \mathrm{m}$ gradient and $70 \mathrm{~mm}$ aperture is presented. Cable performance, winding technique,magnetic design, mechanical structure and magnet protection are discussed.
\end{abstract}

\section{INTRODUCTION}

In the LHC (Large Hadron Collider) the proton beams are focussed at each side of the IP (Interaction Point) by a set of four quadrupoles, series connected, forming the inner triplet. These quadrupoles, $5.5 \mathrm{~m}$ long, have single aperture $(70 \mathrm{~mm}$ ) and a design gradient of $250 \mathrm{~T} / \mathrm{m}$ at $1.8 \mathrm{~K}$.

A $1.3 \mathrm{~m}$ long model using an high quality NbTi conductor and a novel design [1] has been built by a CERN -Oxford Instruments (GB) collaboration and has reached a gradient of $245 \mathrm{~T} / \mathrm{m}$.

In the frame of a collaboration between CERN and INFN on superconducting magnets we have looked to alternative design using $\mathrm{Nb}_{3} \mathrm{Sn}$ conductor. An initial investigation [2], based on simple scaling laws and assuming as a reference the CERN-Oxford design, has explored various layout (shell or rectangular block) and different bore apertures ( 70 or $85 \mathrm{~mm}$ ) indicating the possibility to reach with the $\mathrm{Nb}_{3} \mathrm{Sn}$ technology the $300 \mathrm{~T} / \mathrm{m}$ with the small aperture or $250 \mathrm{~T} / \mathrm{m}$ with the large bore.

A more detailed study [3], including the mechanical structure and magnet protection, has been completed for a large aperture $(85 \mathrm{~mm})$ quadrupole. Finally the choice has been made to maintain the nominal aperture $(70 \mathrm{~mm})$ and to aim at gradient of $300 \mathrm{~T} / \mathrm{m}$. In this paper the conceptual design of this quadrupole is presented and the relevant aspects are discussed in detail.

\section{CONDUCTOR}

We plan to use a conductor manufactured by Europa Metalli (Florence) with the Internal Tin Diffusion method. It has 37 islands embedded in a copper matrix. Each island, which has a $\mathrm{Sn}$ core and $190 \mathrm{Nb}$ filaments, is enclosed by a $\mathrm{Nb}$ barrier, whose thickness is about $20 \mu \mathrm{m}$, to prevent poisoning of the pure copper during heat treatment. The barrier is partially reacted into $\mathrm{Nb}_{3} \mathrm{Sn}$ : this helps to obtain
Table 1: Superconducting cable.

\begin{tabular}{|l|l|}
\hline superconductor & $\mathrm{Nb}_{3} \mathrm{Sn}$ - Int. Tin Diffusion \\
barrier & niobium \\
effective $\phi_{f i l}$ & $20-30 \mu \mathrm{m}$ \\
$\phi$ strand & $0.825 \mathrm{~mm}$ \\
$\alpha=\mathrm{Cu}$ :non $\mathrm{Cu}$ & 1 \\
heat treat. temp. & up to $650^{\circ} \mathrm{C}, 350$ hours \\
$\mathrm{J}_{c}$ non $\mathrm{Cu}$ & $1500 \mathrm{~A} / \mathrm{mm}^{2} @ 12 \mathrm{~T}, 4.2 \mathrm{~K}$ \\
n. of strands & 36 \\
cable size & $1.34 \div 1.60 \times 15.0 \mathrm{~mm}^{2}$ \\
\hline
\end{tabular}

very high $\mathrm{I}_{c}$ values but is detrimental for the effective filament diameter that ranges from 20 to $30 \mu \mathrm{m}$, depending on the layout [4].

The $\mathrm{Nb}_{3} \mathrm{Sn}$ critical current density measured in virgin wires at $4.2 \mathrm{~K}$ vs applied magnetic field is reported in Fig 1 together with the scaling to $1.8 \mathrm{~K}$. Also shown, for comparison, is the NbTi curve of the conductor used in the CERNOxford quadrupole. The cable characteristics are reported in Table 1 .

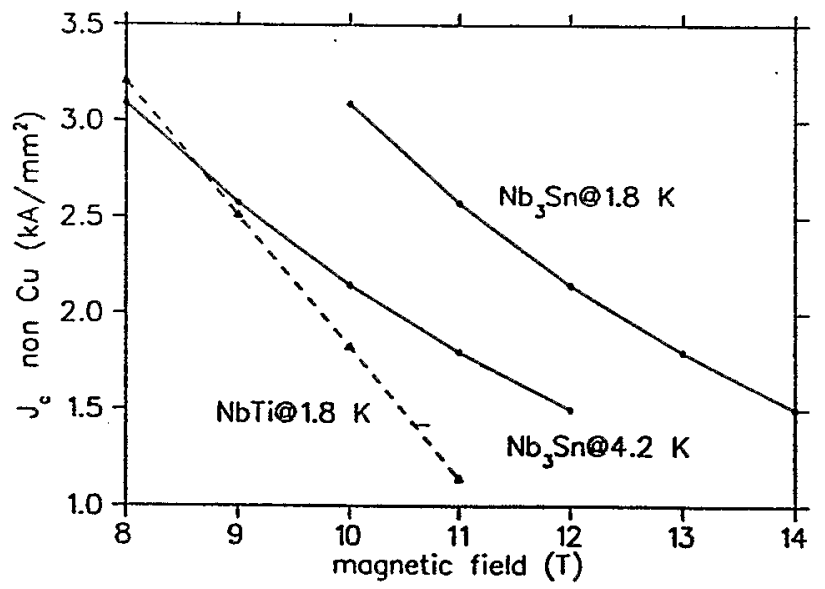

Figure 1: Non copper critical current density for $\mathrm{Nb}_{3} \mathrm{Sn}$ and $\mathrm{NbTi}$ in virgin wires.

Disadvantages of $\mathrm{Nb}_{3} \mathrm{Sn}$ technology with respect to the more standard NbTi one have been extensively described in [3]. In the meanwhile we have investigated $\mathrm{I}_{c}$ degradation due to cabling. In a prototype cable, $150 \mathrm{~m}$ long and with $7 \%$ lower current density, degradation after cabling (measured on wires) was found about $5 \%$, very near to the stan- 
dard $3 \%$ for good NbTi cable. Also the $\mathrm{L}_{c}$ degradation vs transverse compression, a key factor for this type of magnet, will be soon measured.

To avoid degradation of the outer copper quality and possible flake off of the barrier during heat treatment due to oxygen contamination through the thin copper layer, our conductor must be treated under vacuum or inert atmosphere during all the stages of reaction.

\section{INSULATION AND WINDING}

The coils are wound and then reacted. So far we have tested for insulation three different materials: R-glass tape, micaglass tape from Isovolta (A) and a ceramic-glass composite foil from Toshiba (J). Each insulation has been treated at high temperature $\left(700^{\circ} \mathrm{C}\right)$ in Ar atmosphere and under vacuum in shape of tape or foil and then the insulation resistance has been measured up to $1 \mathrm{kV}$ under a pressure of over $50 \mathrm{MPa}$.

Results have been very positive for the mica-glass tape and for the ceramic-glass foil, with $R \geq 10 \mathrm{M} \Omega$ at $1 \mathrm{kV}$ and $\geq 1 \mathrm{G} \Omega$ at $500 \mathrm{~V}$. The R-glass tape has shown,after a special chemical treatment [5] to remove the sizing agent,insulation resistance of typically $1 \mathrm{M} \Omega$ at $1 \mathrm{kV}$ and $\geq 1 \mathrm{G} \Omega$ at $500 \mathrm{~V}$. It should be mentioned that resistance on glass samples occasionally is much lower and that the chemical process is not yet tested on a real coil. We rely also on the fact that continuity of the insulation should be assured by proper impregnation of the coil under vacuum. For the time being we keep as interturn insulation a R-glass tape, that is easy available in different dimensions, butt-lap wrapped around the cable and enclosing, on one side only of the cable, a further glass tape. In this way the turn to turn gap is $240 \mu \mathrm{m}$.

The alternatives under study are:

i) use of glass tape wrapped around the cable and enclosing a mica-glass tape (probably it is the best solution for insulation),

ii) ceramic-glass tape used only as a spacer, if it will be available in the proper dimension. In this way the turn to turn distance is $150 \mu \mathrm{m}$.

The ceramic-glass tape is more robust than R-glass and more flexible than mica-glass but unfortunately the minimum available thickness is $150 \mu \mathrm{m}$ while R-glass is 80 $\mu \mathrm{m}$ thick and mica-glass is $100 \mu \mathrm{m}$.

The coils are wound in double pancake technique to avoid the joint between the inner and outer layer of each pole which is normally a weak point of the magnet and can limit the performance.

\section{COIL LAYOUT}

The double pancake technique prevents the possibility of grading ; we point out however that the grading is less effective in $\mathrm{Nb}_{3} \mathrm{Sn}$ coils respect to $\mathrm{NbTi}$ because of the reduced slope of the Jc vs B line ( see fig 1). No specific cable optimization has been pursued ; we selected actually
Table 2: Quadrupole characteristics.

\begin{tabular}{|l|c|c|}
\hline Operating gradient & $(\mathrm{T} / \mathrm{m})$ & 300 \\
Load line margin & & $94 \%$ \\
Peak field in conductor & $(\mathrm{T})$ & 11.5 \\
Operating current & $(\mathrm{kA})$ & 17.9 \\
$\left|b_{6}\right| \cdot\left|b_{10}\right|$ & $\left(10^{-4}\right.$ units $)$ & $<0.01$ \\
No. of turns per coil & & $12+17$ \\
Inner/outer coil diameter & $(\mathrm{mm})$ & $70 / 132.2$ \\
Inner/outer yoke diameter & $(\mathrm{mm})$ & $174 / 520$ \\
Stored energy & $(\mathrm{kJ} / \mathrm{m})$ & 453 \\
Inductance & $(\mathrm{mH} / \mathrm{m})$ & 2.8 \\
\hline
\end{tabular}

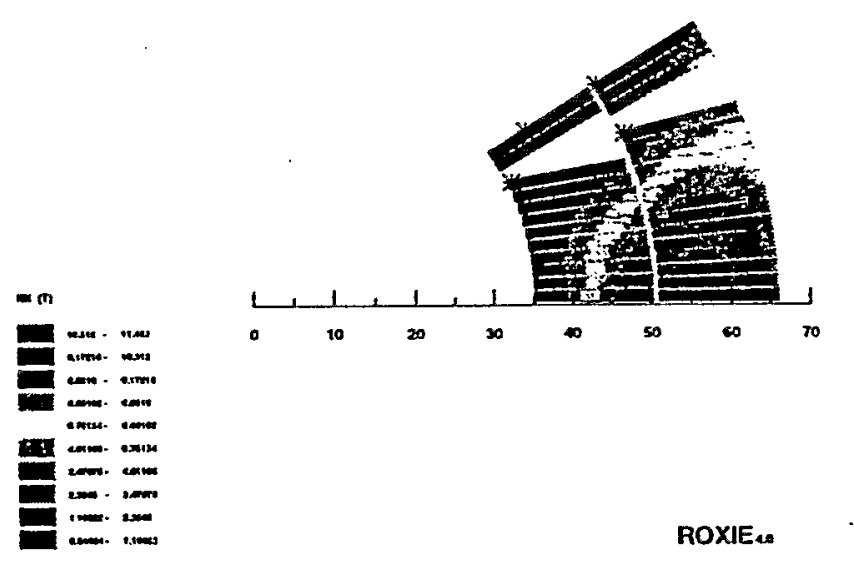

Figure 2: Coil layout (one octant) with field distribution. Peak field locations for each block are indicated.

strand diameter, cable size and keystone angle corresponding to the outer layer of the LHC dipole in order to avoid any cabling development.

The cable layout, calculated with ROXIE [6], is presented in fig 2 and the main parameters of the magnet design are given in Table 2. The $300 \mathrm{~T} / \mathrm{m}$ gradient corresponds to $94 \%$ of the load line; so far no degradation for the azimuthal stress (110 MPa) has been considered pending measurements. The iron yoke is placed at $20 \mathrm{~mm}$ from the coil in order to have a rigid collars; an iron closer to the coils, $10 \mathrm{~mm}$, gives only $1.4 \%$ increase in gradient. The operating current is large, $17.9 \mathrm{kA}$, but still manageable, to get the advantage of a low inductance which makes the protection of the magnet easier.

\section{MECHANICAL STRUCTURE}

The main characteristics of the mechanical structure,schematically shown in fig 3 are:

i) a $20 \mathrm{~mm}$ stainless steel collar ring and four bronze wedges in the pole regions;

ii) an iron yoke, $174 \mathrm{~mm}$ thick, cut in four parts and in contact with the collar only near the coil midplanes;

iii) an aluminum shrinking cylinder $13 \mathrm{~mm}$ thick.

The mechanical structure has been designed to mini- 
mize the transverse compression in order to reduce the critical current degradation. The mechanical separation of the pole wedge from the ring collars allows to keep the wedge always in position from winding to yoking. The collar wedges are made out of bronze to fit the thermal contraction of the coil during the cooldown. Materials with a lower thermal contraction, like Ti alloys, give a further prestress during cooldown, and also longitudinal stress on coil heads, but they cause an elliptical deformation of coils increasing the peak stress during magnet excitation. Material and thickness of the collar ring have been designed to withstand the magnetic forces with negligible deformation.

Almost all coil prestress is given during collaring and the force nedeed is about 500 ton/m using a dipole type press. Two collaring techniques are under study: the first using a special press[3] acting simultaneously on X,Y planes, the second using clamps for the precollaring and then a dipole type press acting alternatively on the two planes and inserting pinning rods of increasing diameter. This last technique requires an expandable collaring mandrel.

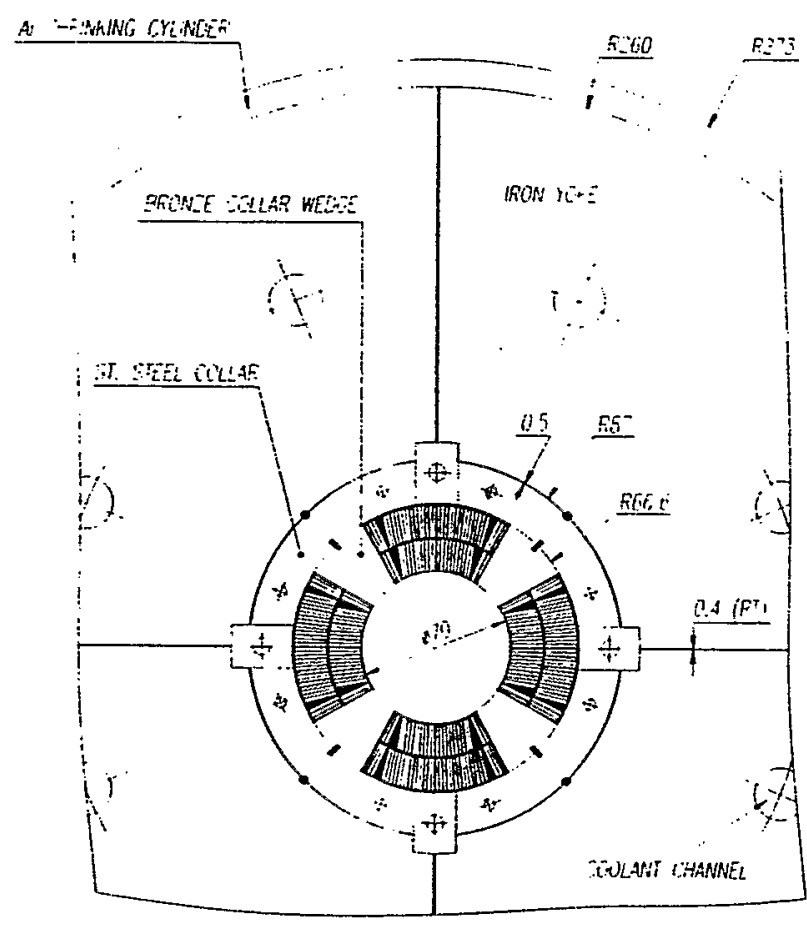

Figure 3: Cross section of the magnet

The yoke and the shrinking cylinder have been designed in order to minimize the coil stress and the coil deformation at full magnet excitation. In the first studies, trying different collar materials and thicknesses, the peak pressure in the coils was always found in the midplane at the inner side of the first layer (as usual in this type of magnet) with values between 140 and $160 \mathrm{MPa}$. This peak was due to the e.m. forces $\left(F_{r}=93 \mathrm{ton} / \mathrm{m}, F_{\theta}=-289\right.$ ton $/ \mathrm{m}$ in each octant) and to a deformation of the collar that give a $\mathbf{0 . 0 6}$ $\mathrm{mm}$ deformation of the inner edges of the coils.

In this design the shrinking cylinder gives after coldown a force of $100 \mathrm{ton} / \mathrm{m}$ for octant that the yoke transfers to the collar only on the midplanes, causing an opposite deformation. In this way when the magnet is at maximum excitation this deformation is almost all compensated, the peak stress value (123 MPa) is still on the midplane but on the outer edge of the coil (where the field is much lower) while on the inner edge the peak stress is less than $110 \mathrm{MPa}$.

\section{STABILITY AND PROTECTION}

A preliminary evaluation [7] of the stability problem of the coils at $1.8 \mathrm{~K}$ with a peak power deposition by radiation of $2.1 \mathrm{~mW} / \mathrm{cm}^{3}$ indicates that impregnated coils will have a sound margin of stability, $1.9 \mathrm{~K}$ above the operating temperature.

The magnet can be protected with an active heaters system, as in the LHC dipoles. A passive system based on a dumping resistor can be used for a $1 \mathrm{~m}$ long model[3]. The extension of the passive system to a full length magnet is under investigation.

\section{CONCLUSION}

A $70 \mathrm{~mm}$ bore $\mathrm{Nb}_{3} \mathrm{Sn}$ quadrupole with a gradient of 300 $\mathrm{T} / \mathrm{m}$ at $1.8 \mathrm{~K}$ seems feasible with the present state of the art and without major cable improving.

A proposal will be submitted to INFN In 1996 to build and test a $1 \mathrm{~m}$ long model.

Test on insulation and critical current degradation under stress are under way. A small racetrack coil ( 12 turns, 0.4 $\mathrm{m}$ long) is under construction to verify all technical aspects of insulation, winding and impregnation.

\section{ACKNOWLEDGEMENTS}

We wish to thank T. Taylor, R. Ostojic and S. Russenschuck of CERN for fruitful discussions.

\section{REFERENCES}

[1] R. Ostojic, T.M. Taylor, G.A. Kirby, " Design and Construction of One-Meter Model of the $70 \mathrm{~mm}$ Aperture Quadrupole for the LHC Low- $\beta$ Insertions", CERN AT/93-36(MA) and Proc. of EPAC94, London 1994, pag 2271.

[2] G. Ambrosio,G. Bellomo and L.. Rossi, "Study of a High Gradient,Large Aperture $\mathrm{Nb}_{3} \mathrm{Sn}$ Quadrupole for Low- $\beta$ Insertions", Proc. of EPAC, Londra 1994, pag 2268.

[3] G. Ambrosio et al , "Preliminary Proposal of a $\mathrm{Nb}_{3} \mathrm{Sn}$ Quadrupole Model for the Low- $\beta$ Insertionsof the LHC" Intemal Report INFN/TC-95/25 (1995)

[4] R. Garre' ( Europa Metalli) - private communication

[5] proprietary process of Ansaldo Energia.Genova.-I

[6] S. Russenschuck, “ROXIE" ,Report CERN AT/93-27 MA (1993)

[7] G. Ambrosio et al , "Stability of NbSn Low- $\beta$ Quadrupole in the LHC radiation environment", this conference. 



\title{
THE 1m LONG SINGLE APERTURE DIPOLE COIL TEST PROGRAM FOR LHC
}

\author{
N.Andreev, K.Artoos, L.Bottura, G.Kirby, D. Leroy, L. Oberli, J. Ostler, D.Perini, A.Poncet, \\ F.Rodriguez-Mateos, S.Russenschuck, T.Siambanis, N.Siegel, A.Siemko, D.Tommasini, \\ G.Trinquart, I.Vanenkov, L. Walckiers, W.Weterings, CERN, Geneva, Switzerland
}

\begin{abstract}
The CERN short model activity on main dipole magnets is centred around the design, in-house fabrication and testing of single and twin aperture $1 \mathrm{~m}$ long magnets. In order to study the influence of individual coil parameters on the magnet behaviour with a fast turn around rate and to qualify the possible design solutions priority was given to the fabrication of a certain number of single aperture dipole models. The collared coils are assembled in a reusable yoke structure and tested in a vertical cryostat at $2 \mathrm{~K}$. The present paper reviews the aims of the program, the design and fabrication to date of single aperture models, their instrumentation and the preliminary results and conclusions.
\end{abstract}

\section{INTRODUCTION}

The Large Hadron Collider (LHC) [1], approved by the CERN Council in December 1994, is a $7 \mathrm{TeV}$ proton accelerator-collider operating at a nominal field of $8.3 \mathrm{~T}$. Its main components are double aperture superconducting dipole magnets which have evolved for reasons of economy and machine requirements from 10 to $15 \mathrm{~m}$ in length, 50 to $56 \mathrm{~mm}$ in bore diametre, and 17 to $15 \mathrm{~mm}$ in cable width [2]. Of the former designs seven $10 \mathrm{~m}$ long double aperture dipoles and 14 short $1 \mathrm{~m}$ models (both single and double aperture with constructional variants) were built and tested, totalling nearly 70 test campaigns at cryogenic temperatures [3]. Results show that these magnets have similar performance and training behaviour, exhibiting comparable weak spots located especially in the coil ends, jump/splice regions, transitions of cross section and often in the innermost coil turn. Since improvements and design options can conveniently be studied with short magnets an intensive fabrication program of $1 \mathrm{~m}$ models of the new design started at CERN to provide the required input to the long magnet program presently in progress in industry.

\section{AIMS OF THE MODEL PROGRAM}

Priority was given to the fabrication, collaring and testing of model coils in a single aperture structure (MBSMS) to optimise specific components and procedures, implement new design features, check expected performances and provide a facility for testing cable performance and possible variants. Present rate of model completion is about one per month with a lead time of 3 to 4 months. This will allow in due time, to incorporate in the design lessons learned from the cold test results, to refine the assembly techniques and to accumulate statistics. Double aperture models will be built using the same collars and yoke laminations as for the long magnets. The first such model is planned for measurements in early autumn of this year.

\section{DESIGN AND FABRICATION}

The cross-section and main parameters of MBSMS models are shown in Fig. 1 and Table 1 below.

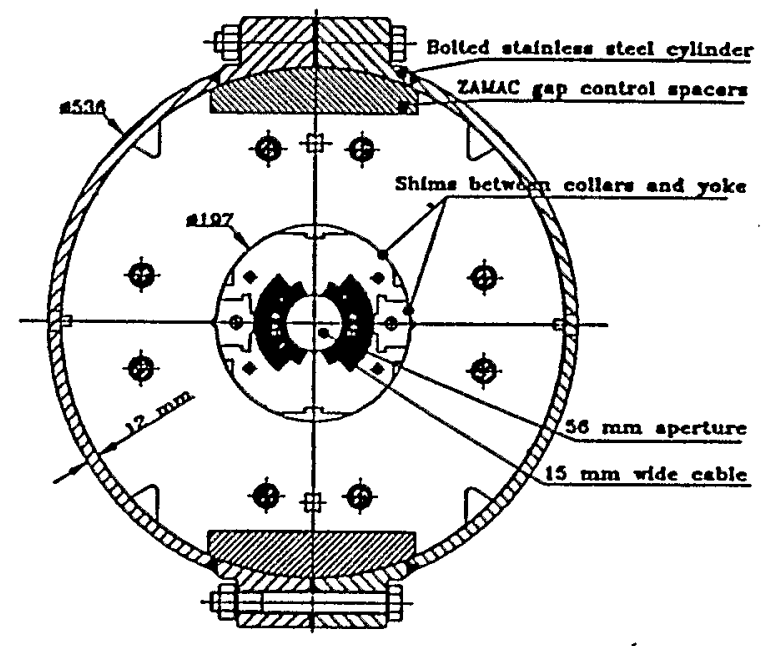

Fig. 1: Cross section of MBSMS type model.

\begin{tabular}{|l|l|}
\hline \multicolumn{2}{|c|}{ Table 1. Main design parameters } \\
\hline Coil inner diameter & $56 \mathrm{~mm}$ \\
Quenching field & $9.6 \mathrm{~T} @ 1.9 \mathrm{~K}$ and $13240 \mathrm{~A}$ \\
Nominal current (Inom) @ 8.368T & $11460 \mathrm{~A}$ \\
Ratio of peak field to central field & 1.05 \\
Overall coil length & $1080 \mathrm{~mm}$ \\
Length of magnetic steel in the yoke & $560 \mathrm{~mm}$ \\
Magnetic length & $862 \mathrm{~mm}$ \\
Total inductance & $3.2 \mathrm{mH}$ \\
Magnetic forces per quadrant @ Inom & $\sum \mathrm{Fx}=1650 \mathrm{~N} / \mathrm{mm}$ \\
& $\sum \mathrm{Fy}=-820 \mathrm{~N} / \mathrm{mm}$ \\
Total axial force @ Inom & 19 tons \\
Cu/SC ratio of inner / outer strands & $1.60 / 1.90$ \\
Ic / dic/dB of inner cable @ 1.9 K. 10 T & $213.75 \mathrm{kA} / 4.8 \mathrm{kATT}$ \\
Ic/dlc/dB of outer cable @ 1.9 K.9T & $212.95 \mathrm{kA} / 3.65 \mathrm{kA} / \mathrm{T}$ \\
\hline
\end{tabular}




\subsection{Magnetic design}

The coils have two layers, made of graded cables with 28 strands of $1.065 \mathrm{~mm}$ diameter for the inner layer and with 36 strands of $0.825 \mathrm{~mm}$ for the outer layer. The conductors are distributed in five blocks giving the required low content of $b 7$ and $b 9$ components. The load line and expected quench fields calculated with the programs ROXIE and POISOPT are shown in Fig. 2. Three basic types of coil end geometries were designed (see Table 2). Around both coil ends the yoke is made of non-magnetic laminations to reduce the peak field in these critical regions.

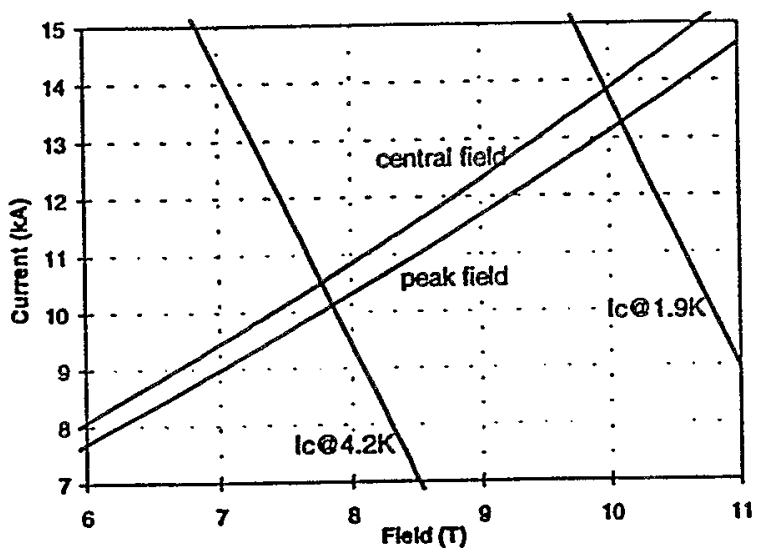

Figure 2. Load line for MBSMS magnets.

\subsection{Mechanical design}

The MBSMS design concept [4] is to allow reproducible coil testing conditions. The collars, made of Alalloy type 5083, are of similar mechanical rigidity as adopted for the double aperture design and locked by stainless steel rods. The yoke is vertically split with an open gap at room temperature, controlled by spacers made of $\mathrm{Zn} / \mathrm{Al}$ alloy, and held together by an outer stainless steel bolted shrinking cylinder which has an azimuthal pre-stress of $150 \mathrm{MPa}$ at ambient temperature. This design permits an easy assembly and reuse of the yoke structure. The gap control spacers limit coil compression during assembly but shrink away at cold so that under nominal conditions the gap is closed and the collars are just in contact with the yoke laminations. The gap remains closed up to 9.7 . T. The design azimuthal coil pre-stress after assembly at room temperature is 50 $\mathrm{MPa}$, both for inner and outer layers. Since longitudinal compression of the coil heads may improve their training performance, so-called end-cages consisting of a glued collar pack, a flange at the coil end and four tie rods, allow to pre-load the coil heads up to 8 tons.

\subsection{Instrumentation and Protection}

The following instrumentation, needed to monitor assembly and testing is implemented in all models:
- Two spot heaters, to trigger transitions for quench studies, are placed one in the inner and one in the outer layer between the cable and the innermost end spacer, made of $50 \mu \mathrm{m}$ thick stainless steel foil glued between two $25 \mu \mathrm{m}$ thick polyimide foils.

- Voltage taps, in total 50 per model, for quench detection and location.

- Special collars with strain gauges near the poles to monitor the coil pre-stress in the inner and outer layers during magnet assembly, cool down and power testing. New capacitive pressure transducers were developed and already used at ambient and LN2 temperatures for collaring tests and coil modulus tests.

- End cage tie rods with strain gauges.

- Bullet gauges to monitor the force exerted by the coils against the thick end plates of the magnet.

- Gap opening transducers to monitor the status of the vertical yoke gap.

The mechanical instrumentation is calibrated both at ambient temperature and at $1.9 \mathrm{~K}$.

All models have quench heater strips placed between the outer layer and the ground insulation, to rapidly heat up the conductors in case of a quench. The calculated hot spot temperature assuming full energy dissipation in the coils and triggering of the heaters is around $240 \mathrm{~K}$.

\subsection{Fabrication and description of variants}

Coil winding of MBSMS1 started in June 1995 and since then seven models have been manufactured and some reworked (see Table 2). The cable insulation is all in polyimide and composed typically of two layers of $25 \mu \mathrm{m}$ thick tapes each overlapped by $48 \%$, and a third $70 \mu \mathrm{m}$ thick adhesive-coated layer, spaced by $2 \mathrm{~mm}$ to provide channels for helium penetration inside the coils. After winding, each layer is heated in a mould to $185^{\circ} \mathrm{C}$ for 30 minutes gluing turns firmly together. The coil heads are then impregnated with a heavily charged resin and the layers assembled with a grooved GII sheet placed in-between them. The inside cable ends are joined with an AgSn alloy, reinsulated and glued back onto the coil blocks in an operation referred to as reconditioning. The size and modulus of each layer are then measured to define pole and coil head shimming for collaring. Different collaring variants have been tried. Typically the coils are compressed to about 120-130 $\mathrm{MPa}$, the collaring rods are inserted and the external pressure released. The residual coil pre-stress is about 50-60 MPa on both layers. The end cages have been tightened generally after collaring. For models 4 and 5 the coils have been stretched during collaring with an internal mandrel reacting against the innermost coil end spacers and the cages pre-tightened at an intermediate stage to better distribute the longitudinal stresses inside the coil heads. An example of such a collaring is shown in Fig. 3. 


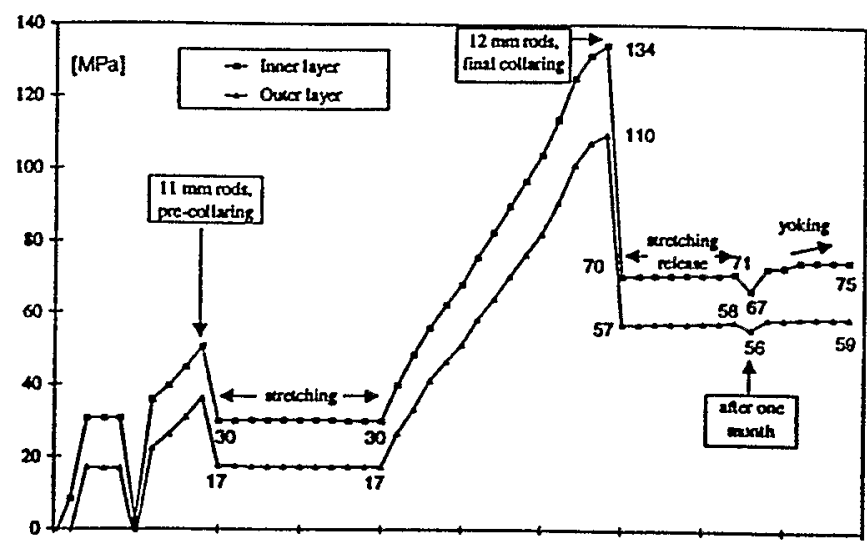

Figure 3. Collaring procedure for magnet MBSMS5

The main fabrication variants are shown in Table 2.

\begin{tabular}{|c|c|c|}
\hline \multicolumn{3}{|c|}{ Table 2:fabrication variants from MBSMS1 to MBSMS8 } \\
\hline magnet & coils* & assembly \\
\hline MBSMSI.VI & $\begin{array}{l}\text { non coated cable } \\
\text { type I end spacers }\end{array}$ & $\begin{array}{l}\text { reconditioning @ } 185^{\circ} \mathrm{C} \\
\text { end cage tightened }\end{array}$ \\
\hline MBSMS2.VI & $\begin{array}{l}\text { non coaled cable } \\
\text { type II end spacers }\end{array}$ & $\begin{array}{l}\text { reconditioning @ } 100^{\circ} \mathrm{C} \\
\text { end cage tightened }\end{array}$ \\
\hline MBSMS2.V2 & & end cage untightened \\
\hline MBSMS3.VI & $\begin{array}{l}\text { non coated cable } \\
\text { lype II \& Ila end spacers }\end{array}$ & $\begin{array}{l}\text { reconditioning under pressure } \\
\text { end cage tightened }\end{array}$ \\
\hline MBSMS1.V2 & & reconditioning under pressure \\
\hline MBSMS4.V1 & $\begin{array}{l}\text { in coated strands cable } \\
\text { type II end spacers }\end{array}$ & $\begin{array}{l}\text { reconditioning under pressure } \\
\text { end cage tightened } \\
\text { coil stretching }\end{array}$ \\
\hline MBSMS5.VI & $\begin{array}{l}\text { tin coated strands cable } \\
\text { type II end spacers } \\
\text { no intertum spacers } \\
150 \text { mm layer jump }\end{array}$ & $\begin{array}{l}\text { reconditioning under pressure } \\
\text { end cage tightened } \\
\text { collaring under stretching }\end{array}$ \\
\hline MBSMS6.VI & $\begin{array}{l}\text { tin coated strands cable } \\
\text { type Il end spacers } \\
\text { no interum spacers } \\
150 \text { mm layer jump } \\
\end{array}$ & $\begin{array}{l}\text { reconditioning under pressure } \\
\text { end cage tightened } \\
\text { different collar material }\end{array}$ \\
\hline MBSMS7.V1 & $\begin{array}{l}\text { tin coated strands cable } \\
\text { type III end spacers } \\
150 \mathrm{~mm} \text { layer jump } \\
\text { coil end not impregnated }\end{array}$ & $\begin{array}{l}\text { reconditioning under pressure } \\
\text { end cage not present }\end{array}$ \\
\hline MBSMS8.VI & $\begin{array}{l}\text { tin coated strands cable } \\
\text { type II end spacers } \\
\text { no interturn spacers } \\
150 \mathrm{~mm} \text { layer jump } \\
\text { different cable insulation }\end{array}$ & $\begin{array}{l}\text { reconditioning under pressure } \\
\text { end cage tightened }\end{array}$ \\
\hline $\begin{array}{l}\text { "end spacers ty } \\
\text { type I : mini } \\
\text { type Ila : isope }\end{array}$ & $\begin{array}{l}\text { design principle and materia } \\
\text { ideformation energy. GII: } \\
\text { tric, PEI: type III : Fe }\end{array}$ & $\begin{array}{l}\text { I: } \\
\text { type II : isoperimetric, GII: } \\
\text { ermilab design with "shoes", GI I; }\end{array}$ \\
\hline
\end{tabular}

\section{TEST RESULTS}

Cold tests have been made so far up to model 4 . Power test results are reported in Ref. [5]. All models are cooled directly to $1.9 \mathrm{~K}$, first training quenches were at $8.2,8.65,8.87$, and $8.67 \mathrm{~T}$ for models $1,2,3$, and 4 respectively, followed by slow training, occurring mainly in the first turn and in the transition between straight part and heads of the inner layer. Quenching with full energy deposition gives a hot spot temperature of $240 \mathrm{~K}$ and shows a safe magnet protection scheme. After a few such quenches, training switches to the first turn of the outer layer at a lowered unstable field level which remained however above $8.9 \mathrm{~T}$ for model 3. After a thermal cycle, this model retrained at $9.05 \mathrm{~T}$, showing limited training memory, but no longer exhibited the unstable behaviour mentioned above and reached $9.5 \mathrm{~T}$ after 16 training quenches.

\section{CONCLUSIONS}

A full test and result analysis program is going on for these models. In parallel the fabrication of new models and relevant variants is underway. Results show that general improvements in the assembly have brought better training behaviour, model 3 reaching $92 \%$ of the calculated short sample limit on its first quench, proving the validity of the basic design. So far there are no clear indications of a significant advantage coming from a particular end spacer type or from a specific technique like end cage tightening or coil stretching. Known weak spots have been addressed in later models: e.g. increased length of layer jump region, suppression of interturn spacers in the coil ends, smoother transitions between straight part and coil ends. Important aspects of dipole performance like dynamic behaviour and field quality are also addressed by the model program and will steer specific design solutions and fabrication techniques.

\section{ACKNOWLEDGEMENTS}

The authors wish to thank L. Evans, J. P. Gourber, R. Perin and P. Sievers for their constant support, the teams responsible of model assembly and measurements for their excellent work, R. Bossert and J. S. Brandt from FNAL for the design and supply of the end spacers for model 7 and industry for the supply of material and components.

\section{REFERENCES}

[1] The LHC Study Group. "The Large Hadron Collider Conceptual Design", CERN/AC/95-05 (LHC)

[2] R. Perin, "Status of LHC Programme and Magnet Development", IEEE Trans. Appl. Sup., Vol. 5(2) (1995) p.189

[3] L. Walckiers et al., "Power Tests of Single and Twin Aperture Superconducting Dipole Models for LHC", Proc. of MT14, 11-16 June 1995, Tampere, Finland

[4] D. Perini et al., "Structural Optimization of the Superconducting Aperture Dipole used as Coil Test Facility", CERN/AT-MA 95-129, August 1995

[5] A. Siemko et al., "Power Test Results of the First LHC Second Generation Superconducting Single Aperture 1m Long Dipole Models", ICEC, Kitakyshu, Japan, 20-24 May 1996. 


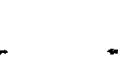




\title{
- LOW TEMPERATURE QUENCH PERFORMANCE OF FERMILAB LOW- $\beta$ INSERTION QUADRUPOLES
}

\author{
R. Bossert, S. Feher, S.A. Gourlay, T. Heger, J. Kerby, M.J. Lamm, P.J. Limon, P.O. Mazur, \\ T. Nicol, F. Nobrega, D. Orris, J.P. Ozelis, T. Peterson, P. Schlabach, J. Strait J. Tompkins, \\ A. Zlobin, Fermilab, Batavia, Illinois, USA; A. Lietzke, A.D. McInturff, R. Scanlan, \\ Lawrence Berkeley National Laboratory, Berkeley, California, USA
}

\section{Abstract}

The recently revived superconducting magnet program at Fermilab is currently focused on the development of high gradient quadrupoles for possible use in the Large Hadron Collider (LHC) interaction regions at CERN. In order to provide input for the new quadrupole design which will operate in superfluid helium, we have tested a Fermilab Tevatron low- $\beta$ quadrupole cold mass and compared its low temperature performance to a newly assembled heavily instrumented version which was mechanically modified to take advantage of the gain in critical current.

\section{INTRODUCTION}

Fermilab has successfully operated low- $\beta$ quadrupoles for the Tevatron $\mathrm{DO} / \mathrm{B} 0$ interaction regions at an operating gradient of $141 \mathrm{~T} / \mathrm{m}$ at $4.5 \mathrm{~K}$. Future low- $\beta$ insertions, in particular, the one proposed for the LHC[1], require a $50 \%$ higher operating field gradient. Fermilab in cooperation with Brookhaven National Laboratory (BNL) and Lawrence Berkeley National Laboratory (LBNL), is designing a higher gradient quadrupole suitable for the LHC low- $\beta$ insertions which uses NbTi conductor in superfluid helium[2]. A first step in this program has been to evaluate the feasibility of using a mechanically modified version of the existing Fermilab low- $\beta$ quadrupole for superfluid operation. We report on results from tests of two Fermilab-style superconducting high gradient quadrupoles.

\section{MAGNET DESCRIPTION}

The magnets for this study are $1.4 \mathrm{~m}$ long Tevatron low- $\beta$ quadrupoles. Details of the design have been described elsewhere[3,4]. This cold iron superconducting quadrupole has a 2 -shell, $\cos 2 \theta$ coil with a $76 \mathrm{~mm}$ aperture and an outer cold mass diameter of $276 \mathrm{~mm}$. The inner and outer coils are made from 36 strand Rutherford cable. The strands are $0.528 \mathrm{~mm}$ in diameter and contain $13 \mu \mathrm{m}$ filaments. There is a copper wedge in the inner coil whose primary purpose is to minimize the geometric 12- and 20-pole harmonics. Four inner to outer coil splices are located in the magnet lead end radially beyond the outer coil and are made through pre-formed solder- filled cable originating from the lead end pole turn. The coils are supported in the body by aluminum collars. The splice and the coil lead and return ends are clamped with a 4 piece G-10 collet assembly enclosed in a tapered cylindrical can. Iron yoke laminations surround the coil in the body region, and stainless steel laminations surround the end region cylindrical can. A welded stainless steel skin surrounds the yoke.

The two magnets differ in mechanical support and in instrumentation. One magnet (LBQ5425) was built as a spare for the Tevatron and as such has the nominal construction features and instrumentation for a production magnet. There are voltage taps across each quadrant (inner-outer coil pair). The aforementioned cylindrical end cans are made of stainless steel.

A finite element analysis mechanical model of the nominal production magnet at $1.8 \mathrm{~K}$ and full current excitation predicted inadequate coil support for both the body and the end regions[5]. Thus a second magnet (R54001) was built with the same tooling but with enhanced mechanical support. To increase the magnet end prestress at low temperature, the stainless steel end cans were replaced with aluminum cans. Kapton pole shims were inserted to increase the coil azimuthal prestress. These shims also increase the effective collar diameter to assure that there was an interference fit between the iron and collared coil. R54001 had several voltage taps in the inner coil concentrated near the pole turn and the copper wedge. Strain gauge transducers in the collars monitored changes in coil stress during manufacturing, cool down and excitation.

\section{MAGNET TESTS}

LBQ5425 was tested at the Lawrence Berkeley National Laboratory Magnet Test Facility in a horizontally oriented liquid helium dewar. The facility is designed to support superfluid helium at $1.8 \mathrm{~K}$ and at 1 atmosphere. R54001 was tested at the Fermilab Technical Support Section horizontal magnet test facility[6]. The test stand was originally designed to test SSC dipole cryostated cold masses at 4 atmospheres and temperatures from $4.6 \mathrm{~K}$ to $1.8 \mathrm{~K}$. The outer diameter of our low- $\beta$ quadrupole is roughly the same as an SSC dipole, thus we were able to build a special shorter length SSC-style cryostat to accommodate this magnet. 


\subsection{Magnet Training}

Both magnets were first trained at $4.3 \mathrm{~K}$ prior to superfluid testing. This allows one to compare the training of these magnets with previous low- $\beta$ quadrupoles[7] and to observe the change in magnet training between normal and superfluid helium. The training histories for LBQ5425 and R54001 are presented in Figures 1 and 2.

At $4.3 \mathrm{~K} \mathrm{LBQ5425}$ achieved $4700 \mathrm{~A}$ on the first quench and required 4 quenches to reach its plateau of $5150 \mathrm{~A}$. The magnet was warmed to room temperature and then cooled to $1.8 \mathrm{~K}$. The first quench was above $6000 \mathrm{~A}$, significantly higher than the $4.3 \mathrm{~K}$ plateau quenches. After two more training quenches, the quench current fell to near $5000 \mathrm{~A}$ and did not increase in the next 3 quenches. Two quenches (not shown) at $3.8 \mathrm{~K}$

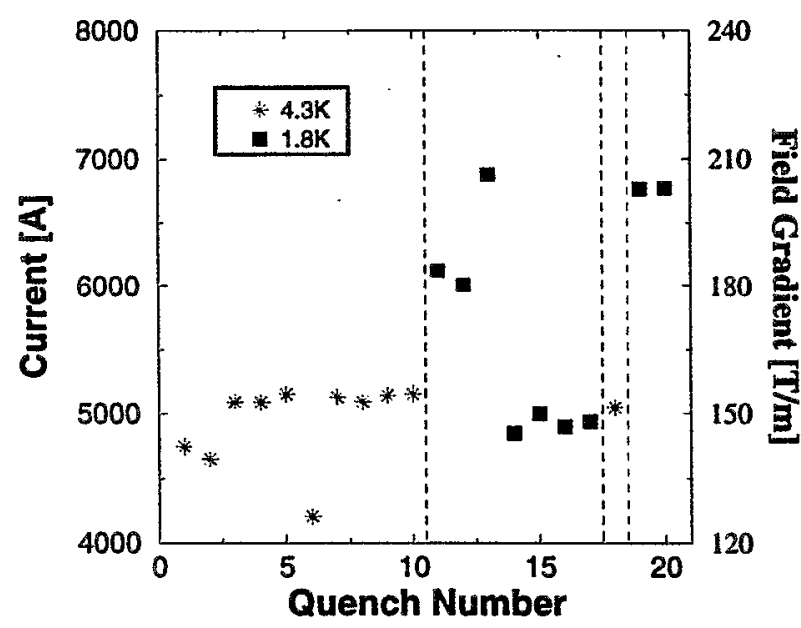

Figure 1: Quench training history for LBQ5425.

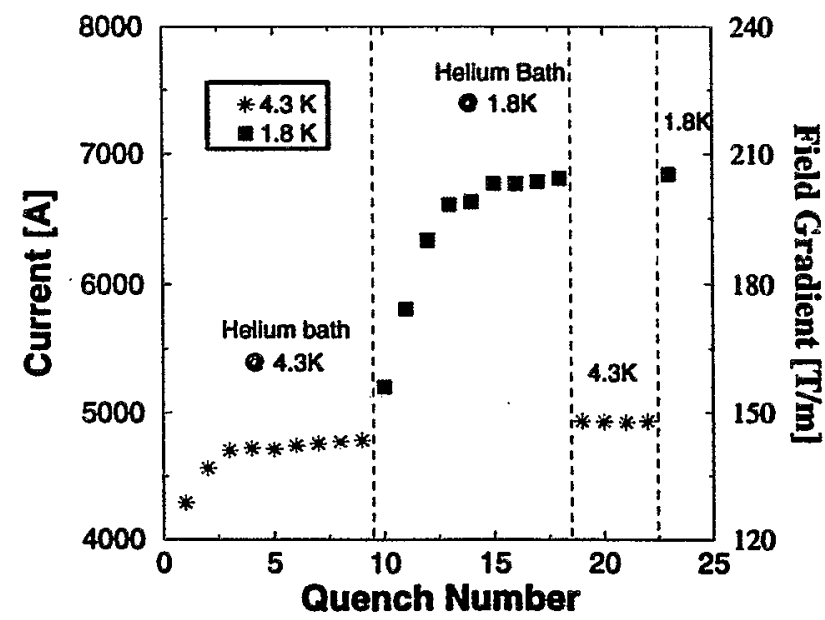

Figure 2: Quench training history for R54001. yielded similar results. The magnet was then warmed to $4.3 \mathrm{~K}$ and quenched again at just above $5000 \mathrm{~A}$. Finally, it was again cooled to $1.8 \mathrm{~K}$ and came within 100 amps of reaching its previous quench current maximum. The $5000 \mathrm{~A}$ quenches at $1.8 \mathrm{~K}$, as well as the low quench at $4.3 \mathrm{~K}$ (\#5 in Fig. 1), are likely due to insufficient coil support.

R54001, with enhanced mechanical structure, exhibited improved quench performance relative to LBQ5425, as seen in Fig. 2. At $4.3 \mathrm{~K}$ it came within $200 \mathrm{amps}$ of its quench plateau in three quenches. The training quenches largely occurred within the pole turn return end. After 9 quenches, but before it reached its plateau, the magnet was cooled to $1.8 \mathrm{~K}$. Here it exhibited significant training, but with monotonically increasing quench current. The training quenches occurred predominantly in the coil ends. After the $1.8 \mathrm{~K}$ testing the $4.3 \mathrm{~K}$ quench plateau was achieved. The plateau quenches occurred near the inner-outer coil splice.

\subsection{Temperature Dependence of Quench Current}

After 9 quenches at $1.8 \mathrm{~K}, \mathrm{R} 54001$ was quenched at several temperatures between $1.8 \mathrm{~K}$ and $4.3 \mathrm{~K}$ as shown in Fig. 3. There was a monotonic decrease in quench current with increasing temperature as expected for a magnet which is not limited by mechanical instabilities. However, the shape of the quench current vs. temperature, particularly near the $\lambda$-point, is not as predicted by temperature dependence of the conductor critical current. The deviation of the observed temperature dependence from the theoretical prediction can be explained by resistive heating in the coil, which increases as the current rises. The change in the curve shape at temperatures less than $2.17 \mathrm{~K}$ is likely due to the improvement of the coil cooling condition in superfluid helium.

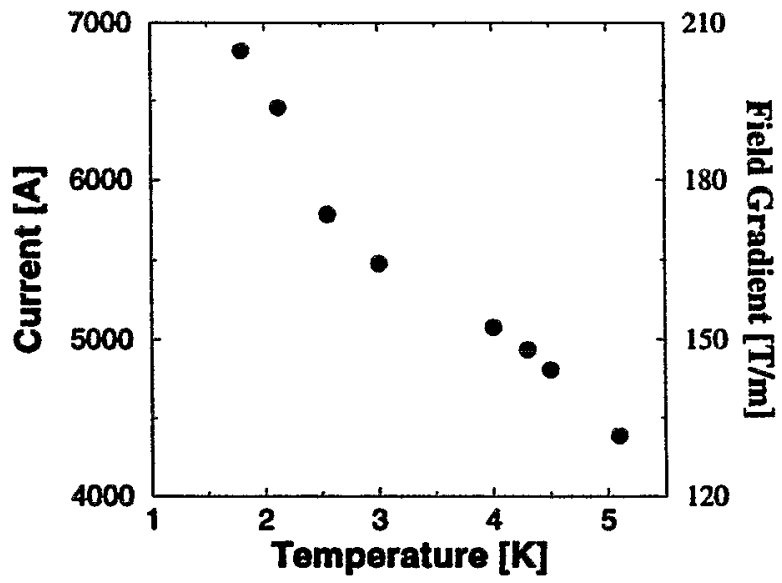

Figure 3: Quench current as a function of magnet temperature (nominal ramp rate of $16 \mathrm{~A} / \mathrm{s}$ ). 


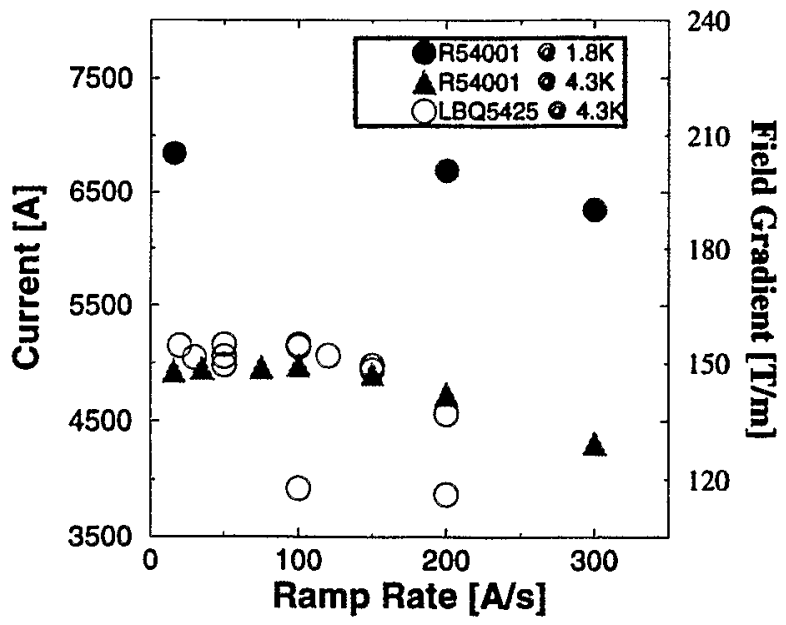

Figure 4: Quench current as a function of ramp rate. (Data were not taken at $1.8 \mathrm{~K}$ for LBQ5425.)

\subsection{Ramp Rate Dependence of Quench Current}

Figure 4 shows the dependence of the magnet quench current vs. the ramp rate for LBQ5425 and R54001. For both magnets, the quench current does not decrease with increased ramp rate up to $150 \mathrm{~A} / \mathrm{s}$. At higher ramp rates, cable heating due to AC losses decreases quench current. As above, we observed erratic quench behavior with LBQ5425 during these ramp rate tests.

Figure 5 shows the quench current normalized to the $16 \mathrm{~A} / \mathrm{s}$ value vs. ramp rate for $\mathrm{R} 54001$ measured at $4.3 \mathrm{~K}$ in normal helium and at $1.8 \mathrm{~K}$ in superfluid helium. An improvement in the quench current at high ramp rate in superfluid helium is evident due to improved coil cooling conditions.

\section{CONCLUSION}

Two Fermilab low- $\beta$ quadrupoles have been tested in superfluid helium. LBQ5425, a production spare for the Tevatron, reached $200 \mathrm{~T} / \mathrm{m}$ gradient at $1.8 \mathrm{~K}$ but exhibited erratic quench behavior. R54001, a magnet from the same design but with improved coil mechanical support, also reached $200 \mathrm{~T} / \mathrm{m}$ at $1.8 \mathrm{~K}$ and had significantly better quench behavior. Most quenches in R54001 above $1.9 \mathrm{~K}$ were generated by resistive coil heating. We also observed a significant improvement in the coil cooling condition and quench performance of this magnet in superfluid helium.

\section{ACKNOWLEDGMENTS}

We would like to thank the staff of the Fermilab and LBNL magnet test facilities for their assistance in these studies. Fermilab is operated by Universities Research

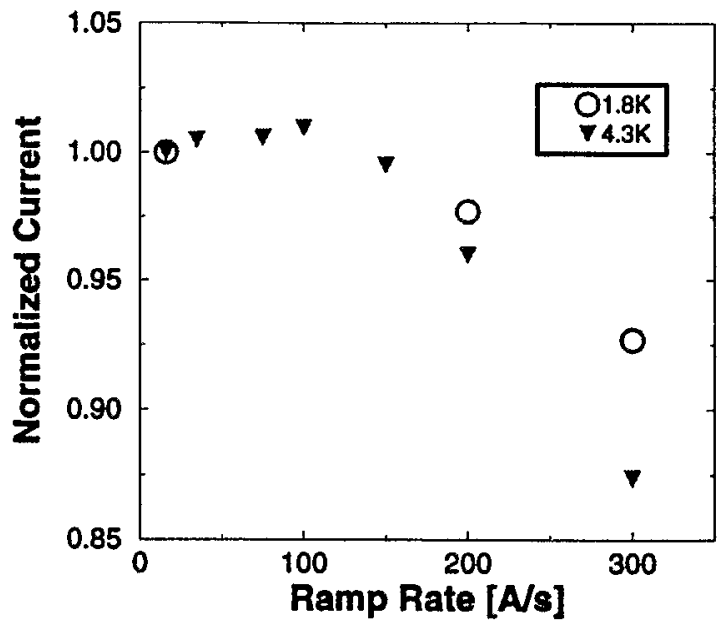

Figure 5: Magnet quench current normalized to the $16 \mathrm{~A} / \mathrm{s}$ value vs. current ramp rate.

Association Inc., under contract with the U.S. Department of Energy. The work at LBNL is supported by the Director, Office of Energy Research, Office of High Energy and Nuclear Physics, High Energy Physics Division, U.S. Department of Energy, under contract No. DE-AD03-76SF00098.

\section{REFERENCES}

[1] Large Hadron Collider Conceptual Design, CERN/AC/95-05 (LHC) 1995.

[2] R.C. Gupta et al, "Design of a High Gradient Quadrupole for the LHC Interaction Regions," this conference.

[3] A. D. McInturff et al. "The Fermilab Collider D0 Low- $\beta$ System," EPAC. Rome, Italy, Vol. 2, p 1264, June 1988.

[4] "D0 Low Beta Quadrupole Requirements and Specifications \#10," Fermilab Technical Support Section Internal Document February 15, 1990.

[5] T. Heger et al. "Summary of Finite Element Analysis of LBQ Magnets," Fermilab Technical Support Section Internal Note TS-96-009.

[6] J. Strait et al., "Fermilab R\&D Test Facility for SSC Magnets," Supercollider 1, p. 611989.

[7] S. Gourlay et al. "Quench Performance of Superconducting Quadrupole Magnets for the New Fermilab Low Beta Insertion," IEEE PAC Vol. 4, p. 2227, 1991. 



\title{
FIELD QUALITY OF THE MAIN DIPOLE MAGNETS FOR THE LHC ACCELERATOR
}

\author{
L. Bottura, A. Faus-Golfe, L. Walckiers, R. Wolf, CERN, Geneva, Switzerland
}

\section{ABSTRACT}

Short and long dipole model magnets for the LHC are measured in detail to optimize the field quality to the different operation phases of the accelerator. We will report on recent progress to understand and quantify effects related to the magnetization of the superconductor, to time dependent effects at fields corresponding to beam injection and to behaviour during acceleration. A parametrization for the measurements is proposed. The contributions from any misalignment of the dipoles and correctors magnets is compared to expected field quality of the dipoles.

\section{INTRODUCTION}

The LHC accelerator will produce head on collisions between beams of 7 TEV protons [1]. Beam optics calculations indicate that the performance of the LHC will be limited by the field quality mainly at low energy through a limitation of the dynamic aperture. Experience from the Tevatron [2] and Hera [3] machines have shown that time dependent effects on the field errors can lead to unstable beams during the injection and at the beginning of the acceleration where an abrupt "snap-back" to the field errors at start of injection takes place. Field ramps necessary for the beam acceleration create coupling currents between the strands of the superconducting cables of the winding, resulting in degradation of the field quality.

The field expansion used is relative to the main field, $B_{1}$, of the magnet at $x=R_{\text {wer }}=10 \mathrm{~mm}$. Here $n=1$ is a dipole field, $n=2$ is a quadrupole field etc. The $b_{\text {, and }}$ $a_{a}$ represent the normal and skew relative field errors.

$$
\mathrm{B}_{y}+i \mathrm{~B}_{x}=\sum_{n=1} C_{n}\left(\frac{z}{R_{n d}}\right)^{n-1}=B_{1} \sum_{n=1}^{-} \frac{\left(b_{n}+i a_{n}\right)}{10^{4}}\left(\frac{z}{R_{n d}}\right)^{n-1}
$$

This paper will concentrate on field errors that could dominate the behaviour of the LHC at low energy.

\section{ALIGNMENT OF THE DIPOLES AND THE END CORRECTORS}

The harmonics allowed by the symmetry of the dipole are large at injection field due to the cable magnetization. A misalignment of the dipole containing a multipole of order $n+1$ produces an additional one of order $\mathrm{n}$. Table 1 compares the errors generated by a misalignment of $0.5 \mathrm{~mm}$ to the expected random errors $\sigma\left(b_{n}\right)$ and $\sigma\left(a_{n}\right)$ of the dipole field. Moreover, the decapole and sextupole correctors mounted at each end of the main dipoles will produce similar quadrupole and octupole terms if misaligned with respect to the dipoles. Some care will therefore be needed to avoid systematic misalignment of the end correctors with respect to the main dipoles.

Table 1. Comparison for even harmonics (in units of $10^{-4}$ of injection field at $10 \mathrm{~mm}$ radius), between the errors resulting from a misalignment of $0.5 \mathrm{~mm}$ of the dipole and the standard deviation $(\sigma)$ of the field errors expected in the dipoles.

\begin{tabular}{|c|c|c|c|c|}
\hline $\mathrm{n}$ & $\begin{array}{c}\mathbf{b}_{\mathrm{n+1}} \text { expected } \\
\text { in the dipole }\end{array}$ & $\begin{array}{c}\sqrt{b_{1}^{3}+a_{c}^{2}} \\
0.5 \mathrm{~mm} \text { mis. }\end{array}$ & $\sigma\left(\mathrm{b}_{n}\right)$ & $\sigma(\mathrm{a})$ \\
\hline 2 & -3.9 & 0.4 & 0.4 & 1 \\
4 & 0.25 & 0.05 & 0.1 & 0.1 \\
6 & -0.026 & 0.008 & 0.06 & 0.01 \\
\hline
\end{tabular}

\section{INTERSTRAND EDDY CURRENTS}

Current ramps induce field distortions for all harmonics. These effects are dominated by coupling currents inversely proportional to the interstrands resistance of the superconducting cables [4]. Table 2 shows, for three different $10 \mathrm{~m}$ long models, that the deviations are important for all harmonics. Systematic field errors due to this effect could happen for instance due to the bending of the dipoles to the radius of curvature of the LHC.

A tight control of the LHC dynamic aperture is required. A systematic study based on these measured values was performed on the resulting loss of dynamic aperture at the beginning of the acceleration [5]. It makes the assumption that an exponential increase of the acceleration rate gives a constant perturbation all over the acceleration.

Table 2. Field errors due to cable coupling currents (in units of $10^{-1}$ of the injection dipole field at $10 \mathrm{~mm}$ ) at $8 \mathrm{~T} / 20 \mathrm{~min}$ ramp-rate, in four $10 \mathrm{~m}$ long dipole models. Also reported the average interstrand resistance as deduced from loss measurements.

\begin{tabular}{|c|c|c|c|c|}
\hline$n$ & MTP1A1 & MTP1A2 & MTPIA3 & MTPIN2 \\
\hline$b_{1}$ & 88 & 10.8 & & 9.8 \\
$b_{2}$ & -0.96 & -1.11 & -0.052 & 1.32 \\
$a_{2}$ & 10.8 & 0.89 & 1.052 & 1.06 \\
$b_{3}$ & 4.6 & 0.95 & 0.3 & 0.55 \\
$a_{3}$ & -0.16 & -0.19 & -0.035 & 0.14 \\
$b_{4}$ & 0.45 & -0.13 & 0.019 & -0.047 \\
$a_{4}$ & 0.82 & 0.08 & 0.13 & 0.24 \\
$b_{5}$ & 2.08 & 0.05 & 0.002 & -0.09 \\
$a_{5}$ & -0.34 & -0.02 & - & 0.026 \\
\hline$R_{c k s}(\mu \Omega)$ & 1.6 & 6.8 & 14 & 6.7 \\
\hline
\end{tabular}


This study shows that a spread comparable to the imperfections of MTPIAl would significantly reduce the LHC dynamic aperture during the ramp. What matters is really the spread of all harmonic terms for the whole population of dipoles, the average $b_{3}$ and $b_{3}$ can indeed be corrected with the end correctors. A spread comparable to the imperfections of MTP1A2 or even better of MTP1A3 would not degrade significantly the dynamic aperture. MTP1A2 might be seen as an acceptance limit at the expense of a significant increase of the already long ramp time. These conclusions should be confirmed by further tracking studies.

\section{MAGNETIZATION DECAY AND "SNAP-BACK"}

LHC superconducting dipoles are characterized by a significant drift of the magnetic field when the current is constant, with typical time scales in the order of several minutes to several hours [4]. At the restart of the ramp (beam acceleration phase) the field quickly bounces back, "snaps back", reaching within 25 to $40 \mathrm{~A}$ (approximately 20 to $30 \mathrm{mT}$ of field change) the original value at the start of the injection plateau. This can be of concern for the start of beam acceleration. All harmonics are subject to the field drift. On allowed harmonics the drift is systematic in the direction decreasing the contribution from the filaments magnetization, while it is random on non-allowed harmonics.

In accordance with previous measurements on Tevatron[2], HERA[3] and SSC[6] magnets, we have found that the amount of decay on all harmonics (and thus the snap-back) depends on the time spent at high current, and on the number of operating cycles. Typically, for the LHC dipoles, the decay requires very long flat-tops at high current to saturate (times longer than $1 / 2$ hour). A few cycles (of the order of 5) to nominal operating current produce an asymptotically stable situation. Quenching resets previous history effects. For machine operation this implies that magnets who have followed different histories will have a different behaviour at injection field.

We speculate that the long term drift and the snapback are associated with the interaction between the changing cable internal field associated with the strand current distribution and the magnetization of the single strands. We have started measurements of typical field decays and snap-back amplitudes, investigating on the influence of several parameters such as pre-cycles and temperature drifts in order to establish suitable procedures to minimize this effect. The main findings of these studies are listed below:
- Snap-back is associated with field change rather than with field change rate. This is shown in Fig. 1 where measurements in a $10 \mathrm{~m}$ long prototype are shown in the case of different ramp-rates after the injection platcau. The magnitude of the snap-back is approximately the same in all cases, but the time span for the snap-back scales inversely with the current ramp-rates.

- A pre-injection plateau at a lower current reduces the amount of field decay and snap-back at injection. A $\approx 25 \%$ smaller snap-back was measured on $b_{3}$ after a 15 minutes pre-injection plateau. The reduction is not a critical function of the pre-injection current.

- The magnitude of the snap-back on the allowed harmonics at different temperatures scales as the ratio of the contributions of $\mathrm{DC}$ magnetization to the harmonic at the same temperatures. This demonstrates the relation between field decay, snapback and DC magnetization.

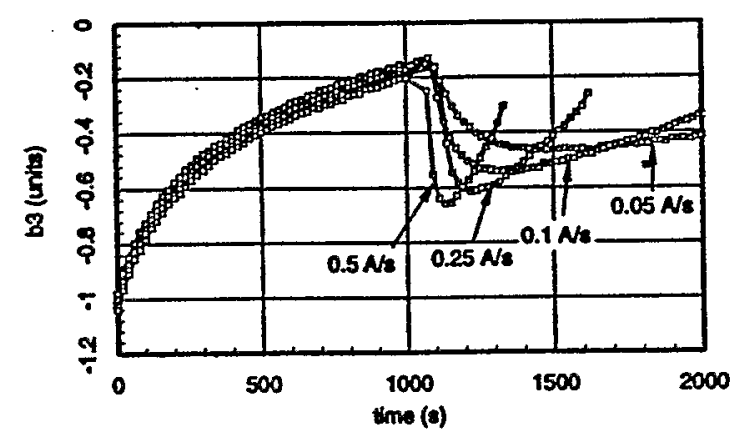

Figure 1. Snap-back in a $10 \mathrm{~m}$ long dipole prototype (MTPIN2) at different ramp-rates after the injection plateau.

In summary, a pre-injection plateau before the injection phase decreases the snap-back. The residual magnitude can be accommodated by slow ramps giving time for the corrector scheme to react. Typical magnitudes for the snap-back of the first harmonics in the LHC $10 \mathrm{~m}$ long prototypes, measured after several days of operation, are reported in Tab. 3. Systematic effects are expected only on the allowed harmonics.

Table 3. Change of multipoles due to snap-back for the LHC dipoles, estimated from the measured snap-back in $10 \mathrm{~m}$ long prototypes. All quantities in units of $10^{-4}$ of the main ficld, at $10 \mathrm{~mm}$ reference radius. The standard deviations expected from geometrical errors in the magnets series are quoted as a reference.

\begin{tabular}{|c|c|c|}
\hline component & $\Delta$ (Snap-Back) & $\sigma$ (geometric) \\
\hline$b_{2}$ & \pm 0.1 & 0.4 \\
$a_{2}$ & \pm 0.5 & 1 \\
$b_{3}$ & \pm 0.8 & 0.5 \\
$a_{3}$ & \pm 0.2 & 0.15 \\
$b_{4}$ & \pm 0.5 & 0.1 \\
$a_{4}$ & 0.0 & 0.1 \\
$b_{3}$ & -0.1 & 0.05 \\
$a_{3}$ & 0.0 & 0.04 \\
\hline
\end{tabular}




\section{PARAMETRIZATION OF THE MEASUREMENTS}

In a most general way, we can write that the field B produced by a magnet in arbitrary operating conditions is approximated by:

$$
B=B_{g e o m}+B_{s}+B_{\Delta x \Delta y}+B_{A C}+B_{M}+B_{\Delta K}
$$

where we have evidenced the various contribution of winding geometry $\mathbf{B}_{\text {seom }}$ (including the linear iron contribution), iron saturation $B_{s}$, winding movements $\mathbf{B}_{A_{A}, A_{1}}$ coupling current $\mathbf{B}_{A C}$ filaments magnetization $\mathbf{B}_{\boldsymbol{\mu}}$ and long term magnetization drift and snap-back $\mathbf{B}_{A_{w}}$ For each of them we are testing suitable parametric dependencies based on existing models or empirical fits.

The present scaling for the coefficients $C_{a}$ is given in Tab. 4 , where $C_{m}$ indicates the main field component.

Table 4. Scaling of the field contributions proposed for a parametric representation.

\begin{tabular}{|c|c|c|}
\hline & Main field & Field erros $(n \lambda n+1)$ \\
\hline $\mathbf{B}_{\text {reom }}$ & $C_{m}^{m-m}=\gamma_{m} I$ & $C_{*}{ }^{200 *}=\gamma_{n} C_{n}$ \\
\hline $\mathbf{B}+\mathbf{B}_{\Delta-A_{1}}$ & $C_{s}=\sigma_{\mu s} I^{2}+\sigma_{\mu s} l^{\prime \prime}+\sigma_{\mu} l^{2}$ & $C_{m}^{\mu}=\sigma_{m, 2} C_{m}^{2}+\sigma_{n, 3} C_{m}^{3}+\sigma_{k, 4} C_{m}^{A}$ \\
\hline $\mathbf{B}_{A C}$ & $C_{-}^{N C}=\tau_{-} \frac{d l}{d t}$ & $C_{n}^{A C}=\tau_{n} \frac{d C_{n}}{d t}$ \\
\hline $\mathbf{B}_{\mu}$ & $C_{=}^{\mu}=\mu_{-\infty}+\frac{\mu_{m, 1}}{l}$ & $c_{n}^{N}=\mu_{n, 0}+\frac{\mu_{n, 1}}{C_{m}}$ \\
\hline $\mathbf{B}_{\Delta \mu}$ & 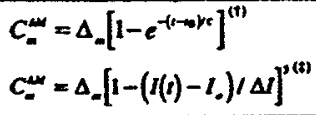 & $\begin{array}{l}C_{n}^{\Delta N}=\Delta_{n}\left[1-e^{-\left(1-1_{0}\right) / t}\right]^{(1)} \\
C_{n}^{\Delta N}=\Delta_{n}\left[1-\left(1()-I_{0}\right) / \Delta\right]^{(t)}\end{array}$ \\
\hline
\end{tabular}

(") During a $I=I_{0}$ current plateau starting at $t=t_{0}$

(") At ramp restart, between starting current $I_{0}$ and current $I_{0}+\Delta$

\section{IMPLICATION FOR THE SERIES MEASUREMENTS}

A coils assembly able to measure the integral of the field is under construction [7]. It is composed of 14 measuring lengths separated for mechanical reasons by gaps equal to the twist pitch of the cable. It will allow much faster measurements for the series. The errors avoided by an integrated measurement are listed below.

- The residual error from a measuring coils having in average the length of the twist pitch that varies slightly between cable manufacturers is larger than the standard deviation from magnet to magnet.

- Time dependent effects have a larger amplitude when approaching the ends of the magnets.

- Point like variations of eddy currents have been measured.

- The randomness of the snap back effects is not yet understood. We believe that the standard deviation measured along the $15 \mathrm{~m}$ of the magnets can be as high as the deviation between magnets.
The request to be able to measure the dipole axis and verify the centering of the spool piece correctors is not possible with a integral shaft. These measurements will be performed during warm tests of the final assemblies with the help of a short coil. Differences between the axis positions in warm and cold conditions will be verified on a reduced number of magnets.

\section{CONCLUSION}

Exploratory measurements of both short and long models of the LHC superconducting dipole magnets indicate the importance of methodically separating different effects. The reproduciblity of the time dependent effects and dynamic effects may dominate the low field quality with respect to superconductor magnetization and harmonics due to the conductors locations. We have defined a model for the magnetic measurements in order to quantify and eventually correct these effects that could limit the dynamic aperture of the LHC machine at low energy.

\section{REFERENCES}

[1] "The Large Hadron Collider, Accelerator Project", LHC Study Group, CERN/AC/95-05(LHC), Aug.1995.

[2] R.W. Hanft, et al., "Studies of Time Dependent Fields in Tevatron Superconducting Dipole Magnets", IEEE Trans. Magn., 25, (2), 1647-1651, 1989.

[3] H. Brueck, et al., "Time Dependent Field Distortions from Magnetization Currents in the Superconducting Hera Magnets", Cryogenics, 30, 605-609, 1990.

[4] J. Buckley, D. Richter, L. Walckiers, R. Wolf, "Dynamic magnetic measurements of superconducting magnets for the LHC", IEEE Trans. Of Appl. Sup., 5, (2), p 1024, 1995

[5] A. Faus-Golfe "Minimization of the ramp-induced non-linear field imperfections in LHC", LHC Project Note 9, May 1995.

[6] A. Devred, et al., "Time Decay Measurements of the Sextupole Component of the Magnetic Field in a 4-cm Aperture", 17-m-Long SSC Dipole Magnet Prototype, Proc. of $14^{\text {th }}$ Particle Accelerator Conference, San Francisco CA, May 6-9, 1991.

[7] P. Sievers, "Towards series measurements of LHC magnets",CERN AT/94-11(MA), June 1994. 


\title{
Dynamic Aperture Limitations of the LHC in Physic Conditions due to Low-Beta Insertions
}

\author{
A. Faus-Golfe and A. Verdier \\ CERN/SL
}

\begin{abstract}
The dynamic aperture of LHC in collision is determined by the multipole field errors of the low-beta triplet quadrupoles. Their effect, combined with that of the systematic and random magnetic imperfections expected in all machine magnets is analysed here. The effect of crossing angle is taken into account.
\end{abstract}

\section{INTRODUCTION}

The effects of the multipole components in the low- $\beta$ quadrupoles was investigated some time ago [1] on a preliminary version of LHC, with a short term tracking. It was noticed that the errors in the triplet dominate the non-linear dynamics. As the field errors are now better known, it is important to remake the analysis with long term tracking including synchrotron oscillations. This is the subject of this paper, it is shown that the triplet errors still dominate with the latest LHC design.

\section{THE LHC LATTICE.}

The LHC lattice we consider is that described in the conceptual design report [2]. It is labelled version 4.2 and has four physics insertions where $\beta^{*}$ has a value of $0.5 \mathrm{~m}$ in physics conditions in both planes, in order to achieve a luminosity of about $10^{84} \mathrm{~cm}^{-2} \mathrm{~s}^{-1}$. This low $\beta^{*}$ value is obtained by means of highly focusing quadrupoles triplets [2][3]. As the free space between the interaction point and the first low- $\beta$ quadrupole is about $20 \mathrm{~m}$, the peak $\beta$-value in the triplets is of the order of $4000 \mathrm{~m}$. This gives an enormous importance to the multipole components at this location as shown below.

\section{FIELD ERRORS IN THE LHC MAGNETS.}

The field errors are divided into:

- average or design errors, associated with a given magnet type. They obey symmetry rule, e.g. in the dipoles, all $b_{n}$ with $n$ even change sign when going from an inner aperture to an outer one.

- uncertainty on average errors. This is indicated by a \pm sign in the tables [2]. They are randomly assigned to each L.HC arc.
- random errors from magnet to magnet, distributed according to a Gaussian law cut at $3 \sigma$, except for the low- $\beta$ quadrupoles where the cut is at $2 \sigma$.

An input for the MAD program [4], used for the optics calculations and trajectory tracking, has been made to specify all errors at a time, so that the distribution of a given error remains the same whatever the others. This procedure is a little heavy but it makes it possible to obtain the effect of any multipole component in any context.

\subsection{Arc cell magnets.}

The multipole errors in the dipoles and the arc quadrupoles are those given in the "yellow book" [2]. It can be shown with equation 1 below that the quadrupole errors are only a few percents of the dipole errors and hence negligible, except $b_{B}$ which will be studied separately.

\subsection{Other LHC magnets.}

Quadrupoles similar to the arc cell quadrupoles are used to match the insertions and dispersion suppressors. They behave similarly to the arc cell quadrupoles.

The two LHC beams are brought in collision by means of dipole magnets sitting between the dispersion suppressors and the insertions. Under physics conditions the value of the $\beta$ functions in these dipoles is large. Their effect on the dynamic aperture is then important. As they have not yet been designed, no error table is available and they are not considered here.

In the cleaning, dump and RF insertions there are conventional quadrupoles. Their field errors are determined by the design and are known within one percent. Their effect is examined below.

\subsection{Low- $\beta$ quadrupoles.}

The multipole errors in the low- $\beta$ quadrupoles are given in table 1 [3]. In order to compare them with the field errors in the dipoles, an equivalent dipole error $b_{n, q, d}$ can be defined :

$$
b_{n, q, d}=b_{n, q} \frac{K r l_{q}}{\theta}
$$

where $K$ is the normalised quadrupole gradient $\left(0.00972 \mathrm{~m}^{-2}\right), l_{q}$ the quadrupole length $(5.5 \mathrm{~m}), \theta$ the angle of the dipole (5mrad) and $b_{n, q}$ the field error in the dipoles [2]. It can be noticed that the mean $b_{8}$ and $b_{10}$, which are the only important components, are smaller 
Table 1: Multipole errors in the low-beta quadrupoles in physics. The errors are field enrors at $10 \mathrm{~mm}$ from the quadrupole axis, in units of $10^{-4}$. b11, b12 and b13 are zero.

\begin{tabular}{|c|c|c|c|c|}
\hline & \multicolumn{2}{|c|}{ Mean } & \multicolumn{2}{c|}{ Random } \\
\hline$n$ & $b_{n}$ & $a_{n}$ & $b_{n}$ & $a_{n}$ \\
\hline 3 & 0 & 0 & 0.68 & 0.68 \\
4 & 0 & 0 & 0.23 & 0.23 \\
5 & 0 & 0 & 0.08 & 0.08 \\
6 & -0.008 & 0 & 0.028 & 0.028 \\
7 & 0 & 0 & 0.004 & 0.004 \\
8 & 0 & 0 & 0.0009 & 0.0009 \\
9 & 0 & 0 & 0.0002 & 0.0002 \\
10 & -0.005 & 0 & 0.00005 & 0.00005 \\
\hline
\end{tabular}

than those in the main dipoles by one order of magnitude. However, in order to compare their effects, the non-linear aberrations, which scale with $K r l_{q} \beta^{\frac{\pi}{3}}$ for $b_{n}$ have to be compared.

For the low- $\beta$ quadrupoles, $\beta_{x}$ is $4000 \mathrm{~m}$ in half of them and 1000 in the others with opposite sign. The value of $b_{8} K r l_{q} \beta_{x}{ }^{8}$ merely summel over all triplet quadrupoles is $4.3 \times 10^{6} \mathrm{~m}^{3}$. For the dipoles the similar quantity $b_{8} \theta \beta_{x}{ }^{3}$ is only $4.1 \times 10^{4} \mathrm{~m}^{3}$. Such a calculation is extremely crude but it shows that the effect of the quadrupoles on the non-linear betatron oscillations is expected to be larger than that of the dipoles by about two orders of magnitude.

The accurate estimation of the effect of these errors is done below by long term tracking of particle trajectories.

\section{DYNAMIC APERTURE.}

The dynamic aperture is defined as the maximum action in both planes simultaneously for which transverse betatron oscillations remain stable over $10^{5}$ turns. Tracking the particle trajectories is done with the MAD program [4]. The trajectories start at the interaction point IP1 where both $\beta^{*}$ are $0.5 \mathrm{~m}$. Both starting coordinates are equal, the initial relative momentum deviation is $3.6 \times 10^{-4}$ and all canonical momenta are zero. Under these conditions, the initial amplitude (the same value in $\mathrm{x}$ and $\mathrm{y}$ ) has to be multiplied by $\sqrt{2}$ in order to give the maximum radius in the $\{x, y\}$ plane.

The tunes are $Q x=63.28, Q_{y}=63.31, Q s=0.001(16 M V$ $R F$ voltage for an energy of $T \mathrm{TeV}$ ).

Neither alignment errors nor quadrupole errors are included $(a 2=b 2=0)$. The $b 3$ and $b 5$ compensators, the so called "spool pieces" in the dipole ends, are turned on. Both tune derivatives are set to 1.0 after the errors are introduced in order to take into account the tune modulation associated with the synchrotron oscillations. A physical aperture limitation has only been introduced only for the case of individual multipoles (section 7).

\section{DYNAMIC APERTURE DUE TO THE ARC CELL ERRORS.}

The dynamic apertures as well as their average and standard deviation obtained for 10 random distributions of the errors in the arc cell magnets are listed in table 2. Under physics

Table 2: Dynamic aperture associated with the dipole and arc quadrupole errors, both systematic and random.

\begin{tabular}{|c|c|c|c|c|}
\hline seed & \multicolumn{2}{|c|}{$10^{\frac{t}{\text { tums }}}$} & \multicolumn{2}{c|}{$10^{\mathrm{B}}$ turns } \\
\hline eopt & $\mathrm{x}$ at QF/mm & $\mathrm{n}_{\sigma}$ & $\mathrm{x}$ at $\mathrm{QF} / \mathrm{mm}$ & $\mathbf{n}_{\sigma}$ \\
\hline 1 & 11.9 & 39.2 & 9.6 & 31.7 \\
2 & 12.7 & 42.0 & 11.6 & 38.3 \\
3 & 11.9 & 39.2 & 11.3 & 37.3 \\
4 & 13.0 & 42.9 & 11.9 & 39.2 \\
5 & 10.7 & 35.5 & 9.1 & 29.9 \\
6 & 12.7 & 42.0 & 11.6 & 38.3 \\
7 & 11.9 & 39.2 & 11.0 & 36.4 \\
8 & 15.6 & 51.3 & 14.7 & 48.5 \\
9 & 13.9 & 45.7 & 11.3 & 37.3 \\
10 & 11.0 & 36.4 & 10.7 & 35.5 \\
\hline $\mathrm{x}_{\mathrm{av}}$ & 12.5 & 41.4 & 11.3 & 37.3 \\
$\sigma_{\boldsymbol{s}}$ & 1.3 & 4.4 & 1.4 & 4.7 \\
\hline
\end{tabular}

conditions the primary collimators limit the aperture to $6 \sigma$ at most and the betatron motion must be regular enough for the particles scattered by the primary collimators and reaching $10 \sigma$ [6]. This is largely fulfilled with a dynamic aperture of $10 \sigma$ at $10^{4}$ turns.

The results in table 2 show that we are well above these limits. If the systematic multipoles in the warm quadrupoles are added, the reduction of the dynamic aperture is of the order of $5 \%$, i.e. negligible.

\section{DYNAMIC APERTURE DUE TO THE LOW- $\beta$ QUADRUPOLES}

\subsection{No crossing angle}

Table 3: Average dynamic aperture associated with the dipole, arc quadrupole and low- $\beta$ quadrupole errors, both systematic and random.

\begin{tabular}{|c|c|c|c|c|}
\hline & \multicolumn{2}{|c|}{$10^{4}$ turns } & \multicolumn{2}{c|}{$10^{5} \mathrm{tums}$} \\
\hline & $\mathrm{x}$ at QF/mm & $\mathrm{n}_{\sigma}$ & $\mathrm{x}$ at $\mathrm{QF} / \mathrm{mm}$ & $\mathbf{n}_{\sigma}$ \\
\hline $\mathrm{x}_{a v}$ & 4.0 & 13.3 & 3.8 & 12.5 \\
$\sigma_{x}$ & 0.2 & 0.6 & 0.2 & 0.7 \\
\hline
\end{tabular}

The summary of the results of the computation of the dynamic apertures obtained for the same 10 random distributions of the errors as in the previous section is shown in table 3. Its variability with the distribution of the random errors is considerably reduced compared with that in table $2:$ It is clear that the multipoles in the low- $\beta$ quadrupoles dominate the dynamic aperture.

\subsection{Crossing angle}

The LHC beams cross at a full angle of $200 \mu \mathrm{rad}$. This is achieved with a local closed orbit distortion made independently in both rings. The angle of $100 \mu \mathrm{rad}$ in each ring makes a closed orbit excursion of about $5 \mathrm{~mm}$ in the $10 w-\beta$ quadrupoles. Because of the multipole components in the quadrupoles, lower order multipoles are created (this is often referred to as "multipole feed-down"). The summary of 
Table 4: Dynamic aperture associated with the dipole, arc quadrupole and low- $\beta$ quadrupole errors, both systematic and random. Crossing angle scheme on. If the warm quads are added, $x_{a r}$ increases by $3 \%$.

\begin{tabular}{|c|c|c|c|c|}
\hline & \multicolumn{2}{|c|}{$10^{\frac{s}{t} \text { turns }}$} & \multicolumn{2}{c|}{$10^{5}$ turns } \\
\hline & $\mathrm{x} \mathrm{at} \mathrm{QF/mm}$ & $\mathrm{n}_{\sigma}$ & $\mathrm{x}$ at QF/mm & $\mathrm{n}_{\sigma}$ \\
\hline $\mathrm{x}_{\boldsymbol{a v}}$ & 3.2 & 10.6 & 3.0 & 9.9 \\
$\sigma_{\boldsymbol{x}}$ & 0.2 & 0.6 & 0.2 & 0.6 \\
\hline
\end{tabular}

the results of the computation of the dynamic aperture obtained by turning the crossing angle scheme on, with the errors described in the preceding section, is shown in table 4. A reduction by a factor 0.8 is observed.

In fact, turning the crossing angle scheme on produces little effects on the linear optics. The tunes-shifts are $1.1 \times 10^{-4}$ in the horizontal and $-3.3 \times 10^{-3}$ in the vertical plane, they are compensated by means of auxiliary quadrupoles [7] which produce a negligible optics perturbation. The chromaticities do not change. A negligible linear coupling is due to the feed-down effect of the skew multipole components (the resulting width of the coupling resonance is $3.4 \times 10^{-3}$, it is not compensated). The observed effect must be then due to the "feed-down" of low-order multipoles. Indeed the anharmonicity increases by about $20 \%$ on average when the crossing angle is turned on.

\section{EFFECT OF INDIVIDUAL COMPONENTS IN THE LOW- $\beta$ QUADRUPOLES.}

The dynamic aperture was computed for each multipole component in the low- $\beta$ quadrupoles in presence of the chromaticity sextupoles only. The results are shown in table 5. If the aperture limitation due to the vacuum chamber is introduced both in the arc quadrupoles and in the triplet, the results are identical, with the present amplitude steps.

The dominating multipole is b10. This can be easily understood by computing the field errors at the maximum amplitude in the low- $\beta$ quadrupoles. For the case where the maximum horizontal amplitude is $3.4 \mathrm{~mm}$ in the arc where $\beta_{z}$ is $181 \mathrm{~m}$, it is $16 \mathrm{~mm}$ in the low- $\beta$ quadrupoles where $\beta_{x}$ is $4000 \mathrm{~m}$. The vertical field error at $16 \mathrm{~mm}$ is then $0.084 \times 10^{-4}$ for $b 6$ and $-0.34 \times 10^{-4}$ for b10. It is clear that the reference radius of $10 \mathrm{~mm}$ does not give a right idea of the relevant errors in the low- $\beta$ quadrupoles.

\section{SOLUTIONS TO THE MULTIPOLE PROBLEM.}

In order to obtain a dynamic aperture of $10 \sigma$, it is probably not possible to re-optimise the field error in the triplet, as a reduction of b10 by a factor of about 5 , which may not be possible, gives a just acceptable dynamic aperture (table 5). A safe solution is to use compensators for the most dangerous component and this is presently under study. With the present field errors, three b10 compensators placed close to
Table 5: Dynamic aperture associated with low- $\beta$ quadrupole systematic errors and chromaticity sextupoles only (upper), random b6 and b10 are added in the lower part (the r.m.s. deviation in indicated with \pm . Crossing angle scheme on $\hat{x}$ is the maximum amplitude at QF. The dynamic aperture according to the Liapounov exponent is given in Liap.

\begin{tabular}{|c|c|c|c|c|c|}
\hline & \multicolumn{2}{|c|}{$10^{4}$ turns } & \multicolumn{2}{c|}{$10^{5}$ turns } & \\
\hline mult. & $\hat{x} / \mathrm{mm}$ & $\mathrm{n}_{\sigma}$ & $\hat{x} / \mathrm{mm}$ & $\mathrm{n}_{\sigma}$ & Liap. \\
\hline $\mathrm{b} 6$ & 7.1 & 23.3 & 7.1 & 23.3 & 23.3 \\
$\mathrm{~b} 10$ & 3.4 & 11.2 & 3.4 & 11.2 & 11.2 \\
$\mathrm{~b} 6+\mathrm{b} 10$ & 3.7 & 12.1 & 3.4 & 11.2 & 11.2 \\
$\mathrm{~b} 6+.2 \mathrm{~b} 10$ & 4.8 & 15.9 & 4.5 & 14.9 & \\
\hline $\mathrm{b} 6+\mathrm{r}$ & $4.8 \pm 0.4$ & 15.9 & $4.3 \pm 0.3$ & 14.3 & \\
$\mathrm{~b} 10+\mathrm{r}$ & $3.4 \pm 0.0$ & 11.2 & $3.3 \pm 0.1$ & 11.0 & \\
$\mathrm{~b} 6+\mathrm{b} 10+\mathrm{r}$ & $3.4 \pm 0.1$ & 11.2 & $3.3 \pm 0.1$ & 10.7 & \\
\hline
\end{tabular}

each quadrupole are probably necessary.

Eventually increasing $\beta^{*}$ reduces the maximum $\beta^{\prime}$ 's in the low- $\beta$ quadrupoles. The dynamic aperture computed with a $\beta^{*}$ of $1 \mathrm{~m}$ in presence of $\mathrm{b} 6, \mathrm{~b} 10$ and chromaticity sextupoles increases to $28 \sigma$, which shows the its sensitivity to $\beta^{*}$.

\section{CONCLUSION.}

The multipole errors in the low- $\beta$ quadrupoles limit the dynamic aperture under physics conditions because of the large values of the $\beta$-functions in these quadrupoles. They are one of the main contributors to the lower limit on $\beta^{*}$. A compensation system, necessary to reach very small $\beta^{*}$ is now under study.

\section{REFERENCES}

[1] B.T. Leemann and W. Scandale, Performance limitations of LHC low beta insertions. EPAC Rome, June 1988.

[2] The LHC study group, The LARGE HADRON COLLIDER Conceptual design. CERN/AC/95-05(LHC), 20 October 1995.

[3] R. Ostojic, T.M. Taylor, private communication.

[4] H. Grote and F.C. Iselin, The MAD program (Methodical Accelerator Design) version 8.16, User's reference manual, CERN/SL/90-13(AP), (rev. 4) (March 27, 1995).

[5] M. Giesch, Twin aperture quadrupoles for the LHC cleaning insertions. Magnet Technology Conference (MT14), June 1995. Tampere, Finland. Also CERN AT/95-15(MA) and LHC Note 323.

[6] J. B. Jeanneret, private communication.

[7] A. Verdier, Operational Q-shifts and 62 compensation in LHC. LHC Project Note 26 (January 8, 1996). 



\title{
- FIELD QUALITY IN SUPERCONDUCTING MAGNETS FOR LARGE PARTICLE ACCELERATORS *
}

\author{
Ramesh Gupta, RHIC Project, Brookhaven National Laboratory, Upton, NY 11973, USA
}

\section{Abstract}

The expected field quality in superconducting magnets for large particle accelerators has improved over a period of time due to the development and application of a number of techniques. These design techniques will be described and as an example the expected harmonics in an improved design of the $50 \mathrm{~mm}$ aperture dipole magnets for the Superconducting Super Collider (SSC) will be presented. This field quality is based on experience with the Relativistic Heavy Ion Collider (RHIC) and SSC magnet programs. The initial results of a design approach will also be presented where the first design itself is adopted to produce good field quality in RHIC D0 insertion magnet.

\section{INTRODUCTION}

The purpose of this paper is (a) to give a brief outline of the concepts which either have been used or can be used in designing and then assuring good field quality in accelerator magnets, $(b)$ to present a design concept which avoids the need of a normal magnetic design iteration which is both time consuming and expensive, $(c)$ to present methods which can be used to control and match the field quality in the magnets built by several vendors, $(d)$ to present techniques which overcome the influence of normal errors in parts and assembly and $(e)$ to apply these concepts in a quantitative way to estimate field quality in a magnet which can be used as a reference for the next generation machines.

The field quality in accelerator magnets is characterized in terms of the normal and skew harmonics, $b_{n}$ and $a_{n}$. They are defined in the following expression

$$
B_{y}+i B_{x}=10^{-4} \times B_{R 0} \sum_{n=0}^{\infty}\left(b_{n}+i a_{n}\right)[(x+i y) / R]^{n},
$$

where $B_{\mathrm{x}}$ and $B_{\mathrm{y}}$ are the components of field at $(x, y)$ and $B_{\mathrm{R} 0}$ is the magnitude of the field due to fundamental harmonic at a "reference radius" $R$.

\section{METHODS FOR CONTROLLING FIELD QUALITY}

The methods for controlling field quality are only discussed briefly here (see references [1] to [9] for 'details).

*Work supported by the U.S. Department of Energy under contract No. DE-AC02-76CH00016.

\subsection{Allowed Harmonics and Pre-stress on Coils}

Two allowed harmonics can be adjusted by adjusting (a) the coil-to-midplane gaps (or midplane shims) and (b) the coil pole shims. This is an efficient technique for small adjustments in lower order harmonics (e.g., $b_{2}$ and $b_{4}$ in dipoles). It may be used either for normal crosssection iteration or to compensate for the differences in field quality of the magnets built by different vendors based on the same magnetic design.

The pre-stress on the collared coil, however, will change if there is a net change in the combined thickness of the pole and midplane shims. A small variation in pre-stress may be tolerated but if it is larger than a few kpsi, then to avoid it one should adjust the coil curing pressures to change the cured coil size. The first magnet of a series usually requires larger adjustment in field harmonics. This is further complicated by the experience that the desired pre-stress on the coils may also not be obtained by the nominal size pole shims. A change in pole shims in order to get the desired prestress also changes the field quality.

To deal with the above difficulties, an approach has been developed for the RHIC $100 \mathrm{~mm}$ aperture insertion dipole D0 [5] with the goal that the first design itself can be adopted for production by applying small adjustments which are part of the initial design. A third parameter to provide the desired pre-stress on the coils is obtained by increasing the effective thickness of one (or more) selected wedge(s) by changing the number of layers of insulation on it. This resulted in low field harmonics in the body of the magnet as good as those expected after a number of cross-section iterations. Also the magnet had the desired pre-stress on the coils. The first D0 magnet is being used in the RHIC machine. Based on measurements in the first magnet, small adjustments were made in the thickness of the midplane insulation caps and pole shims to compensate the end harmonics and $0.4 \mathrm{~mm}$ radius magnetic rods were inserted in the saturation control holes to reduce small values of saturation induced $b_{2}$ and $b_{1}$ harmonics.

A regular cross-section iteration generally requires a large mechanical change in several wedges and is associated with $(a)$ a change in tooling and $(b)$ a change in the end design. Both of these are time consuming and expensive and are avoided in the above approach. Moreover, this approach requires only small mechanical changes and therefore has a better chance of succeeding. 


\subsection{Integral Transfer Function}

A difference in the integral transfer function (or effective length, $\delta \mathrm{L} / \mathrm{L}$ ) between the magnets built by different vendors has been observed in . HERA magnets [10]. A similar situation was observed in RHIC when different length magnets were built for the insertion regions. Even though the magnets were built by the same vendor (Northrop Grumman Corporation) and on the same design, the integral transfer function was off by a small amount from the expected values.

In most magnets the coil ends are enclosed by nonmagnetic stainless steel laminations instead of magnetic low carbon steel in order to reduce the field on the conductor. It is proposed here that the axial location where the transition between the low carbon steel laminations to stainless steel laminations takes place be used as a parameter to adjust the integral transfer function. This adjustment may be applied after the first few magnets are built and measured. A rough estimate suggests that a $50 \mathrm{~mm}$ adjustment on each end should be adequate in most cases.

An adjustment in the change in transfer function between low field and high field due to iron saturation can be obtained by changing the iron packing factor (i.e. the number of yoke laminations for the same yoke length). This technique has been used in adjusting the integral transfer function in the different length RHIC magnets.

\subsection{Skew Quadrupole in Dipole Magnets}

The presence of a skew quadrupole harmonic in dipole magnets reflects a top-bottom asymmetry. A difference in the yoke length between the top and bottom halves can similarly be used to compensate for this asymmetry [8]. The current dependence in the variation of this harmonic can be adjusted by adjusting the yoke packing factor between the two halves [7].

\subsection{Twist in Field Angle of the Magnets}

A change in field angle (or twist) along the axis of RHIC magnets has been reduced by applying slanted welds on the outer diameter of stainless steel shell. The weld moves the magnet in the direction it is applied. Slanted weld in opposite directions on the two sides of the shell effectively generate a torque which takes out the twist of the magnet.

\subsection{Harmonic Correction after Initial Assembly}

In a perfect design, the field quality is limited only by the errors (tolerances) in parts and assembly. To overcome these limitations, a "tuning shim method" is being used in $130 \mathrm{~mm}$ aperture RHIC insertion quadrupoles [6]. Eight tuning shims of variable iron thickness are inserted to cancel out the measured values of eight harmonics. The test results of the first five magnets show that the harmonics can be corrected within their repeatability and measurement errors.

\subsection{Current Dependence in Field Harmonics}

At high field the harmonics change as a function of current due to iron saturation and also due to Lorentz forces on the coil. A larger variation is expected in RHIC type magnets where the iron contribution to the total field is large. However, the saturation induced harmonics can be minimized by forcing the yoke iron to saturate uniformly with the help of holes, cutouts, etc. in the yoke geometry. A good parameter to examine [9] the iron saturation is $(\mu-1) /(\mu+1)$. The value of this parameter is -1.0 at low field (no saturation or $\mu>1000$ ) and zero at very high field (complete saturation or $\mu=1$ ). The variation in this parameter as a function of azimuthal angle should be small, particularly near the yoke inner radius. In RHIC magnets, despite a large contribution from the yoke, the saturation induced harmonics are small (see references [2] to [5]).

\section{EXPECTED FIELD QUALITY IN SSC-TYPE DIPOLE MAGNETS}

The expected field quality presented in this section for a $50 \mathrm{~mm}$ aperture SSC-type dipole is significantly better than previously assumed in SSC beam tracking studies [11]. The improvement comes from $(a)$ the use of the above methods for controlling field quality $(b)$ a feedback from the measurements in the SSC prototype magnets and $(c)$ the use of revised methods of estimating field errors. The methods that are commonly used for estimating the field harmonics tend to over-estimate the expected field errors. This has been discussed in detail in reference [4] where the possible sources for this overestimate are discussed and the experience with RHIC and SSC magnets is presented. The expected field harmonics are described by (i) the expected mean (ii) the uncertainty in mean and (iii) the expected RMS width or $\operatorname{sigma}(\sigma)$.

The expected integral values of field harmonics in a $50 \mathrm{~mm}$ aperture, 15 meter long, 2-layer SSC-type dipole magnet built along the lines discussed here are given in Table 1 at $10 \mathrm{~mm}$ reference radius for an operating range of 2000 A ( 2 tesla) to 6600 A (6.6 tesla). At injection $(660 \mathrm{~A})$, the allowed harmonics are dominated by the effects of the persistent currents. Since the issue of persistent current is not discussed here, the harmonics are given only at or above $2000 \mathrm{~A}$, where their influence is negligible. The last row gives the expected systematic difference in the integral transfer function or effective length $(\delta \mathrm{L} / \mathrm{L})$ between the magnets built by two or more vendors; the $\sigma$ is for the whole accelerator. For reference, SSC specifications at high field are also given.

The use of the RHIC insertion quadrupole type tuning shims [6] is not assumed here. The tuning shims may 
reduce those harmonics selected for correction by about a factor of five, as long as they are reproducible.

Table 1. The expected normal $\left(b_{n}\right)$ and skew harmonics $\left(a_{n}\right)$ in $50 \mathrm{~mm}$ aperture SSC type dipole magnets at a reference radius of $10 \mathrm{~mm}$ in an operating range of 2000 A to $6600 \mathrm{~A} .\left\langle b_{n}\right\rangle$ and $\left\langle a_{n}\right\rangle$ are the expected mean, $\mathrm{d}\left(b_{n}\right)$ and $\mathrm{d}\left(a_{n}\right)$ are the uncertainty in the mean and $\sigma\left(b_{n}\right)$ and $\sigma\left(a_{n}\right)$ are the expected sigma. The last two columns show the SSC tolerances/specifications for the mean ( $\mathrm{ssc}<>$ ) and sigma (ssc $\sigma$ ) at high field. Since $\left\langle b_{n}\right\rangle$ and $\left\langle a_{n}\right\rangle$ are zero $\mathrm{d}\left(b_{n}\right)$ and $\mathrm{d}\left(a_{n}\right)$ should be compared to the ssc $<>$. The last row gives the expected systematic difference in the effective length ( $\delta \mathrm{L} / \mathrm{L}$ ) between the magnets built by two or more vendors and $\sigma$ for the whole accelerator.

\begin{tabular}{|c|c|c|c|c|c|}
\hline & \multicolumn{3}{|c|}{ SSC Expected Harmonics } & \multicolumn{2}{|c|}{ SSC Tolerances } \\
\hline $\mathbf{n}$ & $\left\langle\boldsymbol{b}_{n}\right\rangle$ & $\mathbf{d}\left(\boldsymbol{b}_{n}\right)$ & $\sigma\left(b_{s}\right)$ & $\operatorname{ssc}<>$ & $\operatorname{ssc} \sigma$ \\
\hline 1 & 0.0 & 0.04 & 0.2 & 0.04 & 0.5 \\
\hline 2 & 0.0 & 0.2 & 0.4 & 0.8 & 1.15 \\
\hline 3 & 0.0 & 0.002 & 0.03 & 0.026 & 0.16 \\
\hline 4 & 0.0 & 0.02 & 0.05 & 0.08 & 0.22 \\
\hline 5 & 0.0 & 0.001 & 0.002 & 0.016 & 0.02 \\
\hline 6 & 0.0 & 0.002 & 0.005 & 0.013 & 0.02 \\
\hline 7 & 0.0 & 0.0004 & 0.001 & 0.01 & 0.01 \\
\hline 8 & 0.0 & 0.0005 & 0.001 & 0.02 & 0.0075 \\
\hline $\mathbf{n}$ & $<a_{n}>$ & $\mathrm{d}\left(a_{n}\right)$ & $\sigma\left(a_{n}\right)$ & ssc $<>$ & $\operatorname{ssc} \sigma$ \\
\hline 1 & 0.0 & 0.04 & 0.5 & 0.04 & 1.25 \\
\hline 2 & 0.0 & 0.02 & 0.15 & 0.032 & 0.35 \\
\hline 3 & 0.0 & 0.01 & 0.07 & 0.026 & 0.32 \\
\hline 4 & 0.0 & 0.002 & 0.02 & 0.02 & 0.05 \\
\hline 5 & 0.0 & 0.002 & 0.008 & 0.016 & 0.05 \\
\hline 6 & 0.0 & 0.001 & 0.005 & 0.013 & 0.01 \\
\hline 7 & 0.0 & 0.0004 & 0.001 & 0.01 & 0.01 \\
\hline 8 & 0.0 & 0.0004 & 0.001 & 0.008 & 0.0075 \\
\hline$\delta \mathrm{L} / \mathrm{L}$ & $\ldots$ & 0.0003 & 0.0004 & -- & 0.0006 \\
\hline
\end{tabular}

\section{CONCLUSIONS}

The expected field quality shown in Table 1 is generally a factor of 2 to 5 better than that previously assumed in SSC beam tracking studies [11]. Such improvements should have some influence on the expected performance of the machine. Most concepts presented/reviewed in this paper have already been successfully tested in RHIC or SSC magnets. The spirit behind the techniques presented here is that simple mechanical adjustments can make significant improvements in the performance of a series of magnets, if they are planned ahead and made part of the initial design.

\section{ACKNOWLEDGMENTS}

Discussions with A. Jain, S. Kahn, G. Morgan, P. Thompson, P. Wanderer and E. Willen on the field quality issues have been very useful and contributed to developing these concepts. The engineering efforts in incorporating these ideas into real magnets from M. Anerella, J. Cozzolino, E. Kelly and J. Schmalzle are also acknowledged. The feedback on field quality requirements in RHIC magnets was provided by S. Peggs and J. Wei. G. Morgan, P. Wanderer and E. Willen made a careful review of this manuscript.

\section{REFERENCES}

[1] R. Gupta, et al., "SSC $50 \mathrm{~mm}$ Dipole Cross Section", Proceedings of the Third International Industrial Symposium on Super Collider (IISSC), Atlanta, USA, pp. 587-600 (1991).

[2] R. Gupta, et al., "Field Quality Improvements in Superconducting Magnets for RHIC", Proceedings of the 1994 European Particle Accelerator Conference, London, UK, pp. 2928-2930 (1994).

[3] R. Gupta, et al., "Field Quality Control Through the Production Phase of RHIC Arc Dipoles", Proceedings of the 1995 Particle Accelerator Conference, London, UK, pp. 2928-2930 (1994).

[4] R. Gupta, "Estimating and Adjusting Field Quality in Superconducting Magnets", Presented at the 1995 LHC Collective Effects Workshop, Montreux, Switzerland; to be published in Particle Accelerators (1996).

[5] R. Gupta, et al., "RHIC Insertion Magnets", Proceedings of the 1991 Particle Accelerator Conference, San Francisco, USA, pp. 2239-2241 (1991).

[6] R. Gupta, et al., "Tuning Shims for High Field Quality in Superconducting Magnets", presented at the Fourteenth International Conference on Magnet Technology (MT-14) at Tampere, Finland (1995).

[7] A. Jain, R. Gupta, P. Thompson and P. Wanderer, "Skew Quadrupole in RHIC Dipole Magnets at High Fields", presented at the Fourteenth International Conference on Magnet Technology (MT-14) at Tampere, Finland (1995).

[8] R. Gupta, "Correcting Field Harmonics after Design in Superconducting Magnets", Proceedings of the Fourth International Industrial Symposium on Super Collider, New Orleans, USA, pp. 773-780 (1991).

[9] R. Gupta, "Field Quality Improvements in Superconducting Magnets", Ph.D. thesis, to be published as a BNL report (1996).

[10]P. Schmuser, "Field Quality Issues in Superconducting Magnets", Proceedings of the 1991 Particle Accelerator Conference, San Francisco, USA, pp. 3741 (1991).

[11]J.R. Sanford and D.M. Matthews, editors, "SiteSpecific Conceptual Design of the Superconducting Super Collider", SSCL-SR-1056 (1990). 
. 


\title{
DESIGN OF A HIGH GRADIENT QUADRUPOLE FOR THE LHC INTERACTION REGIONS
}

\author{
R.C. Gupta, Brookhaven National Laboratory, Upton, New York, USA; \\ R. Bossert, S.A. Gourlay, T. Heger, Y. Huang, J. Kerby, M.J. Lamm, P.J. Limon, P.O. Mazur, \\ F. Nobrega, J.P. Ozelis, G. Sabbi, J. Strait, A.V. Zlobin \\ Fermilab, Batavia, Illinois, USA; \\ S. Caspi, D. Dell'orco, A.D. McInturff, R. M. Scanlan, J.M. Van Oort \\ Lawrence Berkeley National Laboratory, Berkeley, California, USA
}

\section{Abstract}

A collaboration of Fermilab, Lawrence Berkeley National Laboratory and Brookhaven National Laboratory is currently engaged in the design of a high gradient quadrupole suitable for use in the LHC interaction regions. The cold iron design incorporates a two-shell, $\cos 2 \theta$ coil geometry with a $70 \mathrm{~mm}$ aperture. This paper summarizes the progress on a magnetic and mechanical design that meets the requirements of maximum gradient $\geq 250 \mathrm{~T} / \mathrm{m}$, operation at $1.8 \mathrm{~K}$, high field quality and provision for adequate cooling in a high radiation environment.

\section{INTRODUCTION}

Fermilab, Lawrence Berkeley National Laboratory (LBNL) and Brookhaven National Laboratory (BNL), have formed a consortium to provide components for the Large Hadron Collider[1] to be built at CERN in Geneva, Switzerland. A proposed U.S. contribution is the high gradient quadrupoles (HGQ) for the interaction regions. These magnets present some formidable challenges. In addition to the large aperture and high gradient, they must operate at superfluid helium temperature with a beam induced heat load of up to $40 \mathrm{~W}$ per magnet. The design effort has been underway for less than a year and is far from complete. This paper describes the status of the project as of this date.

\section{DESIGN}

\subsection{Magnetic Design}

The HGQ has a two-layer, graded, $\cos 2 \theta$ coil with a $70 \mathrm{~mm}$ diameter bore, magnetic length of $5.5 \mathrm{~m}$ and a gradient in excess of $250 \mathrm{~T} / \mathrm{m}$. The two-layer design was chosen in preference to a 4-layer geometry[2]. Relative to a 4layer design, quench protection is simplified because the inductance is low and the geometry allows for uniformly heating both coils during a quench. This design also rests solidly on our past experience and makes use of existing tooling. The cable uses existing SSC strand, which has a nominal $J_{c}$ of $2,750 \mathrm{~A} / \mathrm{mm}^{2}$. The SSC conductor will be used for model magnets and will

eventually be replaced with improved conductor that is now under development. The goal of the program is to produce strand with a current density of $3,400 \mathrm{~A} / \mathrm{mm}^{2}$. Figure 1 shows the expected short sample (ss) performance using the existing conductor, and Table 1 summarizes the expected magnet performance with existing and improved strand. Table 2 lists the values for the design allowed harmonic coefficients.

Table 1. Short sample (ss) performance parameters.

\begin{tabular}{|l|l|l|l|}
\hline $\mathrm{J}_{\mathrm{C}} @ 5 \mathrm{~T}, 4.2 \mathrm{~K}$ & $\mathrm{G}_{\mathrm{SS}}(\mathrm{T} / \mathrm{m})$ & $\mathrm{I}_{\mathrm{SS}}(\mathrm{A})$ & $\mathrm{B}_{\mathrm{SS}}(\mathrm{T})$ \\
\hline $2,750 \mathrm{~A} / \mathrm{mm}^{2}$ & 251.6 & 13,874 & 10.01 \\
\hline $3,300 \mathrm{~A} / \mathrm{mm}^{2}$ & 260.7 & 14,517 & 10.39 \\
\hline
\end{tabular}

Table 2. Allowed harmonic coefficients (units $10^{-4}$ ).

\begin{tabular}{|c|c|c|}
\hline Component & Collision & Injection \\
\hline b6 & 0.006 & 0.01 \\
\hline b10 & 0.002 & 0.0015 \\
\hline b14 & 0.0015 & 0.001 \\
\hline
\end{tabular}

The two-layer cross section design requires large aspect ratio cables. Samples of both the inner and outer cables have been produced by LBNL and winding studies with the new cables have been successful. Parameters of the improved conductor and cable are given in Tables 3 and 4.

Table 3. Strand parameters for the improved conductor.

$\begin{array}{lc}\text { Alloy } & \text { NbTi } \\ \text { Inner Strand Diameter } & 0.808 \mathrm{~mm} \\ \text { Outer Strand Diameter } & 0.648 \mathrm{~mm} \\ \text { Filament Diameter } & 9 \mu \mathrm{m} \\ \text { Cu/SC Inner } & 1.3: 1 / 1.1: 1 \\ \text { Cu/SC Outer } & 1.8: 1 \\ \text { Twist Pitch } & 10 \mathrm{~mm} \\ \text { Critical Field @ T }=4.2 \mathrm{~K} & 10.4 \mathrm{~T} \\ \text { Critical Field @ T }=1.8 \mathrm{~K} & 13.2 \mathrm{~T} \\ \text { Critical Current Density } & \\ \mathrm{T}=4.2 \mathrm{~K} \& \mathrm{~B}=5 / 8 \mathrm{~T} & 3,400 / 1,500 \mathrm{~A} / \mathrm{mm}^{2} \\ \mathrm{~T}=1.8 \mathrm{~K} \& \mathrm{~B}=8 / 11 \mathrm{~T} & 3,700 / 1,800 \mathrm{~A} / \mathrm{n}^{2} \mathrm{n}^{2}\end{array}$

Alloy

Filament Diameter

Critical Field @ $\mathrm{T}=4.2 \mathrm{~K}$

Critical Field @ $\mathrm{T}=1.8 \mathrm{~K}$

$\mathrm{T}=4.2 \mathrm{~K} \& \mathrm{~B}=5 / 8 \mathrm{~T}$

$=18 \mathrm{~K} \& \mathrm{~B}=8 / 11 \mathrm{~T}$ 
Table 4. Cable Parameters

\begin{tabular}{llc} 
& Cable 1 (Inner) & Cable 2 (Outer) \\
\cline { 3 - 3 } Number of Strands & 38 & 46 \\
Cable Width & $15.4 \mathrm{~mm}$ & $15.4 \mathrm{~mm}$ \\
Keystone Angle & 0.99 degrees & 0.68 degrees \\
Mid Thickness & $1.457 \mathrm{~mm}$ & $1.146 \mathrm{~mm}$
\end{tabular}

The load lines for each coil layer are shown in Fig. 1, indicating the operating margin for a nominal gradient of $235 \mathrm{~T} / \mathrm{m}$ for the SSC conductor performance parameters. The improved conductor will increase the margin to approximately $11 \%$.

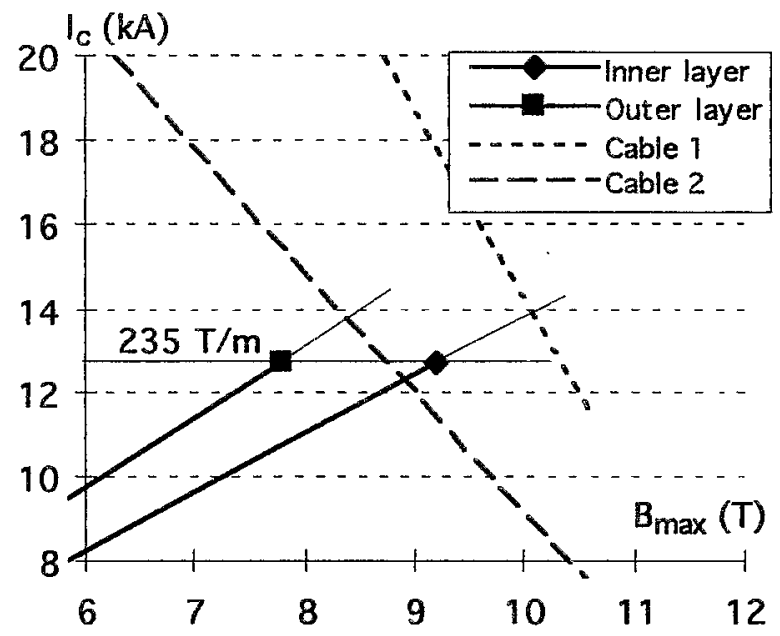

Figure 1. Load lines and critical surfaces for existing strand.

\subsection{Mechanical Analysis}

The HGQ design incorporates the following mechanical features. A strong $20 \mathrm{~mm}$ wide aluminum collar provides significant preload and defines the coil geometry so as to allow warm magnetic measurements to be made. A 2-piece iron yoke surrounds the collared coil and is closed both warm and cold. There is collar-yoke contact under all conditions at the 4 coil mid-planes to reduce coil motion under excitation. Contact is ensured by the larger collar than yoke diameter and the collar deflections due to coil preload. The use of yoke-collar shims is planned to adjust for manufacturing tolerances. A welded stainless steel shell, prestressed to about $200 \mathrm{MPa}$, provides helium containment and compressive load to maintain yoke closure. Following cooldown the compressive force between iron halves reaches at least $2 \times 10^{6} \mathrm{~N} / \mathrm{m}$. This is larger than the radial Lorentz force of $1.14 \times 10^{6} \mathrm{~N} / \mathrm{m}$, so the yoke gap remains closed.

Finite element analysis of this design has been performed[3] using ANSYS to determine limits on the initial collared coil preload and collar-to-yoke spacing. Figure 2 shows azimuthal compressive stress on the coils during assembly, cooldown to $1.8 \mathrm{~K}$, and excitation to maximum gradient for the lower and upper bound cases having a collar-to-yoke interference (warm, undeflected) of $50 \mu \mathrm{m}$. Figure 3 shows lower and upper bounds for the coil prestress as a function of the yoke-collar spacing. For a given spacing, the lower bound is set by the requirement that the coil stress at a gradient of $250 \mathrm{~T} / \mathrm{m}$ be $\geq 14 \mathrm{MPa}$; the upper bound corres ponds to the case when the yoke

Vary Preload +/- 10.7 MPa

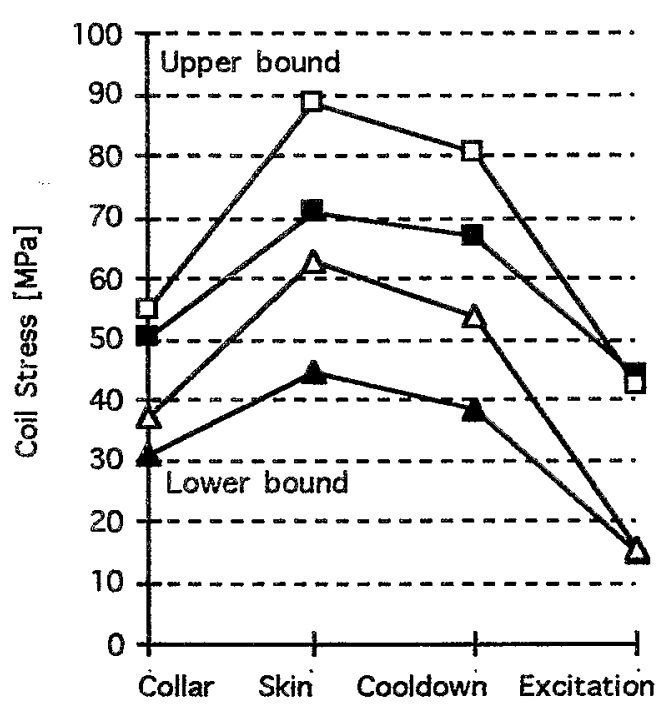

Figure 2. Azimuthal compressive stress on coils during assembly (collaring), cooldown and excitation. Inner (outer) coil stress is shown by open (filled) symbols.

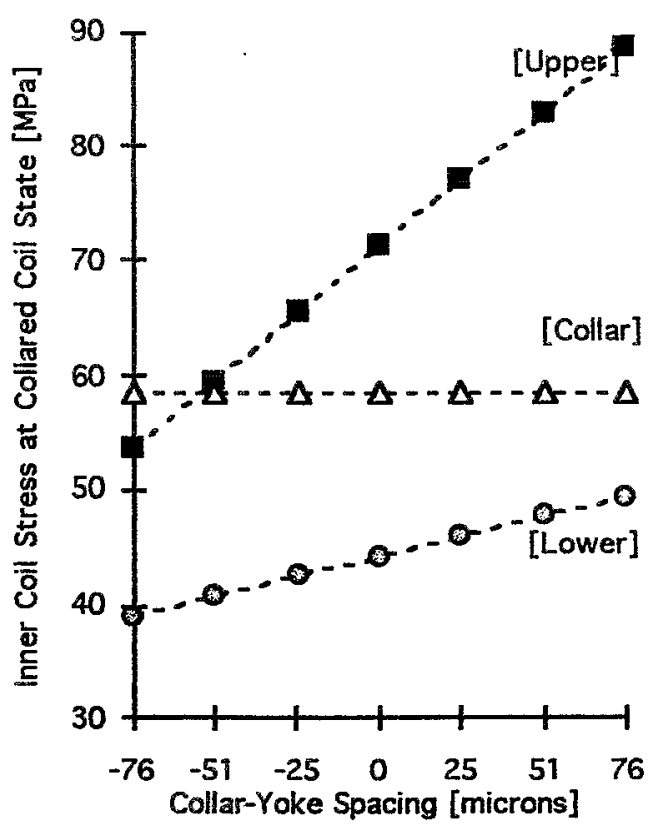

Figure 3. Upper and lower bounds for various collar-yoke spacing and collar stress limit. 
mating surface begins to open at room temperature. The final bound is set by the limit on the peak stress in collar material in the initial collared coil state; it is independent of collar-to-yoke spacing. The range of acceptable preload is between the 3 limits. For the design value of $50 \mu \mathrm{m}$ yoke-collar interference, the range of acceptable collared coil preload is about $\pm 10 \mathrm{Mpa}$. For a given preload, as the collar-yoke spacing increases the collar to yoke force decreases which increases the compressive force between iron halves. The upper bound is a function of that force, so the larger the collar-to-yoke spacing, the larger the upper bound point. Also, all lower bound cases have collar-iron contact cold.

\subsection{Beam Induced Heat Load}

The quadrupole coils in the LHC low- $\beta$ insertions are subject to a constant heat load up to 40 Watts per magnet and a peak energy density as large as $1.2 \mathrm{~mW} / \mathrm{g}$ at the coil mid-plane due to secondary particles from beam-beam collisions at the nominal luminosity[4]. The HGQ cables are wrapped with 25 micron thick Kapton tape with a $50 \%$ overl ap for electrical insulation purposes and one

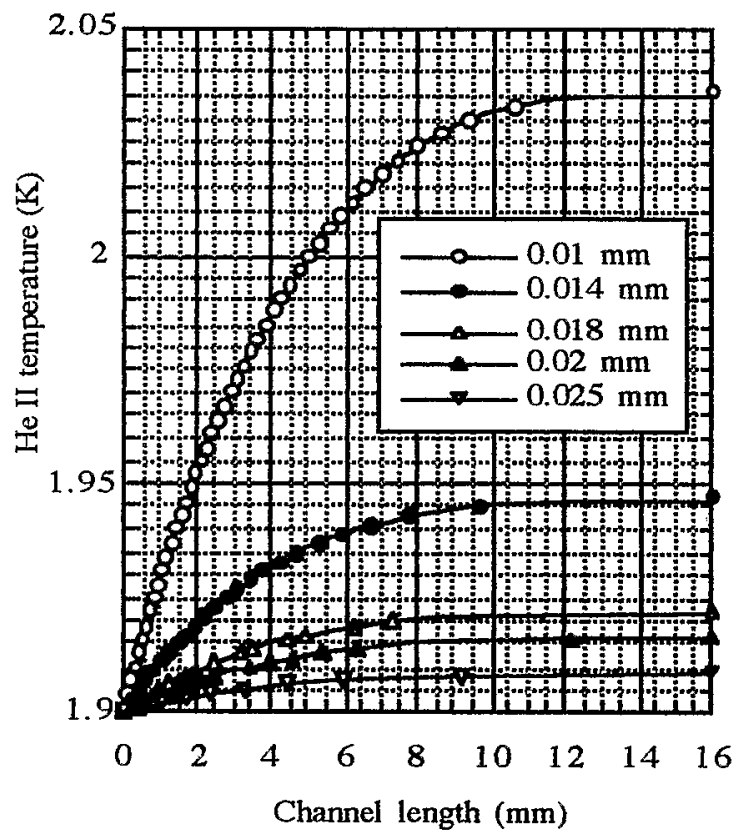

Figure 4. Temperature profile in He II with $2 \mathrm{~mm}$ wide cooling channels and varying channel length and height with a surface heat flux of $4.5 \mathrm{~mW} / \mathrm{cm}^{2}$. layer of spiral wrapped Kapton for mechanical protection and to provide He II cooling channels. The maximum heat load is at the coil mid-plane and the surface heat flux can be calculated based on the surface area in contact with the He II. The cooling channel formed by the second layer of Kapton insulation film will be deformed during the curing process. The temperature profile within the $\mathrm{He}$ II cooling chamber for varying channel gap and length is shown in Fig. 4. A minimum gap of 14 microns is required to keep the He II temperature below $1.95 \mathrm{~K}$.

\section{CONCLUSIONS}

The project is still within its first year and much progress has been made. Several design issues remain to be considered. Tests of the first model are planned for the Spring of 1997.

\section{ACKNOWLEDGMENTS}

BNL is supported by the U.S. Department of Energy under contract No. DE-AC02-76CH00016. Fermilab is operated by Universities Research Association Inc., under contract with the U.S. Department of Energy. The work at LBNL is supported by the Director, Office of Energy Research, Office of High Energy and Nuclear Physics, High Energy Physics Division, U.S. Department of Energy, under contract No. DE-AD03-76SF00098.

\section{REFERENCES}

[1] Large Hadron Collider Conceptual Design, CERN/AC/95-05 (LHC) 1995.

[2] R. Ostojic, T.M. Taylor, and G.A. Kirby, "Design and Construction of a One-Metre Model of the $70 \mathrm{~mm}$ Aperture Quadrupole for the LHC Low- $\beta$ Insertions," Proc. 13th Int. Conf. on Mag. Tech., Victoria, Canada, 1993.

[3] T. Heger, J. Kerby, B. Wands, "HGQ Finite Element Model Verification," Fermilab Technical Support Section Intemal Note TSS-96-003, February, 1996.

[4] N. Mokhov and J. Strait, "Optimization of the LHC Interaction Region With Respect to Beam-Inducod Energy Deposition," presented at the 5th European Particle Accelerator Conference, Sitges, Spain, 10-14 June, 1996. 



\title{
SUPERCONDUCTING COIL COMPRESSION BY SCISSOR LAMINATIONS
}

\author{
Albert Ijspeert, Jukka Salminen, CERN, Geneva, Switzerland
}

\begin{abstract}
A new system of coil compression [1] has been designed which uses iron laminations to transfer the pressure from an outer shrink ring to the coil. The laminations are simple circular discs around the coil with the peculiarity that the rim is slightly eccentric as compared to the coil. Successive laminations are mounted with different angular orientations to oppose their eccentricities. The shrink ring pushes these discs inwards against the coil creating compression by a scissor movement. Tests on mechanical models of single as well as multiple aperture magnets have shown it to work as expected. The system has already successfully been applied to several corrector magnets for LHC. The advantages are low cost (suppression of the usual collars), increased coil compression in particular from cooling down, and field enhancement from having the iron close to the coil.
\end{abstract}

\section{INTRODUCTION}

Coils of superconducting magnets for accelerators need to be compressed to avoid wire movements which would cause quenches. The prestress is in general obtained by collaring the coils under a press or by shrinking rings around the coils. The iron yoke can help increase the pre-compression if pushed inwards by an outer shell but at the expense of subdividing the yoke into different segments. Scissor laminations offer a simple and economic alternative to regular collar systems.

\section{PRINCIPLE}

The principle of the scissor laminations is shown in figure 1 . The yoke is made of a single type of ringshaped laminations stacked around the magnet coil. They have the peculiarity that the circular periphery is designed to be slightly off-centre as compared to the magnet centre $(-0.5 \mathrm{~mm})$. These laminations are mounted with different angular orientations. The outer shrinking shell presses on the wider side of each lamination only and the latter transmits the compressive force to the coil. The wide side of each lamination simply acts as a local spacer between the shrink ring and the coil and the rest of the circular lamination is free. In general the laminations are oriented per pair of opposing eccentricity each pair compressing the coil thanks to the "scissor" effect. Cooling the magnet to cryogenic temperatures enhances the pre-compression of the coil if the shrink ring is of a strongly shrinking

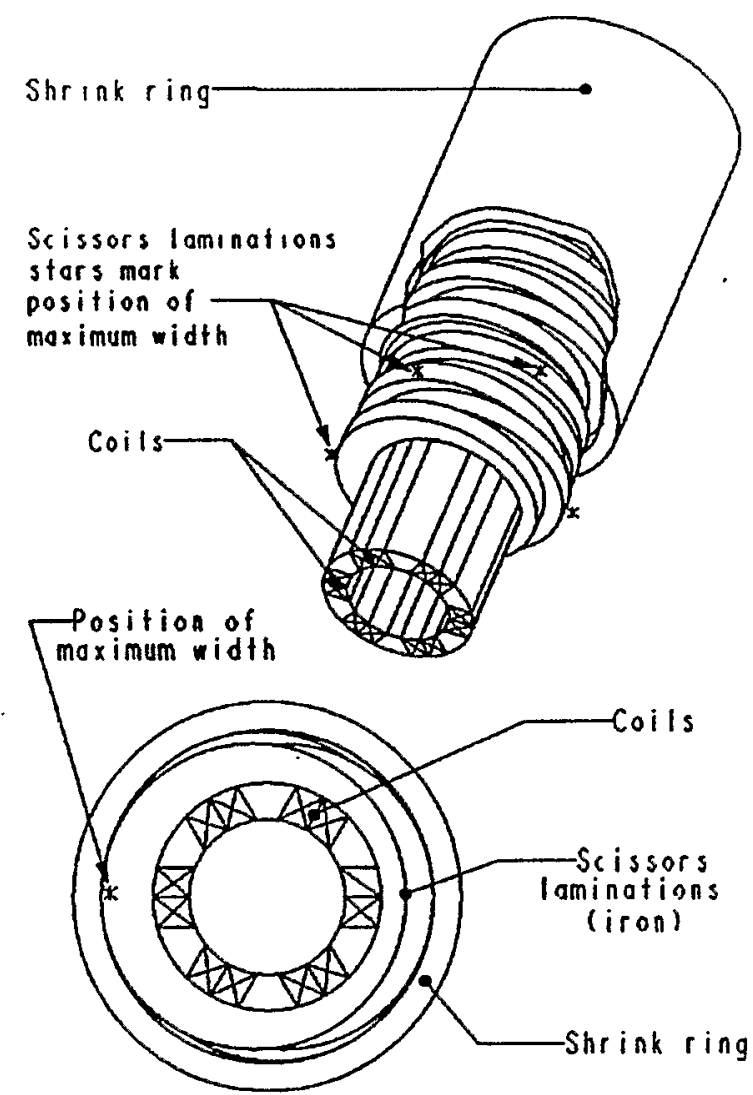

Figure: 1 Principle of Scissor laminations. Shown is sextupole corrector.(eccentricity strongly exaggerated)

material such as aluminium or stainless steel. If desired, one can introduce keys in the laminations to stop the scissor action beyond a certain cooling temperature. Another way to stop it is to choose such an eccentricity that the peripheries of the laminations line up at a well defined cooling temperature. This can be used to build up a circular compression on the laminations that stiffens the yoke; the Lorentz forces that would tend to move the laminations outwards must first overcome that precompression before any elastic movement can occur. The scissor system is readily applicable to magnets with more than one aperture, as shown in sections 4,5 .

\section{SINGLE APERTURE APPLICATIONS}

The method has first been applied to some small superconducting corrector magnets for LHC. Originally their design consisted of a coil shrunk inside an aluminium shrink ring and this assembly was centred inside an iron yoke by means of keys [2]. This has now been simplified by packing the coil inside scissor laminations and shrinking the ring around this assembly. 
The advantages are that the iron has been brought close to the coil boosting the field, that the shrink ring acts now at a larger diameter and is therefore easier to shrink and also yields more prestress when cooling down, and finally that it suppresses the expensive machining of keyways. Three different corrector magnet prototypes have been built along these lines, one sextupole and one decapole by INDAR, Spain and another sextupole by CERN [3]. Table 1 gives their data. All three magnets use the same enamelled wire of $0.61 \times 1.13 \mathrm{~mm}$ metal cross section. The first magnets are made with vacuum

Table:1 Parameters of the three corrector prototypes

\begin{tabular}{|l|r|r|r|r|}
\hline & & INDAR & INDAR & CERN \\
\hline & & sextupole & decapole & sextupole \\
\hline Magnet length & $\mathrm{mm}$ & 150 & 75 & 150 \\
\hline Nominal current & $\mathrm{A}$ & 383 & 346 & 625 \\
\hline Critical current (4.2K) & $\mathrm{A}$ & 765 & 945 & 993 \\
\hline Peak field at crit. current & $\mathrm{T}$ & 5.2 & 4.1 & 3.26 \\
\hline Coil Inner Diameter & $\mathrm{mm}$ & 56 & 56 & 56 \\
\hline Coil Outer Diameter & $\mathrm{mm}$ & 76 & 71 & 61 \\
\hline Number of radial layers & & 8 & 6 & 2 \\
\hline Number of turns & & 112 & 48 & 26 \\
\hline Dismeter over coil insulation & $\mathrm{mm}$ & 85.5 & 78.9 & 66 \\
\hline Scissors lamination I.D. & $\mathrm{mm}$ & 86.2 & 79 & 66.2 \\
\hline Scissors lamination O.D. & $\mathrm{mm}$ & 115 & 95.2 & 89.4 \\
\hline Largest lamination width & $\mathrm{mm}$ & 15.1 & 8.4 & 12 \\
\hline Smallest lamination width & $\mathrm{mm}$ & 13.8 & 7.7 & 11.2 \\
\hline Lamination thickness & $\mathrm{mm}$ & 0.8 & 0.8 & 1 \\
\hline Angular orientations & & 6 & 10 & 6 \\
\hline Aluminium shrink ring I.D. & $\mathrm{mm}$ & 115.4 & 95.3 & 89.88 \\
\hline Shrink ring O.D. & $\mathrm{mm}$ & 140 & 106 & 100 \\
\hline Interference on diameter & $\mathrm{mm}$ & 0.35 & 0.44 & 0.12 \\
\hline Average coil prestress 300 K & $\mathrm{MPa}$ & -35 & -46 & -31 \\
\hline Expected coil prestress 4.2 K & $\mathrm{MPa}$ & -53 & -47 & -36 \\
\hline
\end{tabular}

impregnated coils with several layers of winding. The last magnet is made in a more economical way with a two layer coil wetted with epoxy during the winding process. The coils of these magnets are protected with a $2.5 \mathrm{~mm}$ thick glassfiber epoxy layer and the laminations are directly placed around this insulation layer. The design did not incorporate a mechanical stop. The sextupole laminations were oriented in six angular positions. The circular coil assembly will therefore undergo a slightly hexagonal deformation calculated to be less than $0.03 \mathrm{~mm}$ on the radius which does not compromise the field quality. If necessary one can shape the opening in the laminations to compensate for this. This also implies that the coil is supported by local forces repeated every 4.8 or $6 \mathrm{~mm}$ over the length. The decapole laminations were oriented in ten angular positions and the pitch between every tenth lamination is $8 \mathrm{~mm}$. The magnets have been trained at $4.2 \mathrm{~K}$ at CEDEX in Spain (Fig. 2). The first two magnets show one quench at or below the nominal current. The third magnet starts training well above the nominal current. Compared to other designs with similar impregnated coils the training is similar or even quicker which shows that the laminations give a successful pre-stress.

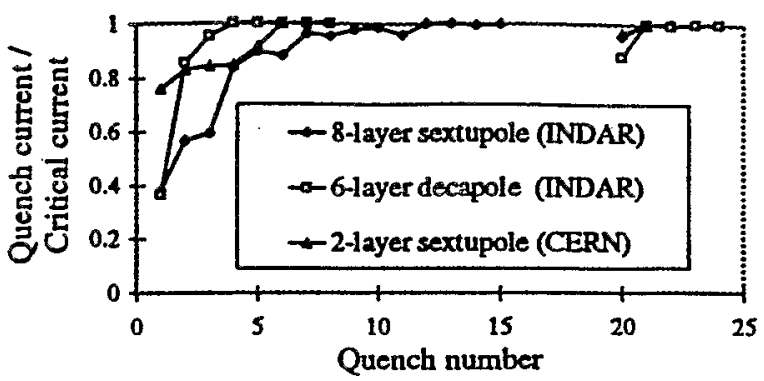

Fig: 2 Training and re-training of the three corrector prototypes

\section{MULTI APERTURE MODEL}

The method can readily be applied to magnets with a multitude of apertures. A dummy assembly of a twin aperture model has been built and tested to measure the application to multi aperture magnets (Fig.3). In contrast to the single aperture case, one cannot turn the scissor lamination to any desired orientation. However, if the multiple apertures are symmetric in the $X$ and the $Y$ planes a single type of lamination can be used in four different positions: a starting position, an opposing position obtained by turning the plate over 180 degrees, and a third and fourth position by tuming the first two plates upside-down. The angle of eccentricity can be chosen to 45 degrees as we did in this model for equal prestress in all four directions or it can be chosen differently to obtain a dominant pre-stress in one particular direction. The plate thickness was $5 \mathrm{~mm}$ and aluminium dummy coils were used as well as an aluminium shrink ring. The stresses as found from diameter measurements show (Fig.4) that the coil compression increases strongly during the cooldown and the alignment of the plates occurs at about $130 \mathrm{~K}$. Beyond this temperature the shrinkage of the shrink ring is not acting on the coil anymore and the coil is slightly

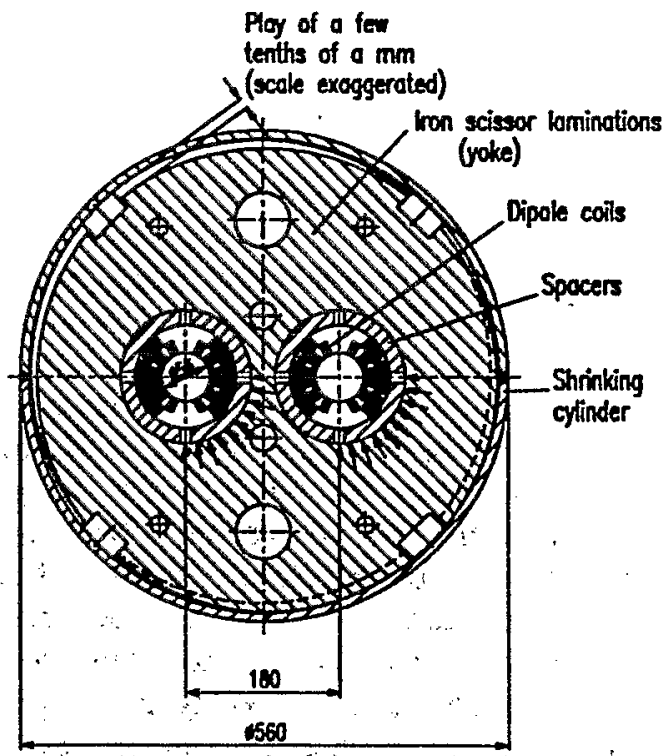

Fig:3 Mechanical model of twin aperture magnet 
de-compressing. Several heat cycles showed that the results are repeatable. The pressure on the coil before and after a heat cycle are practically identical ( $+/-$ $10 \%$ ) which means that frictional effects are very small. The model has several times successfully been disassembled and re-assembled, once using a real coil.

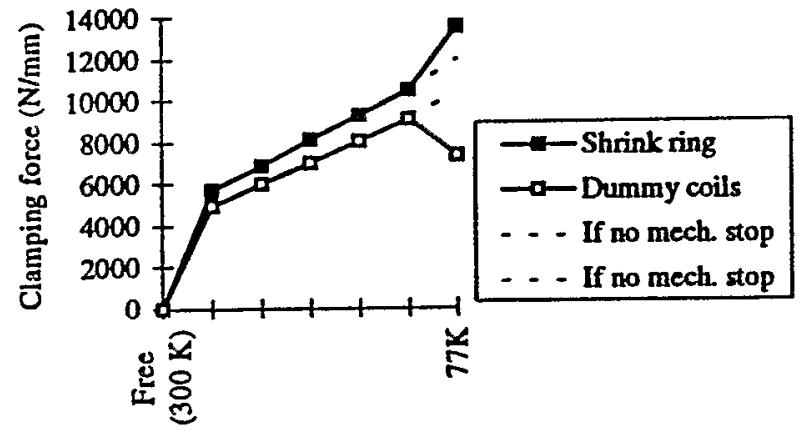

Fig:4 Clamping force in the twin aperture model (corresponding to coil stress of $40 \mathrm{MPa}$ at $300 \mathrm{~K}$ and 60 $\mathrm{MPa}$ at $77 \mathrm{~K}$ for chosen interference)

\section{TWIN APERTURE DESIGN}

As a possible application to a multi aperture yoke a tentative design has been made for the LHC main dipole. Two questions turned up: Can one slip the laminations over the not yet collared coil which has dimensions a few millimetres larger than the compressed dimensions and how close can one bring the iron to the coil without losing the field homogeneity due to saturation of the iron at the very high field of 8.4 Tesla? To satisfy these demands we use the fact that each lamination only needs to touch the coil over the 90 degree angle where the pressure is exerted. The shape of the rest of the hole in the lamination can be at a larger radius to obtain the play necessary for the assembly. Magnetic calculations showed that the multipoles caused by local saturation in the iron can be reduced to less than $10^{-4}$ of the main field over the whole range of excitation by shaping the hole of the iron lamination elliptical and by adding holes to the lamination in the correct positions (Fig.5). The calculations showed that the field homogeneity is not influenced by the iron quality at least less than in the present design. A cost estimation showed that the suppression of the usual collars may lead to an interesting cost saving on such a magnet. The assembly can be made by securing packages of laminations in the open position using keys, slip the packages over the coils, take the keys out, pre-compress the coils moderately under a press and maintain the compressed position introducing corresponding keys. The skin can then be welded around the magnet and the keyways are such that they accept the additional compression during cooldown loosening the keys.

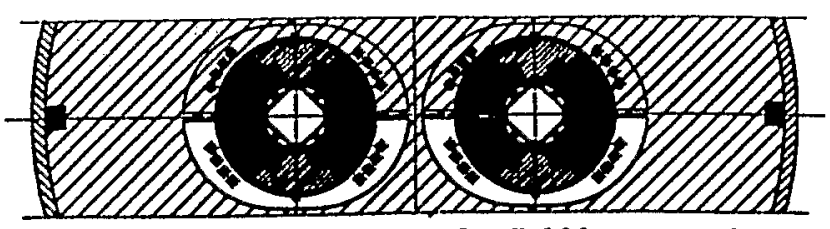

Fig:5 Elliptic lamination holes for field homogeneity

\section{CONCLUSIONS}

It has been shown that the scissor laminations form a practical and elegant way to transmit the compressive force from the shrink ring to the coil body. The yoke is built with a single type of lamination and does not need a subdivision in segments. The system allows to chose freely the desired direction of the prestress on the coil by orienting the laminations accordingly. It can replace the usual collars leading to non-negligible savings. It permits to bring the iron close to the coil enhancing the magnetic field and allowing to reduce the outer diameter of the yoke. Finally it allows to "collar" the coil at a relatively low compression because an additional compression can be obtained from the differential contractions during the cooldown thus avoiding damage to the insulation by over-stress at room temperature and reducing the size of the necessary press. The system has been tested on mechanical models and has already successfully been applied to several types of corrector magnets.

\section{ACKNOWLEDGEMENTS}

We would like to thank G. Laurent, H. Meyer and G. Trinquart for the design work, J. Mazet, J. Perez and D. Regin for the development work and the fabrication of the CERN prototype. Thanks go to J. Etxeandia and J. Garcia from INDAR Spain for the fabrication of the two other prototypes guided by E. Baynham, B. Coombs and D. Evans from DRAL England. Finally thanks go to L. Garcia Tabares, M. Bajko, J. Calero and M. Saenz de Buruaga of CEDEX Spain for the training tests at $4.2 \mathrm{~K}$.

\section{REFERENCES}

[1] A. Ijspeert, 'Compressing coils of single and multiple aperture superconducting magnets with "Scissors" laminations: principles and results of tests on mechanical models', LHC Note 292, CERN, October 1994.

[2] D. Baynham, R, Coombs, A. Ijspeert, R. Perin, 'Design of superconducting corrector magnets for LHC', IEEE Trans. on Magn., Vol.30, No 4, pp1823-1826, July 1994.

[3] J. Salminen, A. Ijspeert, 'Superconducting sextupole corrector magnet for the LHC main dipoles', this conference. 


\title{
Construction and Tests of a Model of the LHC Superconducting Corrector Magnet MDSB V
}

\author{
A. Ijspeert, R. Perin, CERN, European Organization for Nuclear Research, Geneva, Switzerland. \\ E. Baynham, P. Clee, R. Coombs, D. Evans, RAL, Rutherford Appleton Laboratory, Chilton, England. \\ M. Begg, D. Landgrebe, Tesla Engineering, Storrington, England
}

\begin{abstract}
A full-scale model of the $1.25 \mathrm{~m}$ long MDSBV (Magnet Decapole Sextupole Bending Vertical) correction magnet for the Large Hadron Collider (LHC) [1] has been constructed and is currently being tested. The model contains the desired dipole and sextupole but not the decapole which was decided upon later. The magnet was built in a very compact way by placing the dipole coil around the sextupole coil. The two coils were vacuum impregnated and prestressed by shrink-fitted aluminium rings. The design took into account the high positional accuracy requirements for the coils and incorporated manufacturing techniques which are compatible with mass production methods, as approximately 800 of these magnets will be requirec for the LHC. The model is being tested in liquid helium at the temperature of $4.2 \mathrm{~K}$ and will be tested later at $2.0 \mathrm{~K}$. The paper describes the construction, the experience gained during assembly, the test conditions and gives the first test results.
\end{abstract}

\section{INTRODUCTION}

The LHC lattice foresees two correction magnets in the standard half cell of each of the two rings, a first one called MDSBH (Magnet Decapole Sextupole Bending Horizontal) or MDSBV (idem Bending Vertical) is placed close to the quadrupoles and a second one, MDOS (Magnet Decapole Octupole Sextupole) in the centre of the half cell between the dipoles. The MDSBH and the MDSBV contain respectively a horizontal and a vertical 1.5 $T$ dipole coil for orbitcorrection. In addition, both contain a 4500

- $T / \mathrm{m}^{2}$ sextupole coil to compensate the sextupole errors from the main dipole magnets and to correct the lattice chromaticity, and a $3.2 \times 10^{7} \mathrm{~T} / \mathrm{m}^{4}$ decapole coil to correct the decapole errors from the main dipole magnets. The other corrector magnet (MDOS) will be a combination of a sextupole of $1600 \mathrm{~T} / \mathrm{m}^{2}$, an octupole of $1.8 \times 10^{5} \mathrm{~T} / \mathrm{m}^{3}$ and a decapole of $1.4 \times 10^{8} \mathrm{~T} / \mathrm{m}^{4}$; the design of which is discussed elsewhere [2].

The MDSBV model has been designed with a sextupole of $8000 \mathrm{~T} / \mathrm{m}^{2}$ and does not yet contain the decapole. Its cross-section is shown in Fig. 1. In addition to the coils and the shrink-fitted rings one sees the systcm of keys which centres the coil assembly in the solid iron yoke and absorbs the differential contraction between the two components.

\section{CONSTRUCTION OF THE COILS}

The design parameters of the sextupole and the dipole coils are given in Table 1.

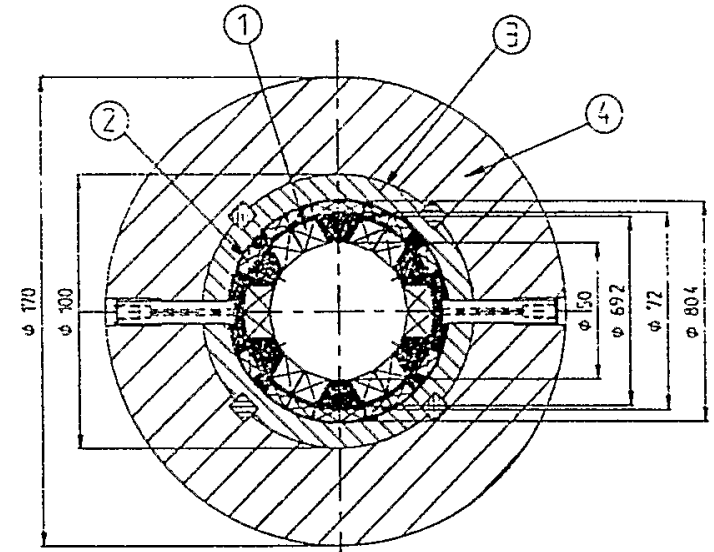

Fig. 1. Cross-section of MDSBV model magnet 1. Sextupole coil, 2. Dipole coil,

The sextupole coil was wound with a superconducting wire of rectangular cross-section, around a copper central island which had previously been insulated with $0.2 \mathrm{~mm}$ of $\mathrm{G}-10$. End spacers were put in after every second layer to dilute the end field and to absorb the space taken by the joggles. It was then impregnated with epoxy resin in a mould. From the tests carried out on trial coils it appeared that the PVA insulation on the wire expanded during the heating of the mould thereby blocking the interturn spaces and preventing the resin from fully penetrating

TABLE !

COIL PARAMETERS OF MDSBV MODEL MAGNET

\begin{tabular}{|l|l|l|}
\hline & Sextupole & Dipole \\
\hline Wire dimension (metal) & $0.58 \mathrm{~mm} \times 1.08 \mathrm{~mm}$ & Diam. 0.29 mm \\
Wire dimension (overalll) & $0.70 \mathrm{~mm} \times 1.20 \mathrm{~mm}$ & Diam. 0.35 mm \\
Copper/NbTi ratio & 1.6 & 4.5 \\
Insulation (enamel) & $0.06 \mathrm{~mm}$ PVA & $0.03 \mathrm{~mm}$ PVA \\
Nominal current & $458 \mathrm{~A}$ & $47 \mathrm{~A}$ \\
\%o of short sample & 61 & 83 \\
Number turns/coil & 104 & 1332 \\
Stored energy & $6.2 \mathrm{~kJ}$ & $5 \mathrm{~kJ}$ \\
Inductance & $59 \mathrm{mH}$ & $5.7 \mathrm{H}$ \\
Length straightsectn. & $1 \mathrm{~m}$ & $1 \mathrm{~m}$ \\
\hline
\end{tabular}


the coil. The impregnation and curing of the actual coils wats therefore carricd out at a lower temperature to avoid this problem.

The coil wire resistance was measured and the ground insulation tested, before and after winding the coil and also after impregnating with resin.

The dipole coil was wound with a superconducting wire of round cross- section (diam. $0.29 \mathrm{~mm}$ ), again around a copper central island. Winding trials showed that it was difficult to wind such a thin wire in a regular pattern. The wire was therefore prefabricated, on a special machine, into a twelve parallel wire ribbon, bonded together with a meniscus of epoxy resin between each wire. To ensure that subsequent turns did not nest in the grooves of the previous turn, the ribbon was flattened between rollers reducing the overall thickness from $0.35 \mathrm{~mm}$ to $0.32 \mathrm{~mm}$. This produced flats on the wires and ensured that the turns would stack in a regular manner. As the insulation on the ribbon was very thin, it was important to carry out the work in a clean area to avoid dirt particles getting between the layers and puncturing the insulation.

The coil basically consists of two blocks, separated by a copper spacer. Three other sub-blocks had to be created to allow the coil to follow the curvature of the cylinder. These were created by wedge-shaped longitudinal spacers. The latter and the end spacers were fabricated from filled epoxy resin.

The series connections of the twelve wires in the ribbon were - made after the coil had been impregnated with epoxy resin. This had the advantage of being able to carry out inter-wire insulation tests at all stages during the fabrication.

The connections were made by fanning out the ribbon at the ends into individual wires and removing the PVA insulation. Connecting wires were then twisted together and soft-soldered. Each joint was then soldered to a printed circuit board to provide mechanical support and additional heatsink by the soldered tracks. Table 2 gives the resistances of different connection samples, meitiured at $4.2 \mathrm{~K}$. Fig. 2 show the execution of the series connections of the dipole coil pancakes.

TABIL: 2

JOLN RESISTANCES OF PALRS OF DITOLE WIRES

Measured resuls ior a joint length of $10 \mathrm{~mm}$. (Only one sample of each combination was mezsured. therefore can unly be used as guideline figures)

\begin{tabular}{|c|c|c|c|}
\hline \multirow[t]{2}{*}{ Methox of bonding } & \multicolumn{3}{|c|}{ Joint layout } \\
\hline & $: \bar{T}$ Tried & $\begin{array}{l}\text { Twisted and } \\
\text { wire-wrapped } \\
\text { with Cu wire }\end{array}$ & $\begin{array}{l}\text { Twisted and } \\
\text { wirc-wrapped } \\
\text { with s.c.wirc }\end{array}$ \\
\hline $\begin{array}{l}\text { Pre-tinned wite, } \\
\text { soldered }\end{array}$ & $3.610^{\circ} \Omega 2$ & $2.610^{.7} \Omega$ & $1.010^{\circ} \Omega$ \\
\hline $\begin{array}{l}\text { Bare wire. } \\
\text { soldered }\end{array}$ & .1 .21052 & $0.510^{7} \Omega$ & $0.610^{7} \Omega 2$ \\
\hline $\begin{array}{l}\text { Bare wire. } \\
\text { not soldered }\end{array}$ & $2.210^{4} \Omega$ & $2.010^{4} \Omega$ & $6.610^{+} \Omega$ \\
\hline
\end{tabular}

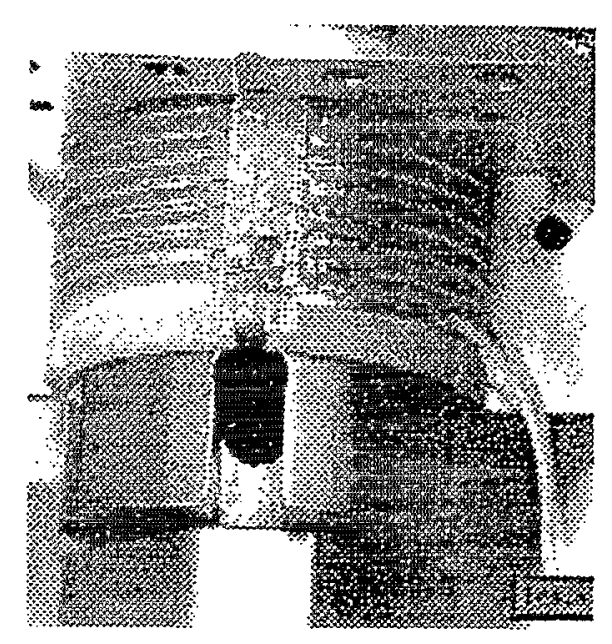

Fig. 2. The soldered joints of the dipole pancakes

\section{ASSEMBLY OF THE COILS}

The sexiupole coils were assembled on a retractable manitr: using dowel pins. The inter spaces between the coils were lilled with glass fibre and, at seven places over the length of the coil. bands of pre-impregnated glass fibre were wound around the assembly and cured at room temperature. The bands werc clumpe:d in accurately located mould tools such that after curing the outer surface of the bands provided a precise diameter onto which the dipole coils were located concentrically with the sextupole coils within the required tolerance. The bands also guarantecd dic angular positions of the sextupole coils such that the dowel pins: could be withdrawn. This was necessary to allow the final extraction of the central mandrel. The series connections were then made between the sextupole coils by soldering the connening pairs of wires into u-shaped copper channels, the latter forming an additional heatsink.

The sextupole coil assembly was finished by filling the spak:s: between the bands with glass fibre tape which was then filled will: resin during the final impregnation of the complute coil triv; ; bly.

The dipole coils were assembled on the cutside dinm, st:- in

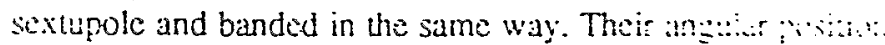
relative to the sextupole coils was maintaned with boling mounted on the two ends. Once these bands were curst the tooling was removed, the series connections were madc, and the interspaces between the bands filled with glass fihre iape. Fuati: the whole assembly was impregnated in a mould. The dianneiter of the mould had been made such that the coil diameter wintid in: precise enough for the titting of the aluminium shrink ring: without any machining of the coil assembly. It appeared to to necessary, however, to polish the outside of the coil assembly tis the roughness of the surface was not acceptable for the shrink fitting process. (The precision obtained during assembly is typically \pm 1.5 milliradian for the angles of the pole mating faces ant: $\pm 0.05 \mathrm{~mm}$ on the radii.) 
The shrink fit was done after measuring the diameter of the impregnated coil assembly and machining the aluminium shrink rings to the desired interference fit. Table 3 gives the measured fit at room temperature as well as at $77 \mathrm{~K}$ and also lists the deduced pre-stress and contraction coefficients at liquid nitrogen temperature.

Finally, the keyways were machined in the aluminium rings to centre the coil assembly in the yoke. The latter was planned to be made of a simple thick-walled iron tube. However, it was not possible to machine the keyways over the one-metre length within the desired precision of $0.05 \mathrm{~mm}$ and therefore the yoke had to be subdivided into short lengths. For a series production a laminated yoke made with precise stampings will therefore be more appropriate.

The power connections to the current leads were made on the periphery of the magnet by clamping the lead against the coil wire (Fig. 3).

The input and output wires of the sextupole coil were brought out of the coil assembly up to the clamps on the periphery and were backed up with a copper strip. The input and output wires of the dipole were first soldered to a thicker wire of the type used for the sextupole coil and then brought from the coil to the clamps as in the case of the sextupole. Shunt resistors, to protect the magnet against an accidental open circuit, were mounted on the outside of the yoke and were directly connected to the coil side of the connection clamps.

\section{THE TESTS}

A first test has been carried out at Rutherford Laboratory at a temperature of $4.2 \mathrm{~K}$. This test, where the magnet was suspended in a vertical cryostat, included the measurement of the magnetic field (by means of Hall probes), the voltages on each individual sextupole coil and the voltages on each dipole pancake. As can be seen on the load lines (Fig.4 and Fig. 5), the sextupole was expected to reach its nominal field even at this temperature but the dipole was not. The first part of the test consisted of running up the sextupole magnet and the dipole magnet separately. In the

TABLE 3

PRE-STRESS FROM SHRNK FT AND COOLDOWN

\begin{tabular}{|l|c|}
\hline Shrink fit & \\
O.diam. impregnated coil (R.T.) & $82.482 \pm 0.009 \mathrm{~mm}$ \\
I.diam. aluminium rings & $82.360 \pm 0.010 \mathrm{~mm}$ \\
Idem after shrink fit & $82.422 \pm 0.010 \mathrm{~mm}$ \\
Pressure between & $9.3 \mathrm{~N} / \mathrm{mm}^{2}$ \\
\hline ring and coil & \\
\hline Conldown to $77 \mathrm{~K}$ & $82.103 \mathrm{~mm}$ \\
I.diam. aluminium rings & $82.066 \mathrm{~mm}$ \\
I.diam. free aluminium ring & $3.6 \times 10^{3} \mathrm{~mm} / \mathrm{mm}$ \\
Thermal contraction aluminium & $4.1 \times 10^{-3} \mathrm{~mm} / \mathrm{mm}$ \\
Thermal contraction coil & \\
Remaining pressure & $6.8 \mathrm{~N} / \mathrm{mm}^{2}$ \\
\hline
\end{tabular}

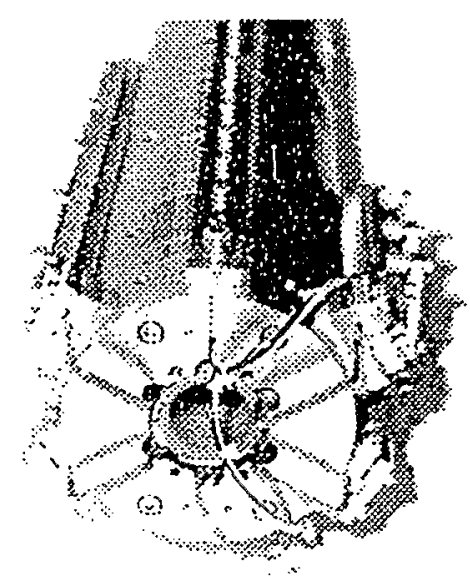

Fig. 3. The power connections of the magnet

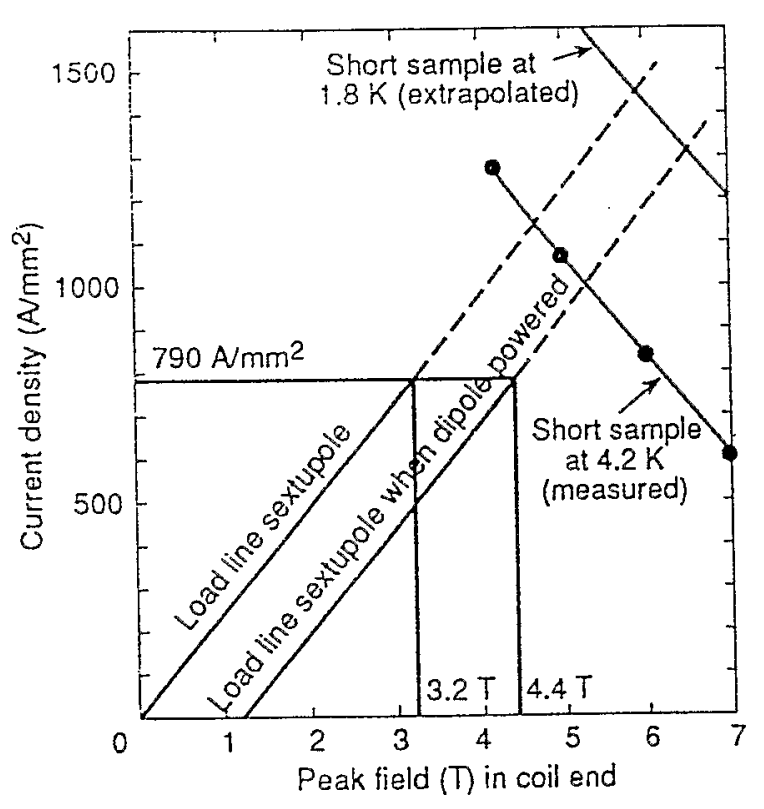

Fig.4. The load lines of the sexlupole.(Conductor parameters given in Table 1)

second part one magnet was powered and the other run up to see the limitations introduced by the coexistence of the two magnetic fields. This second part was repeated after changing the polarity of one of the magnets. In this case the field distribution changes to its mirror image and the peak fields will therefore be found in locations symmetrical to those in the previous condition. The dipole performance is shown in Fig. 6 . In stand-alone operation, it trained from $44 \%$ to $80 \%$ of short sample current (corresponding to a field of $1.44 \mathrm{~T}$ ) in 32 quenches and was still improving. With an additional sextupolar field, the peak field in the dipole coil increases and occurs in another location. Therefore it trained again but more rapidly. After changing the polarity, it seemed to continue the same training curve. The sextupole performance is shown in Fig. 7. In seven quenches it trained from 52\% to $74 \%$ of short sample current (corresponding to a gradient of $8500 \mathrm{~T} /$ $\mathrm{m}^{2}$ ). The training was stopped at this point to avoid overstressing 


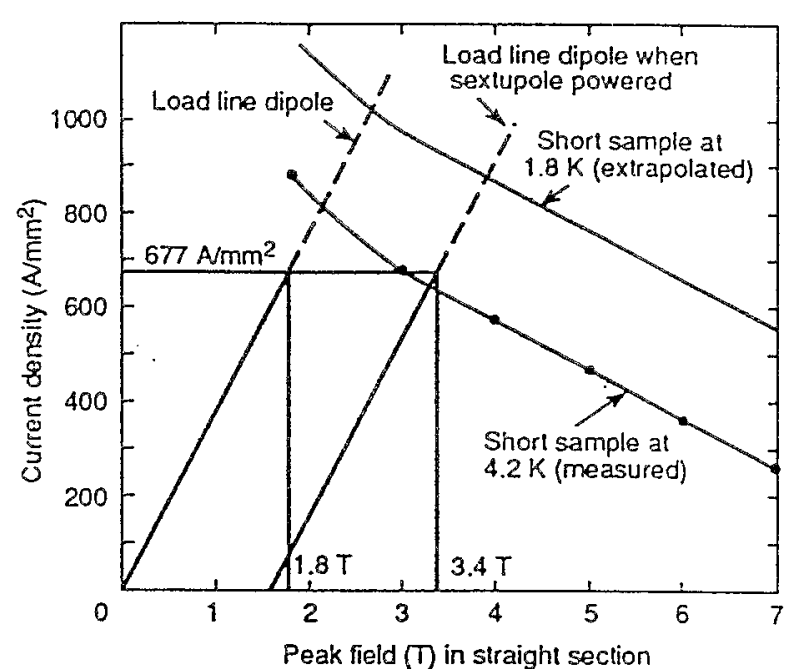

Fig. 5. The load lines of the dipole. (Conductor parameters given in Table I)

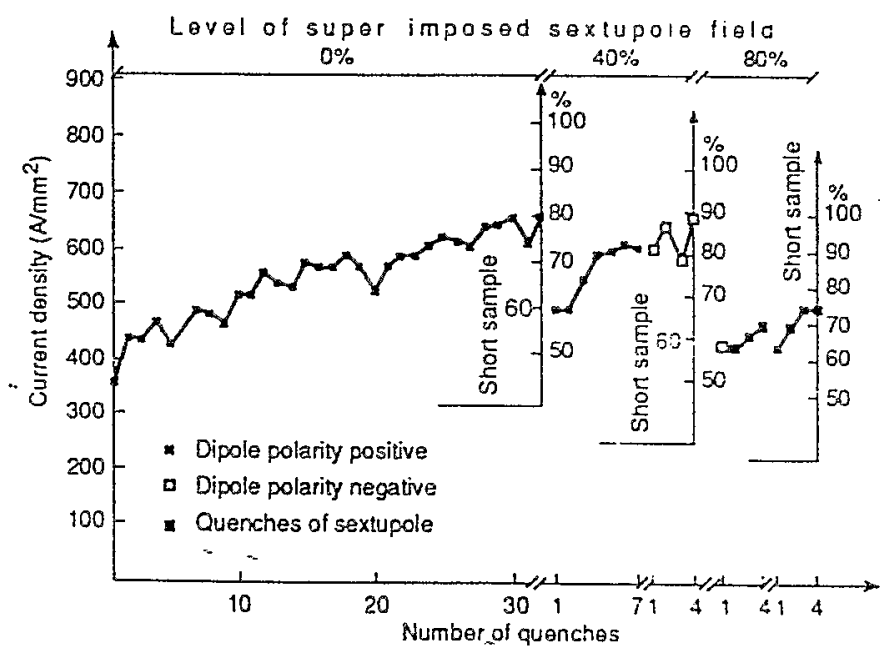

Fig. 6. Training of dipole at $4.2 \dddot{\AA}$

the coil as its design gradient was $8000 \mathrm{~T} / \mathrm{m}^{2}$. An additional dipole field caused a new training to approach a similar point as compared to short sample current. A second test will follow at CERN and will include the measurement of the field harmonics in the straight section and in both ends. Although this test will finally be done at the LHC operating temperature of $1.8 \mathrm{~K}$, the magnet will first be tested at $4.2 \mathrm{~K}$ to see whether it will directly reach the present performance again or whether it will re-train.

\section{CONCLUSIONS}

The MDSBV magnet model, built by "nesting" the sextupole coil inside the dipole coil, has been constructed and successfully tested at $4.2 \mathrm{~K}$. The dipole, designed for a field of $1.5 \mathrm{~T}$ at a temperature of $1.8 \mathrm{~K}$ was brought to $1.44 \mathrm{~T}$ in stand-alone mode and to $1.08 \mathrm{~T}$ when the sextupole was powcred at $80 \%$ of nominal field. The sextupole, designed for $8000 \mathrm{~T} / \mathrm{m}^{2}$ at $1.8 \mathrm{~K}$, was brought

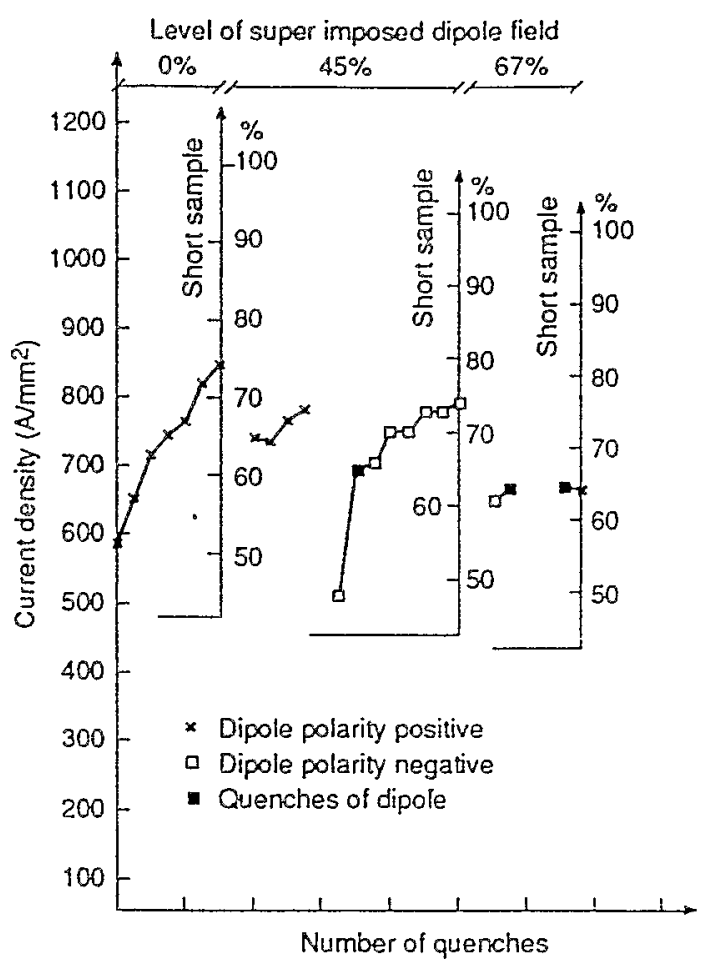

Fig. 7 Training of sextupole at $4.2 \mathrm{~K}$

to $8500 \mathrm{~T} / \mathrm{m}^{2}$ in stand-alone operation and to $6800 \mathrm{~T} / \mathrm{m}^{2}$ when the dipole was powered to $67 \%$ of its nominal field. The dipole showed more training then the sextupole, maybe due to the large number of turns (1332) per pole. The result seems to confirm that the magnets, after training; may run at $70 \%$ of short sample current. However, a second test at $4.2 \mathrm{~K}$ is necessary to check whether the magnets will re-train. A final test at the LHC operating temperature of $1.8 \mathrm{~K}$ is planned in the near future.

\section{ACKNOWLEDGMENTS}

We would like to acknowledge the design effort of M. Purr (Tesla) and R. Mauris and H. Nemoz (CERN), the fabrication by J. Treasure (TESLA), the quench studies by $D$. Hagedom and $F$. Rodriguez-Mateos (CERN), the testing by R. Puffet (R.AL) and B. Vullierme (CERN), the preparation of the paper by the DTP. 4 Centre (CERN) and last but not least J. Whealley, D. Willis and $T$. Kitchin of Tesla Engineering who strongly stimulated and suppored this work.

\section{REFERENCES}

[1] A. Ijspeer, R. Perin. E. Baynham, P. Clec. R. Coombs, J. Wheatley. D. Willis, "Development of a supercondueting sex. tupole-dipole corrector magnet," Proc. llth Int. Conf. on Magn.Techn.(MT-11), Tsukuban Japan. 1989. London. Elsevier. Vol 1.pp 200-205.

[2] E. Baynham, R. Coombs, P. Clec, A. Ijspeert, R. Perin, "Design of a superconducting multipole corrector for LHC," unpublished, submitted to this conference. 


\title{
AUTOMATED DESIGN OF A CORRECTION DIPOLE MAGNET FOR LHC
}

\author{
Mikko Karppinen, Stephan Russenschuck, Albert Ijspeert, \\ CERN, Geneva, Switzerland
}

\begin{abstract}
A correction dipole magnet, with a horizontal dipole nested inside a vertical dipole has been designed and optimized linking together different electromagnetic software and CAD/CAM systems. The necessary interfaces have recently been established in the program ROXIE [1] which has been developed at CERN for the automatic generation and optimization of superconducting coil geometries. The program provides in addition to a mathematical optimization chest, interfaces to commercial electromagnetic and structural software packages, CAD/CAM and databases. The results from electromagnetic calculations with different programs have been compared. Some modeling considerations to reduce the computation time are also given.
\end{abstract}

\section{INTRODUCTION}

The low- $\beta$ dipole corrector, MCBX, is a single-bore, $0.6 \mathrm{~m}$ long magnet, whose main parameters are given in Table 1. It features two nested single-layer dipole coils, the inner coil yielding a vertical dipole field and the outer coil a horizontal dipole field. The coils are individually powered with $600 \mathrm{~A}$ power supplies. The coils are wound from a $\mathrm{NbTi}$ rectangular wire bound together as a flat cable of 9 or 7 wires in the inner and

Table 1: Parameters of the LHC low- $\beta$ corrector dipole prototype, MCBX.

\begin{tabular}{|lcc|}
\hline & & Inner/Outer Coil \\
Operating dipole field & {$[\mathrm{T}]$} & $3.3 / 3.3$ \\
Integrated dipole field & {$[\mathrm{Tm}]$} & $1.341 / 1.273$ \\
Peak field in the cond. & {$[\mathrm{T}]$} & $4.431 / 4.749$ \\
Margin on the load line & & $50.51 \% / 45.54 \%$ \\
Operating current & {$[\mathrm{A}]$} & $511 / 599$ \\
Magnetic length & {$[\mathrm{m}]$} & $\mathbf{0 . 4 1 / 0 . 3 8}$ \\
Overall length & {$[\mathrm{m}]$} & 0.6 \\
Coil inner diameter & {$[\mathrm{mm}]$} & $90 / 123.7$ \\
Coil outer diameter & {$[\mathrm{mm}]$} & $119.7 / 146.8$ \\
Yoke inner diameter & {$[\mathrm{mm}]$} & 100 \\
Yoke outer diameter & {$[\mathrm{mm}]$} & 1.450 \\
Wire dimension (ins.) & {$[\mathrm{mm}]$} & $1.65 \times 0.97$ \\
Wire dimension (metal) & {$[\mathrm{mm}]$} & $1.53 \times 0.97$ \\
Cu/Sc-ratio & {$[\mathrm{K}]$} & 1.6 \\
Operating temperature & {$[\mathrm{K}]$} & 1.9 \\
No. of turns per coil & & $414 / 406$ \\
Stored energy & {$[\mathrm{kJ}]$} & $21.162 / 29.725$ \\
Self inductance & {$[\mathrm{mH}]$} & $162 / 166$ \\
\hline
\end{tabular}

the outer coil respectively, and cooled at $1.9 \mathrm{~K}$. The design field integral is $1 \mathrm{Tm}$ in any direction for an excitation current ranging from 360 to $600 \mathrm{~A}$. Due to the short length of the magnet, the end fields contribute more than $50 \%$ to the field integral. Therefore, an optimization in $2 \mathrm{D}$ and scaling with the magnetic length is not sufficient. Particular attention has to be paid to the lead end where the transitions from one block to another are made in addition to the leads entering over the top of the end blocks. Figure 1 illustrates the approach to an integrated automated design comprising the following 8 steps: 2D coil design including mathematical coil optimization, 3D coil design with mathematical coil optimization, transfer of model file to Opera- $3 \mathrm{D}^{\infty}[3]$ for calculations including the iron saturation, transfer of file to CAD for the mechanical drawings, transfer of file to the CNC-machine for machining of the end spacers.

\section{ELECTROMAGNETIC CALCULATIONS}

\subsection{Coil Optimization in $2 D$}

Based on some design constraints, which includes the space limitations and the operating current, a preliminary conductor and coil lay-out can be chosen. With only a few lines of input data the cross-section is generated by means of the ROXIE-program. ROXIE

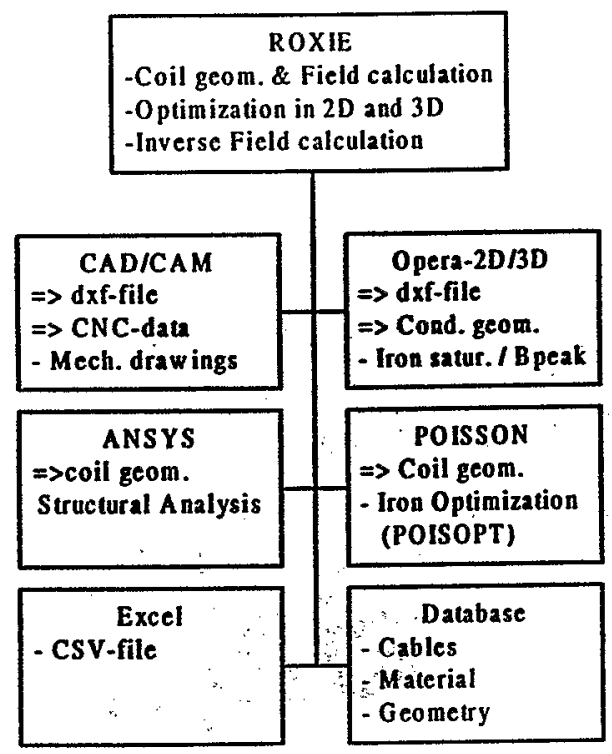

Figure : 1. Approach to an ințegrated automated magnet design 
Table $2:$ MCBX-magnet, multipole content in 2D. $(\mathrm{bi}=\mathrm{Bi} / \mathrm{B} 1$ )

\begin{tabular}{|lccc|}
\hline & $\begin{array}{c}\text { ROXIE-2D } \\
\text { linear iron }\end{array}$ & $\begin{array}{c}\text { Opera-2D }^{28} \\
\text { linear iron }\end{array}$ & $\begin{array}{c}\text { Opera-2D }^{\Phi} \\
\text { non-linear iron }\end{array}$ \\
$\mathrm{B} 1 \quad[\mathrm{~T}]$ & 3.2989 & 3.3001 & 3.2972 \\
$\mathrm{~b} 3 \times 10^{4}$ & 0.0007 & 0.0250 & -0.0446 \\
b5 $\times 10^{4}$ & 0.0580 & 0.0565 & 0.0557 \\
b7 $\times 10^{4}$ & 0.0143 & 0.0141 & 0.0141 \\
b9 $\times 10^{4}$ & 0.0001 & 0.0001 & 0.0001 \\
\hline
\end{tabular}

applies Biot Savart's law on line currents and the iron with linear or infinite permeability is taken into account by imaging. After a few manual iterations an automatic design optimization can be carried out. ROXIE includes many different optimization algorithms [2]. All the design parameters can be addressed as design variables for the optimization. One of the most robust algorithm is EXTREM, which was used for the optimization of the LHC low- $\beta$ dipole, MCBX, described here. The objectives were low peak field in the conductor and minimized multipole content.

\subsection{Optimization of the iron circuit}

Once the coil cross-section is optimized the geometry is exported in DXF-format to commercial FE-software. The Opera- $2 D^{\otimes}$ package is used to calculate the saturation effects in the iron circuit. Different inner and outer radii were investigated for variation of the harmonic content, b3 and b5 in particular, at current levels ranging from injection to $120 \%$ of the nominal current. Table 2 compares the calculated field values from ROXIE with those from Opera- $2 D^{\oplus}$ linear and nonlinear models. The main difference is the $b 3$ due to the simplified conductor blocks in the FE-model. In total 23000 isoparametric, 6-node-triangles were used. The

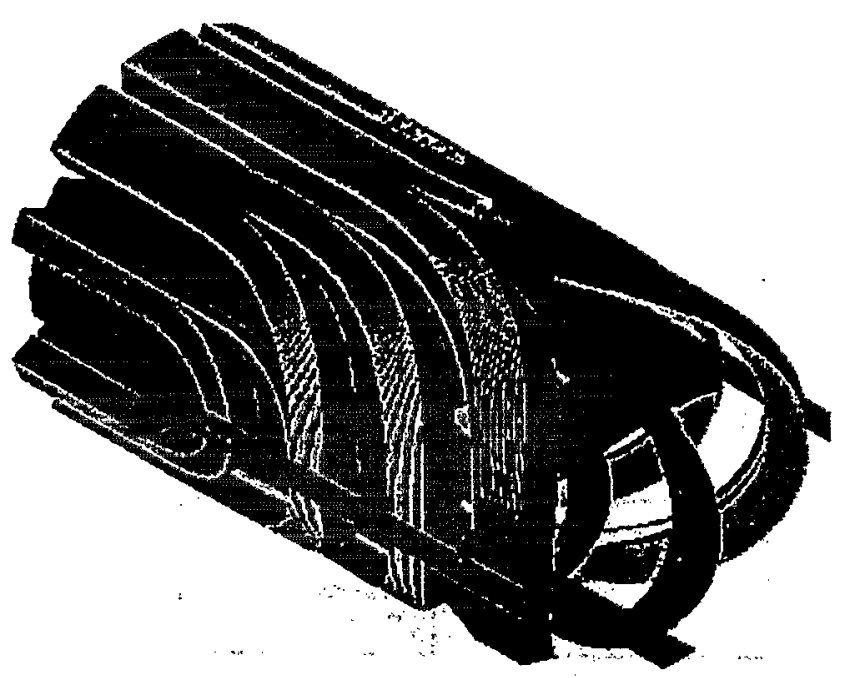

Figure : 2. MBCX-magnet lead end from ROXIE. Note the modelling of cross-over conductors
Table 3 : Integrated multipole content in 3D (bi $=\mathrm{Bi} / \mathrm{B} 1$ )

\begin{tabular}{|lccc|}
\hline & $\begin{array}{c}\text { ROXIE-3D } \\
\text { linear iron }\end{array}$ & $\begin{array}{c}\text { Opera-3D } \\
\text { linear iron }\end{array}$ & $\begin{array}{c}\text { Opera-3D } \\
\text { non-linear iron }\end{array}$ \\
$\mathrm{B} 1 \quad[\mathrm{Tm}]$ & 1.3627 & 1.3421 & 1.3423 \\
$\mathrm{~b} 3 \times 10^{4}$ & -0.3642 & 1.9654 & 1.9639 \\
b5 $\times 10^{4}$ & 0.1700 & 0.1775 & 0.1775 \\
b7 $\times 10^{4}$ & 0.0372 & 0.0381 & 0.0381 \\
b9 $\times 10^{4}$ & 0.0013 & 0.0014 & 0.0014 \\
\hline
\end{tabular}

harmonic content was evaluated at a $30 \mathrm{~mm}$ radius and scaled down to $10 \mathrm{~mm}$ radius.

\subsection{Coil Optimization in $3 D$}

Most of the quenches occur in the coil ends. Therefore, particular attention is paid to the optimization of the coil end geometry with its constant perimeter ends. In ROXIE the geometry is created with a few additional parameters: big half axis of the ellipse, angle of the cable in the yz-plane, and the axial shift in the zdirection. Before the electromagnetic optimization the end geometry is optimized to maximize the minimum radius of curvature. The user can choose from a normal ellipse or a hyper-ellipse form. Coil blocks can be aligned on the winding mandrel or on the outer radius with so-called shoes. The connection end with transitions from one block to another can be created with an asymmetric model. The leads entering from outside are modeled as 8-node-bricks from pre-defined cut planes. The field calculation in 3D cannot take into account the iron except for the integrated multipole content, when the calculation is carried out from the symmetry plane far enough along the $z$-axis. The field optimization is done by varying the axial position of the blocks.

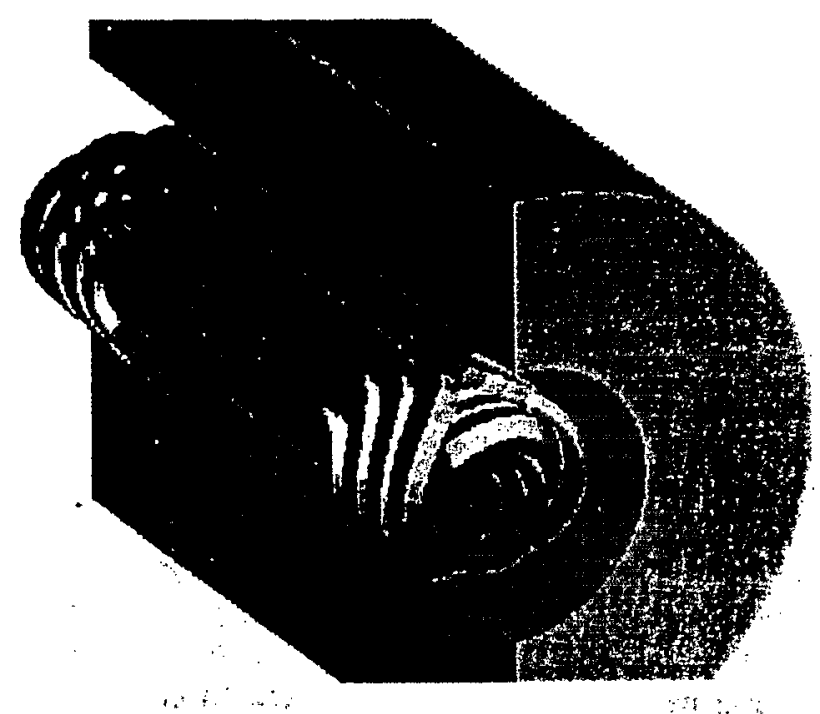

Figure : $3 . \mathrm{MBCX}$-magnet Opera-3D model. Only the return ends modelled for simplicity. 

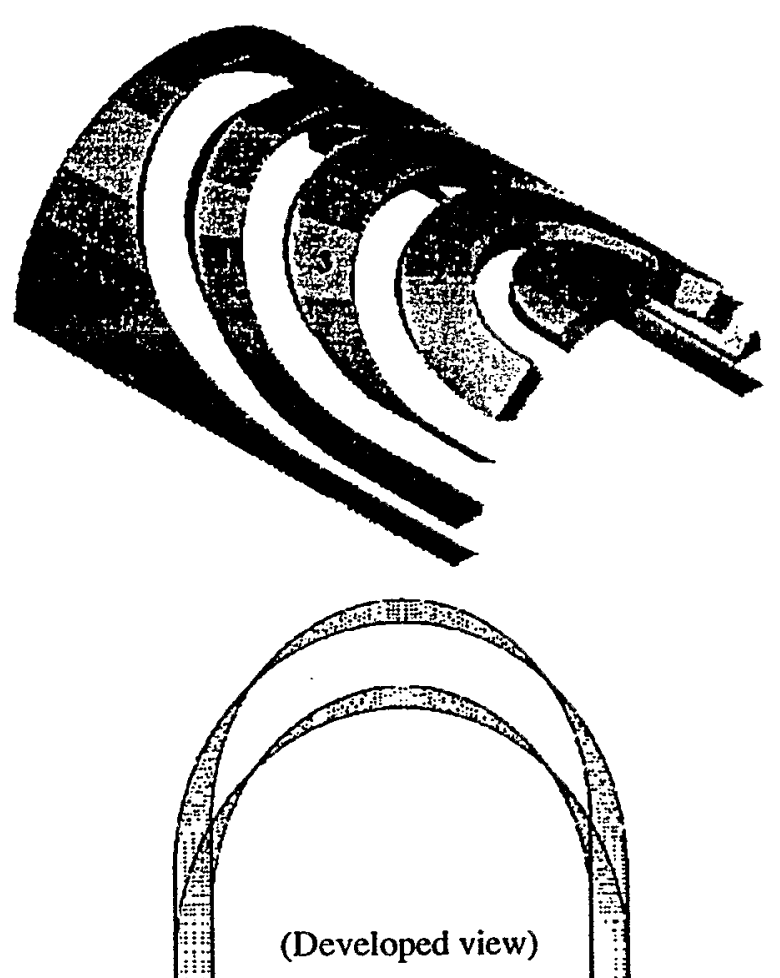

Figure : 4. End-spacers and the 3D-polygons for CNCmachining of spacer no. 3 .

\subsection{FE-analysis in $3 D$}

The influence of the non-linear iron on the field quality and the peak fields is studied with Opera-3D ${ }^{\oplus}$. The optimized conductor geometry can be imported from ROXIE as 8-node-bricks or as 20-node-bricks. This speeds up considerably the modelling time, since these geometries cannot be created in Opera using the built-in constant-perimeter-end coil primitive. Reduced scalar potential was used for all the regions inside the iron. Table 3 compares the integrated multipole content of ROXIE with linear iron $\left(\mu_{\mathrm{r}}=2000\right)$ to that of the Opera$3 D^{\infty}$ model. A set of dummy conductors, which are duplicates of the 'active' ones without imaged parts, and with zero current, were modelled to speed up the peakfield calculation. Only the part of the geometry, which was solved in Tosca ${ }^{\circledR}$ had to be activated. A considerable enhancement of the peak field was found in 3D: $4.75 \mathrm{~T}$ with respect to $4.23 \mathrm{~T}$ in $2 \mathrm{D}$.

\section{MECHANICAL DESIGN}

\subsection{Structural analysis}

Microscopic movements of the conductors or microcracking of epoxy can dissipate locally enough energy to cause a super conducting magnet to quench. Therefore a sufficient coil pre-compression is essential for the magnet performance. Too high pre-stress, however, can damage the insulation or cause copper to yield. The
ROXIE conductor geometry was imported to ANSYS $^{\circledR}$ [5]. An electromagnetic model was first created to determine the Lorenz forces, which were then transferred to the mechanical model. Five load steps were considered: assembly at $293 \mathrm{~K}$, cooldown to $1.9 \mathrm{~K}$, inner or outer coil powered, and the combined field.

\subsection{CAD-CAM}

The coil cross-section, developed view (sz-plane) and cut through the end (yz-plane) can be exported from ROXIE to most CAD programs in DXF format. The end spacer geometry is defined by 9 polygons, presented in global xyz-coordinates, over the radius and can be imported to CAD and CAM packages. These rather complicated shapes are then machined with a 5-axis CNC machine.

\subsection{Mechanical tolerances}

The sensitivity analysis for the mechanical tolerances is carried out in ROXIE by giving the tolerances as upper and lower bounds for the number of geometrical design variables. The upper and lower quotient per unit displacement are then calculated, and the Jacobian error matrix can be taken into a spreadsheet program for further processing.

\section{CONCLUSIONS}

A number of tools to speed up the design process and decision making have been established in the ROXIE program as an approach towards an integrated design of superconducting magnets. The optimized conductor geometry can be directly taken into commercially available FE-programs. Links to CAD/CAM-packages allow creation and changes of the mechanical drawings to be done in parallel with the design optimization. Short correction magnets, which in addition to optimization require studying of the manufacturing tolerances in 3D, can be efficiently designed by these means.

\section{REFERENCES}

[1] 'A Computer Program for the Design of Superconducting Accelerator Magnets', by $S$. Russenschuck, Proceedings of ACES'95, Monterey, CA, USA, March 20-24, 1995, LHC Note 354, Geneva, Switzerland, 1995

[2] 'Synthesis, Inverse Problems and Optimization in Computational Electromagnetics', by $S$. Russenschuck, International Journal of Numerical Modeling: Electronic Network Devices and Fields, Vol. 45-57 (1996).

[3] Opera-2D ${ }^{\oplus}$, Opera-3D $D^{\circledast}$ and Tosca ${ }^{\oplus}$, Trademarks from Vector Fields Limited, Oxford, England

[5] ANSYS $^{\oplus}$, Trademark from SWANSON Analysis Inc., Houston, PA, USA 


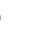




\title{
PROGRESS IN THE DEVELOPMENT OF THE 1-m MODEL OF THE $70 \mathrm{~mm}$ APERTURE QUADRUPOLE FOR THE LHC LOW- $\beta$ INSERTIONS
}

\author{
S.R. Milward, S. Nobes, K.D. Smith, A.J. Street, M.C. Townsend, \\ J.R. Treadgold, J.M. Wiatrzyk, Oxford Instruments, Oxfordshire, England, \\ G.A. Kirby, R. Ostojic, J. Strait, * T.M. Taylor, CERN, Geneva, Switzerland
}

\section{Abstract}

Within the LHC magnet development program Oxford Instruments has built a one metre model of the $70 \mathrm{~mm}$ aperture low- $\beta$ quadrupole. The magnet features a four layer coil wound from two $8.2 \mathrm{~mm}$ wide graded $\mathrm{NbTi}$ cables, and is designed for $250 \mathrm{~T} / \mathrm{m}$ at $1.9 \mathrm{~K}$. The magnet has previously been tested between $4.5 \mathrm{~K}$ and $2.3 \mathrm{~K}$. In this paper we review the magnet rebuild and the subsequent tests. Results on magnet training at $4.3 \mathrm{~K}$ and $1.9 \mathrm{~K}$ are presented along with the results related to quench protection studies.

\section{INTRODUCTION}

As part of the LHC magnet development program Oxford Instruments has built and tested a one metre model of the $70 \mathrm{~mm}$ aperture quadrupole for the low- $\beta$ insertions. The design and construction of this magnet has been reported previously along with the results of the first tests $[1,2]$. The magnet [3] has a graded 4-layer coil with the transition between the two cable types in the middle of the 2nd layer. Thin collars $10 \mathrm{~mm}$ wide ensure accurate coil location during assembly. Final preload is applied using a set of aluminum collet force rings, whose load is transmitted to the collared coil through the 4-piece iron yoke.

During the first tests in March 1995, the magnet reached a maximum current of $3780 \mathrm{~A}$ at $4.5 \mathrm{~K}$ and of $4112 \mathrm{~A}$ at $2.5 \mathrm{~K}$. After this point the performance became erratic and it was not possible to train further. It was thought that the quenches were caused by conductor motion. The magnet was warmed to room temperature and the coil preload increased by tightening the force rings. The magnet was tested again in August 1995, but repeatedly quenched at currents between 3045 and $3180 \mathrm{~A}$. The quench current was independent of temperature. The quenches were all in layers 3 and 4 of quadrant $D$, but the instrumentation was insufficient to locate the quenches within this coil pair. The magnet had protection resistors fitted across each pole which prevented further diagnostic testing without removal of the magnet from the test dewar.

In this paper we describe the rebuild of the magnet and present the results of the subsequent tests. The magnet has been energised to a gradient above the LHC operating point and measurements have been made of the quench velocities and peak temperatures.

\footnotetext{
- Permanent address: Fermilab, Batavia, Hlinois, USA
}

\section{DISASSEMBLY AND REBUILD}

With the magnet at room temperature and the support structure disconnected, the protection resistors across each pole were removed and the external joints broken. Measurements of resistance and inductance for each coil indicated the presence of a multi-turn short in quadrant $D$. After the disassembly of the magnet, the location of the short was determined by applying a current to the coil and measuring the voltage to each turn. A multi-turn shortcaused by scissoring was found in the ramp between layers 3 and 4 . About half the strands of one piece of cable were damaged. In hindsight, this region was probably first damaged during energisation to 4112 A in March 1995.

The size and location of the short precluded any attempts at repair and a replacement coil was wound with modified geometry in the layer ramp region. This modification was also made to all the other coils to prevent similar damage. Due to availability of material, the rewound coil incorporated a modified insulation system which was $9 \mu \mathrm{m}$ thicker per turn than in the other coils. To compensate for the increased thickness the sizes of the copper wedges at the pole were reduced. The magnet was rebuilt with a modified set of voltage taps on all coils and all the ground plane insulation replaced. The coil prestress at room temperature was reset to the same value as previously. In all other ways the magnet was identical to the first build.

\section{MAGNET TESTS}

The magnet was equipped with a set of spot heaters located between turns, one in each of the inner three layers of each quadrant. The heaters could be energised individually to trigger a quench, and all were energised once a quench was detected. Bypass resistors of $R=2.3 \mathrm{~m} \Omega$ were connected across each quadrant of the coil in an effort to provide additional protection. Although complicating analysis, these resistors were refitted, since the previous tests had not produced sufficient data to allow confidence in their removal.

\subsection{Training History}

The training history is displayed in Fig. 1. This figure also shows the estimated conductor limits (2-4\% uncertainty), the calculated gradients (including iron saturation) and the operating gradients $G_{o p}$ of two versions of this magnet. The high luminosity low $-\beta$ insertions utilize a single aperture quadrupole (MQX), operated at $\mathrm{G}_{\mathrm{op}}=225 \mathrm{~T} / \mathrm{m}$ and $1.9 \mathrm{~K}$. 


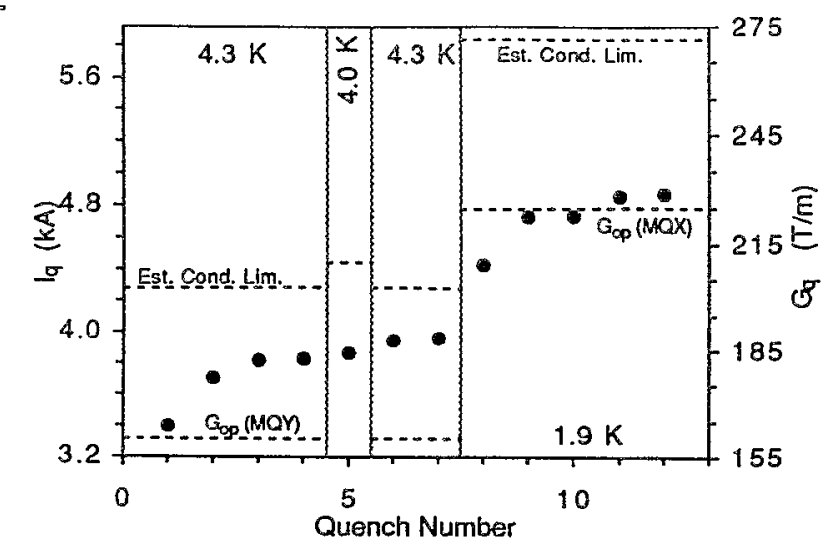

Figure 1: Quench training history.

In the dump insertion, a two-in-one version (MQY) operating at $4.5 \mathrm{~K}$ with $\mathrm{G}_{\mathrm{op}}=160 \mathrm{~T} / \mathrm{m}$ is envisaged.

The magnet was initially trained at $4.3 \mathrm{~K}$ and the first quench occurred at $3408 \mathrm{~A}$. This was about $150 \mathrm{~A}$ lower than the first quench of the first test. The next three quenches were at $3712 \mathrm{~A}, 3828 \mathrm{~A}$ and $3833 \mathrm{~A}$, slightly above the plateau of the first test. In view of possible conductor limitation at $4.3 \mathrm{~K}$, the magnet was pumped to $4.0 \mathrm{~K}$ and energised with a quench at $3860 \mathrm{~A}$. The increase of $30 \mathrm{~A}$ was less than the expected gain by cooling and further training was continued at $4.3 \mathrm{~K}$. The following quenches at $3953 \mathrm{~A}$ and 3958 A were deemed to represent a relatively stable plateau, and the magnet was cooled to superfluid helium temperature. Throughout this stage quenches occurred in all the coils except in the rebuilt coil D, although coil C (layers 2 and 4) showed a propensity for quenching.

The first quench in superfluid helium occurred at $4441 \mathrm{~A}$. The next two quenches were recorded at $4746 \mathrm{~A}$ and $4743 \mathrm{~A}$, with a change in quench location. On the next two runs the current increased to $4862 \mathrm{~A}$ and $4879 \mathrm{~A}$. Further training was discontinued due to test equipment failure.

At both test temperatures, the magnet has achieved a plateau above the operating gradients required for the LHC. However, the plateaus are achieved somewhat below the computed conductor limits. The last quench at each temperature was in layer 2, where conductor limited quenches are expected to occur, but the instrumentation is insufficient to determine if these quenches occur at the high field point. The coil pressure is measured to be $74 \mathrm{MPa}$ at zero field and decreases by only $13.3 \mathrm{MPa}$ up to a current of $3750 \mathrm{~A}$, suggesting that the coil remains adequately preloaded to well above the highest quench current achieved. Further testing will be required to verify if the magnet is mechanically limited or if the actual conductor limit is lower than that computed.

\subsection{Quench Protection Studies}

An important objective of this test was to measure the dependence of the peak conductor temperature $T_{\text {peak }}$ and the initial quench velocity $v_{q}$ on the magnet current $\mathrm{I}_{\mathrm{q}}$, in or- der to help in the design of a quench protection system for a full-length magnet.

For these studies, special voltage taps were used. They are placed adjacent to the spot heater (HA2), which is located near the lead end of the second layer of quadrant $A$ between turns of the small cross-section cable. By measuring the final resistance of the $125 \mathrm{~mm}$ long cable segment which contains HA2, $T_{\text {peak }}$ can be determined. Similarly, by measuring the time necessary for the quench front to propagate to another tap $585 \mathrm{~mm}$ away $v_{q}$ can be determined. The quench velocity can be independently measured from the initial resistance growth $\mathrm{dR} / \mathrm{dt}$ and the measured resistance per unit length of the cable (including magneto-resistance). The measured $\int \mathrm{I}^{2} \mathrm{dt}$ can be converted to peak temperature using the known conductor properties and the magnetic field at the point of the quench.

Figure 2 shows the $v_{\mathrm{q}}$ measured by time of flight $(\Delta \mathrm{t})$ versus $I_{q}$. For spontaneous quenches which do not originate in quadrant $\mathrm{A}$, the quench at $\mathrm{HA} 2$ is still triggered by that heater and $v_{\mathrm{q}}$ can be determined. Spontaneous and heaterinduced quenches are displayed independently in Fig. 2; they clearly display the same trend. The $v_{q}$ grows from about $5 \mathrm{~m} / \mathrm{s}$ at low current to $65 \mathrm{~m} / \mathrm{s}$ for the highest current $4.3 \mathrm{~K}$ quench. At $1.9 \mathrm{~K}, \mathrm{v}_{\mathrm{q}}$ grows more slowly with current, but quenches at a similar fraction of the conductor limit at the two temperatures have similar velocities.

The quench velocity can also be measured from the initial dR/dt. Comparison is made between the velocities determined by the two methods in Fig. 3. For $v_{\mathrm{q}} \leq 20 \mathrm{~m} / \mathrm{s}$ the two methods agree well; however for larger $v_{\mathrm{q}}$ the $\mathrm{dR} / \mathrm{dt}$ method gives a systematically lower value. Since $\mathrm{dR} / \mathrm{dt}$ also has a component due to resistivity growth with temperature, the velocity measurements are based only on the first $6-8 \mathrm{~ms}$ after the quench enters the cable segment adjacent to HA2. The $\Delta t$ method, on the other hand, the velocity averaged over the time the quench front propagates between the two taps at the end of the $585 \mathrm{~mm}$ segment adjacent to HA2. For two quenches, with $\Delta t$ velocities of 20 and $45 \mathrm{~m} / \mathrm{s}, v_{\mathrm{q}}$ ob-

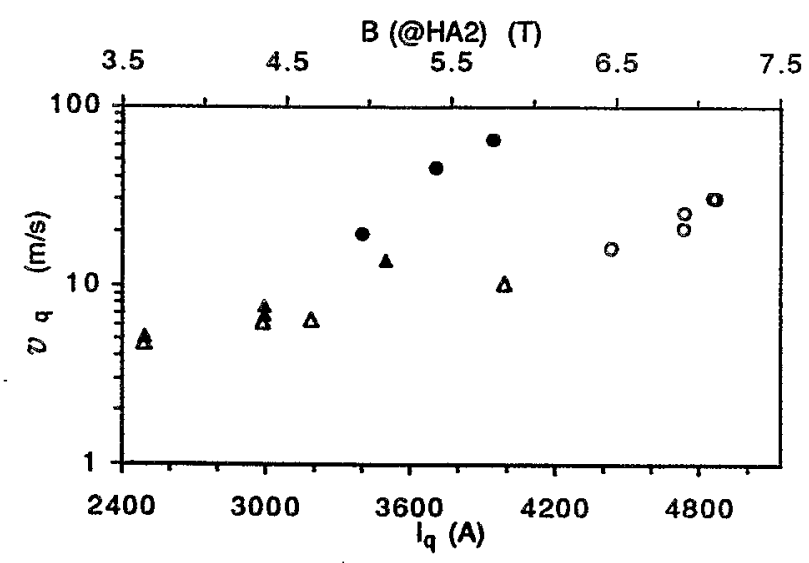

Figure 2: $v_{q}$ vs. $\mathrm{I}_{\mathrm{q}}$ for heater $\mathrm{HA} 2$ induced (triangles) and spontaneous (circles) quenches. Quenches at 4.3 (1.9) K are shown as filled (open) symbols. 


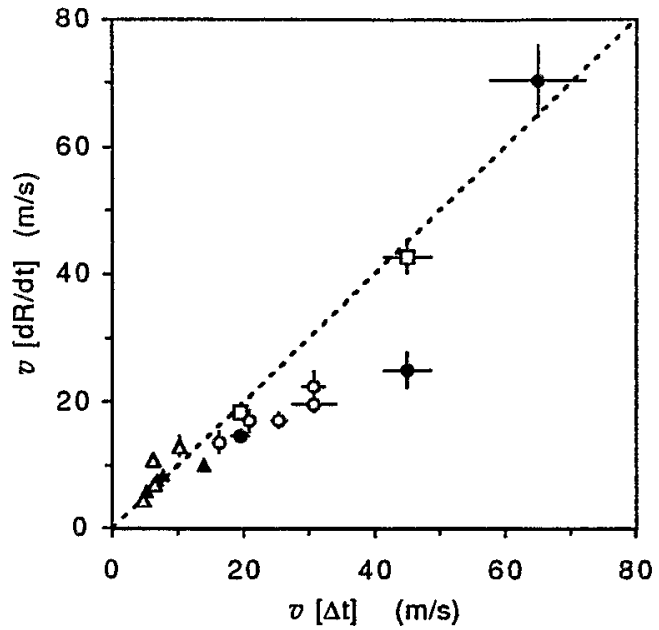

Figure 3: $v_{\mathrm{q}}$ measured by initial $\mathrm{dR} / \mathrm{dt}$ vs that by $\Delta \mathrm{t}$ for heater HA2 induced (triangles) and natural(circles) quenches. Quenches at 4.3 (1.9) $\mathrm{K}$ are shown as filled (open) symbols. The open squares give the $v_{q}$ measured by average $\mathrm{dR} / \mathrm{dt}$ for the same quenches plotted directly below them.

tained on the basis of the average $\mathrm{dR} / \mathrm{dt}$ were calculated, and are shown in Fig. 3. For the highest $v_{\mathrm{q}}$ point $\Delta \mathrm{t}$ is too short to measure initial and average $\mathrm{dR} / \mathrm{dt}$ independently and the two calculations coincide. The velocities obtained from the average $\mathrm{dR} / \mathrm{dtagree}$ at both low and high $v_{\mathrm{q}}$ with those from the $\Delta t$ method. This strongly suggests there is an acceleration of the quench front, at least during the time interval covered by these data.

Figure 4 displays $T_{\text {peak }}$ vs. $I_{q}$ for heater induced quenches at two operating temperatures. At $4.3 \mathrm{~K}$, $\mathrm{T}_{\text {peak }}$ has a maximum of about $250 \mathrm{~K}$ which occurs between 3000 and $3500 \mathrm{~A}, 70-80 \%$ of the estimated conductor limit. $T_{\text {peak }}$ is substantially higher at $1.9 \mathrm{~K}$ and no maximum is evident up to $4000 \mathrm{~A}$. However, if the highest temperature occurs at $70-80 \%$ of the conductor limit, the $4000 \mathrm{~A}$ point is close to the maximum. Based on this consideration, it was deemed safe to train the magnet at $1.9 \mathrm{~K}$.

To estimate $T_{\text {peak }}$ for spontaneous quenches, $\int \mathrm{I}^{2} \mathrm{dt}$ is computed and displayed in Fig. 5 for the heater induced and the spontaneous quenches. For the heater quenches, the pattern of $\int I^{2} d t$ and $T_{\text {peak }} v I_{q}$ are similar, as expected. The induced and natural quenches follow similar trend in $\int \mathrm{I}^{2} \mathrm{dt}$ vs $\mathrm{I}_{\mathrm{q}}$. At $1.9 \mathrm{~K}, \int \mathrm{I}^{2} \mathrm{dt}$ achieves a maximum near $4400 \mathrm{~A}$, about $75 \%$ of the conductor limit, and decreases substantially at higher current. The $T_{\text {peak }}$ for the highest point is computed to be $465 \pm 40 \mathrm{~K}$, where the error includes uncertainties in the start time of the integration and in the value of $B$ at the quench location.

The coil resistance $R(t)$ as a function of time and the energy deposited in the coil $\int \mathrm{I}^{2} \mathrm{Rdt}$ were computed. It was found that only about $0.1 \%$ of the stored energy was deposited in the bypass resistors, the rest being absorbed by the coil. Since the magnet absorbs its own energy safely, the protection resistors can be removed for subsequent tests.

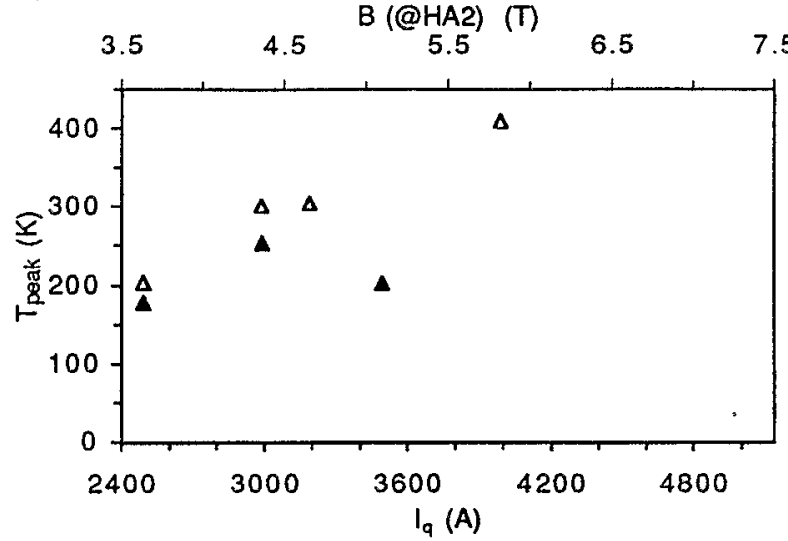

Figure 4: $T_{\text {peak }}$ vs. $I_{q}$ for quenches induced by HA2. Quenches at 4.3 (1.9) K are shown as filled (open) symbols.

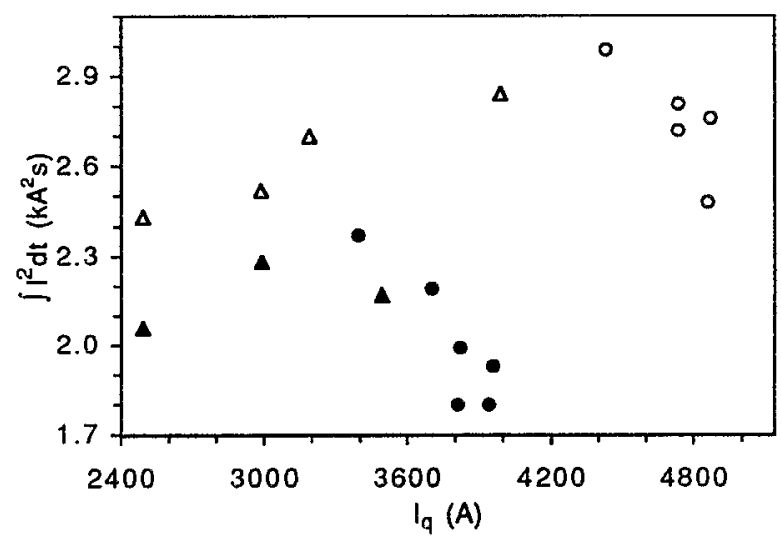

Figure 5: $\int \mathrm{I}^{2} \mathrm{dt}$ vs. $\mathrm{I}_{\mathrm{q}}$ for spontaneous (circles) and heater HA2 induced (triangles) quenches. Quenches at 4.3 (1.9) K are shown as filled (open) symbols.

\section{CONCLUSIONS AND FUTURE PLANS}

The one metre model of the $70 \mathrm{~mm}$ aperture quadrupole for the LHC low- $\beta$ insertions built by Oxford Instruments has achieved the operating current of $4790 \mathrm{~A}(225 \mathrm{~T} / \mathrm{m})$ with only three training quenches in superfluid helium. In comparison with other quadrupoles tested for the LHC, the highest gradient times aperture has been achieved. The mechanical structure of the magnet was shown to be appropriate, and the construction was achieved without the use of expensive tooling. A further test of the magnet is planned in order to measure the field quality, examine the training behaviour following a thermal cycle, and collect additional data relevant for quench protection.

\section{REFERENCES}

[1] S. Nobes et al., Submitted to the 14th Int. Conf. on Magnet Technology, Tampere, Finland, June 1995.

[2] G. A. Kirby et al., Proc. 4th European Particle Accelerator Conference, London, England, June 1994, pp 2271-2273

[3] R Ostojic, T.M. Taylor and G.A. Kirby, IEEE Trans. Magn. 30 pp 1750-1753, 1994. 



\title{
OPTIMIZATION OF THE LHC INTERACTION REGION WITH RESPECT TO BEAM-INDUCED ENERGY DEPOSITION
}

\author{
N. Mokhov, Fermilab, Batavia, Illinois, USA \\ J. Strait, * CERN, Geneva, Switzerland
}

\begin{abstract}
Energy deposition in the superconducting magnets by particles from p-p collisions is a significant challenge for the design of the LHC high luminosity insertions. We have studied the dependence of the energy deposition on the apertures and strengths of insertion magnets and on the placement of absorbers in front of and within the quadrupoles. Monte Carlo simulations were made using the code DTUJET to generate $7 \times 7 \mathrm{TeV}$ p-p events and the code MARS to follow hadronic and electromagnetic cascades induced in the insertion components. The 3D geometry and magnetic field descriptions of the LHC-4.1 lattice were used. With a quadrupole coil aperture $\geq 70 \mathrm{~mm}$, absorbers can be placed within the magnet bore which reduce the peak power density, at full luminosity, below $0.5 \mathrm{~mW} / \mathrm{g}$, a level that should allow the magnets to operate at their design field. The total heat load can be removed by a cooling system similar to that used in the main magnets.
\end{abstract}

\section{INTRODUCTION}

The Large Hadron Collider (LHC) [1] is designed to produce p-p collisions at $\sqrt{s}=14 \mathrm{TeV}$ and $L=10^{34} \mathrm{~cm}^{-2} \mathrm{~s}^{-1}$. The interaction rate of $8 \times 10^{8} \mathrm{~s}^{-1}$ represents a power of almost $900 \mathrm{~W}$ per beam, the large majority of which is directed towards the low- $\beta$ insertions. Previous studies [2, $3,4]$ have identified this as a potentially serious problem. The quadrupole fields sweep the secondary particles into the coils preferentially along the vertical and horizontal planes, giving rise to local peak power density $P_{\max }$ as much as an order of magnitude larger than the average. Tests of porous cable insulation systems [5] cooled by a $1.9 \mathrm{~K}$ helium bath have shown that for typical cable dimensions up to about $1 \mathrm{~mW} / \mathrm{g}$ of heat can be removed while keeping the helium inside the cable below $2.2 \mathrm{~K}$. This is the most important point of this study, since too large $P_{\max }$ could prevent the low- $\beta$ quadrupoles from reaching their required gradient.

To minimize $P_{m a x}$, we have studied its dependence on the aperture of the front absorber and of absorbers placed inside the magnets, the low- $\beta$ quadrupole coil diameters, and the beam separation/recombination dipole length. Solutions which satisfy the requirements with a reasonable safety margin have been found.

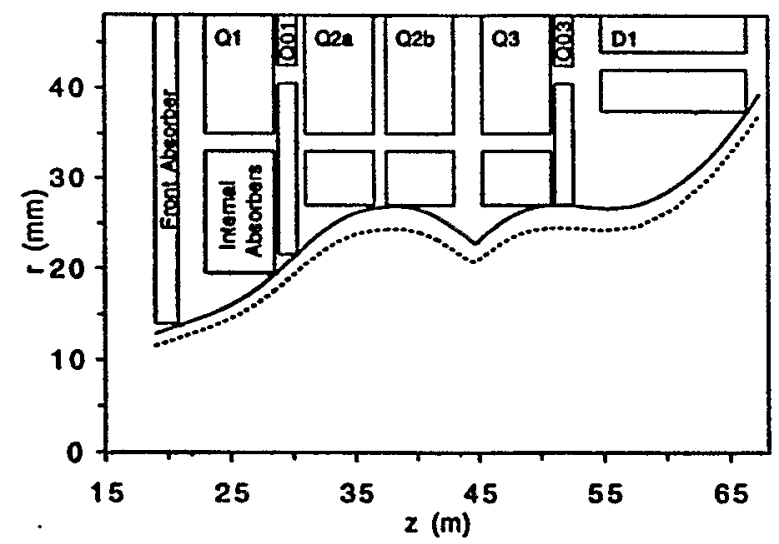

Figure 1: The LHC low- $\beta$ insertion including absorbers which reach the $10 \sigma$ limit (injection/collision optics: solid/dashed line) for $d_{\text {coil }}=70 \mathrm{~mm}$ quadrupoles.

\section{COMPUTER MODELLING}

Fig. 1 shows the LHC low- $\beta$ insertion $[1,6]$. The inner triplet is made of four identical high-gradient quadrupoles with coil inner diameter $d_{\text {coil }}=70 \mathrm{~mm}[7](\mathrm{Q} 1$ and $\mathrm{Q} 3$ focusing and Q2a and Q2b defocusing), which are powered in series and operate at a maximum gradient of $227 \mathrm{~T} / \mathrm{m}$ at the high luminosity IRs. Two independently powered "trim" quadrupoles of $d_{\text {coil }}=85 \mathrm{~mm}$ and maximum operating gradient of $120 \mathrm{~T} / \mathrm{m}$ (Q01 and $\mathrm{Q} 03$ ) provide the additional strength required by $Q 1$ and $Q 3$ and allow tuning of the triplet. Behind the triplet are the dipoles D1 (single aperture) and D2 (twin aperture). They have $d_{\text {coil }}=85 \mathrm{~mm}$ and an operating field of $4.3 \mathrm{~T}$. Their length $(11.5 \mathrm{~m})$ is set by the required strength at the combined experimental and injection insertions (points 2 and 8), where space is more limited than at the high luminosity IRs (points 1 and 5).

Alternate IR designs with quadrupoles of $d_{\text {coil }}=60 \mathrm{~mm}$ and $80 \mathrm{~mm}$ have also been considered. The gradients were scaled with $d_{\text {coil }}$ (a little more slowly than $1 / \mathrm{r}$ for thick shell quadrupoles) and corresponding length changes were made. Optics with minimal perturbations to the baseline were computed for injection ( $450 \mathrm{GeV}, \beta^{*}=6 \mathrm{~m}$ ) and collision ( $7 \mathrm{TeV}$, $\beta^{*}=0.5 \mathrm{~m}$ ) conditions and are summarized in Table 1. Relative to the baseline $70 \mathrm{~mm}$ case $\beta_{\max }$ for $80(60) \mathrm{mm}$ quadrupoles changes by $+6.6 \%(-5.1 \%)$ yielding changes in the maximum beam size of $+3.3 \%(-2.6 \%)$, considerably less than the change in $d_{\text {coil }}$. Thus increasing the aperture should improve the field quality over the region occupied by the beam and allow more shielding inside the magnet bore, 
Table 1: Characteristics of the IR optics.

\begin{tabular}{|c|c|c|c|c|c|c|}
\hline $\begin{array}{l}d_{\text {coil }} \\
L_{\text {mag }}\end{array}$ & \multicolumn{2}{|c|}{$\begin{array}{c}60 \mathrm{~mm} \\
5.1 \mathrm{~m}\end{array}$} & \multicolumn{2}{|c|}{$\begin{array}{c}70 \mathrm{~mm} \\
5.5 \mathrm{~m}\end{array}$} & \multicolumn{2}{|c|}{$\begin{array}{c}80 \mathrm{~mm} \\
6.0 \mathrm{~m}\end{array}$} \\
\hline Stage & Coll. & Inj. & Coll. & Inj. & Coll. & $\overline{\text { Inj }}$ \\
\hline $\mathrm{G}(\mathrm{T} / \mathrm{m}$ & & & & & & \\
\hline & 251 & & 227 & 14.0 & 202 & 12.4 \\
\hline & 70 & & 80 & 5.3 & 92 & 6.1 \\
\hline & 105 & & 101 & 7.7 & 95 & 6.1 \\
\hline$\beta_{\max }(\mathrm{m})$ & 4204 & 358 & 4431 & 377 & 4724 & 402 \\
\hline
\end{tabular}

Table 2: Minimum inner radii of absorbers.

\begin{tabular}{|l||r|rr|r|}
\hline \multicolumn{1}{|l||}{$d_{\text {coil }}$} & $60 \mathrm{~mm}$ & \multicolumn{2}{|c|}{$70 \mathrm{~mm}$} & $80 \mathrm{~mm}$ \\
Clearance & $10 \sigma$ & $10 \sigma$ & $8 \sigma$ & $10 \sigma$ \\
\hline \hline Collimator & 14.0 & 14.0 & 12.0 & 14.0 \\
Q1 & 19.0 & 19.5 & 17.0 & 20.0 \\
Q01 & 21.5 & 21.5 & 18.5 & 22.0 \\
Q2-Q03 & 26.5 & 27.0 & 23.5 & 28.0 \\
D1 & 36.5 & 37.5 & 34.0 & 38.0 \\
\hline
\end{tabular}

while decreasing the aperture will have the opposite effect.

A $1.8 \mathrm{~m}$ long copper absorber is placed in front of the triplet and stainless steel absorbers are placed within the magnet bores to minimize the energy deposition in the coils. The LHC design requires [1] that the physical aperture, including effects of dispersion, closed orbit errors, construction and alignment tolerances, and the crossing angle in the IRs, be everywhere at least $10 \sigma$ (except at the beam cleaning collimators), where $\sigma$ is the rms beam size. Fig. 1 shows the $10 \sigma$ limit for injection and collision conditions and absorbers with inner radius $r_{\text {in }}$ at this limit. The cusp between $\mathrm{Q} 2 \mathrm{~b}$ and Q3 is where the maximum $\beta$ changes from one plane to the other. The outer radius of the internal absorbers is $2 \mathrm{~mm}$ less than $r_{\text {coil }}$. Table 2 gives $r_{\text {in }}$ of the absorbers for the three quadrupole diameters. To allow the effectiveness of the absorber to be evaluated versus thickness for a fixed insertion design, the $r_{\text {in }}$ for $8 \sigma$ are given for the $70 \mathrm{~mm}$ quadrupoles. As the $r_{\text {coil }}$ grows from 30 to $40 \mathrm{~mm}$ the $10 \sigma$ limits increase by only $0.5 \mathrm{~mm}(\mathrm{Q} 1-\mathrm{Q} 1)$ and $1.5 \mathrm{~mm}(\mathrm{Q} 2-$ Q03), allowing an increased absorber thickness. However, the Dl absorber decreases from 5.5 to $4 \mathrm{~mm}$ thick.

The p-p collisions and showers in the IR components are simulated with the DTUJET93 event generator [8] and the MARS code [9], version 13(96) respectively. Charged particles are tracked through the lattice and the fields within each magnetic element. The cut-off energies are $1 \mathrm{MeV}$ (charged particles), $0.2 \mathrm{MeV}$ (photons) and 0.5 eV (neutrons). Magnet coils are modeled with 4 radial bins of $8.5 \mathrm{~mm}$ depth, azimuthal bins varying from $5^{\circ}$ at the horizontal and vertical planes to $15^{\circ}$ between, and axial bins between $1.1 \mathrm{~m}(\mathrm{Q} 1)$ and $3.8 \mathrm{~m}$ (D1) long. The magnet coils; which are a mixture of $\mathrm{NbTi}$, copper, insulation and helium, are simulated as a homogeneous material with $A=50, Z=23$ and $\rho=7, g / \mathrm{cm}^{3}$. Details such as cooling channels in the yoke and coil ends are not included. Statistical errors on the Monte Carlo calculation are estimated to be $\pm 15 \%$ for $P_{\max }, \pm 6 \%$ for the energy deposited in each magnet, and $\pm 1 \%$ for the total power in the inner triplet, based on comparison of results from different runs with independent random seeds.

\section{RESULTS -}

Fig. 2 shows $P_{\max } v s z$ for the IR with $70 \mathrm{~mm}$ quadrupoles and no internal absorbers, absorbers at $10 \sigma$ and $8 \sigma$, and all quadrupole absorbers of a uniform radius at the $10 \sigma$ limit in Q2-Q03. The front absorber aperture is set at $10 \sigma$ for the case of no internal absorbers. With no internal absorber $P_{\max }=1.2 \pm 0.2 \mathrm{~mW} / \mathrm{g}$, at or above the allowable limit. With individually sized $10 \sigma$ absorbers the peak is a factor of 3 smaller, giving a reasonable safety margin. Use of an $8 \sigma$ absorber reduces $P_{\max }$ in $\mathrm{Q} 1$, but there is little overall improvement. Increasing $r_{\text {in }}$ of the absorbers in Q1-Q01 to match the other quadrupoles results in a $25 \%$ increase in $P_{\max }$. However, this increase may not be statistically significant and further study will be required to determine if it is necessary to use different absorbers in $Q 1$ and $Q 01$ than in the rest of the triplet.

An unacceptably large $P_{m a x}$ is observed at the back of D1 even with a $10 \sigma$ absorber. However, at the high luminosity IRs it is possible to move the outer dipole D2 up to an additional $90 \mathrm{~m}$ farther from D1. This would reduce the length of D1 to one-third its present value, corresponding to the first bin in Fig. 2, which has an acceptable power density Alternatively the integrated strength would be low enough to allow use of conventional magnets for D1 and D2, eliminating the problem altogether.

The cases of three quadrupole diameters with $10 \sigma$ absorbers are compared in Fig. 3. $P_{\max }$ is $40 \%$ larger (30\% smaller) with $60 \mathrm{~mm}(80 \mathrm{~mm})$ quadrupoles than the baseline $70 \mathrm{~mm}$ case. The reduced margin with $d_{\text {coil }}=60 \mathrm{~mm}$ makes this option unattractive. The $80 \mathrm{~mm}$ case has a significantly larger margin, which could be used, if required, to provide additional physical aperture. The larger $\beta_{\max }$ is unlikely to be a problem since the field quality in the region occupied by the beam would be better with a larger aperture magnet.

Shown also is the case in which all quadrupoles, including $Q 01$ and $Q 03$, have the same $d_{\text {coil }}=70 \mathrm{~mm}$ and the $a b$ sorbers have a uniform $r_{\text {in }} . P_{\text {max }}$ is $80 \%$ larger than the case with $85 \mathrm{~mm}$ trims and individually sized absorbers (Fig. 3) and $45 \%$ larger than with uniform absorbers (Fig. 2). A parently it is unacceptable to have a continuous annular $g$ : between the absorber outer and coil inner radii.

Table 3 summarizes the total power deposited in the $m$ nets and internal absorbers. The quadrupoles and the dif: D1 are considered separately, since the actual dipole c figuration will probably be different than that consid here. There is little difference among the cases with . ternal absorbers. Up to half the power is deposited in the absorbers, and it is tempting to consider cooling them at a higher temperature. However, the insulating space between the absorber and the vacuum pipe would reduce the absorber 


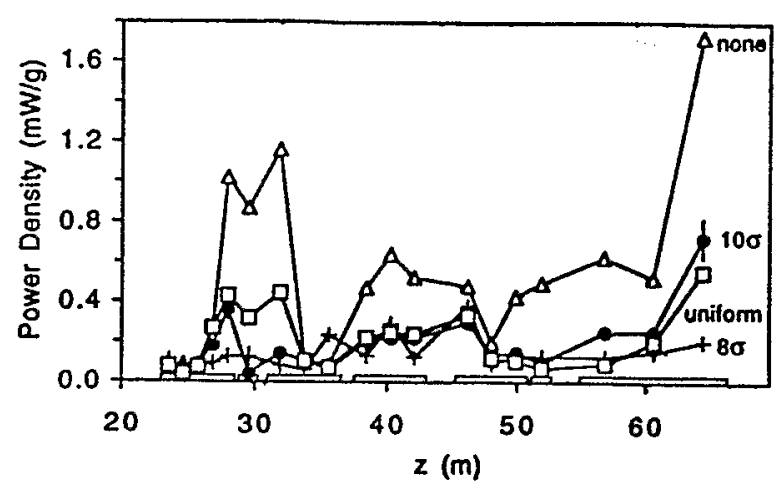

Figure 2: $P_{\max }$ vs $z$ for $70 \mathrm{~mm}$ quadrupoles with several absorber configurations.

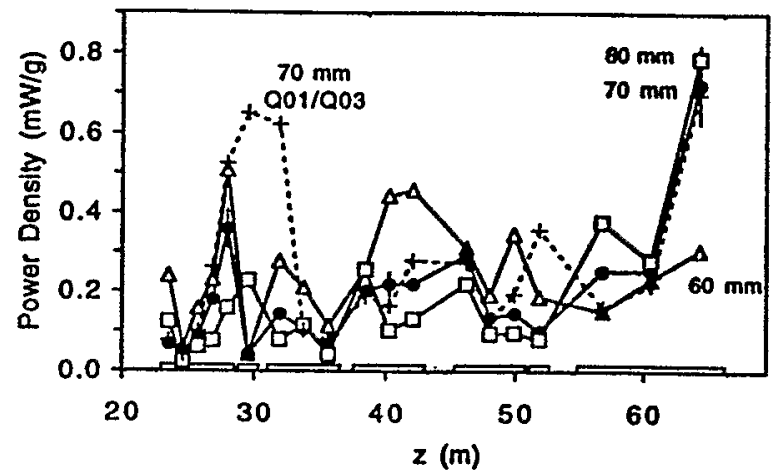

Figure 3: $P_{\max }$ vs $z$ for main quadrupoles of three $d_{\text {coil }}$ with $85 \mathrm{~mm}$ trim quadrupoles, and $70 \mathrm{~mm}$ main with $70 \mathrm{~mm}$ trim quadrupoles. The data are plotted at the $z$ values for the $70 \mathrm{~mm}$ quadrupoles to ease comparison.

thickness, making this option impractical except possibly with $80 \mathrm{~mm}$ magnets. The longitudinal power distribution is shown in Table 4 for $70 \mathrm{~mm}$ quadrupoles with $10 \sigma \mathrm{ab}-$ sorbers. Averaged over each element, the power density varies from $3 \mathrm{~W} / \mathrm{m}(\mathrm{Q} 2 \mathrm{a})$ to $10 \mathrm{~W} / \mathrm{m}(\mathrm{Q} 01)$. However, the variation within one magnet can be as large as a factor of 8 ( $Q 1$ with uniform diameter absorbers).

\section{CONCLUSIONS}

Energy deposited in the superconducting magnets is an important issue in the overall design of the LHC IRs. Reducing $P_{\max }$ to an acceptable level requires the use of internal absorbers at least 5-6 mm thick. Quadrupoles with $d_{\text {coil }}=70 \mathrm{~mm}$, the current baseline design, are large enough to accommodate such liners and leave a $10 \sigma$ physical aperture. A larger $d_{\text {coil }}$ would allow use of a thicker absorber, greater physical aperture for the same absorber thickness, or possibly cooling the absorber at a higher temperature than the magnet. $P_{\max }$ in D1 at the high luminosity IRs is unacceptably large if dipoles of the baseline length are used. However, here the dipoles can be moved farther apart reducing their length by up to a factor of 3 or allowing the use of conventional magnets.
Table 3: Total deposited power (W).

\begin{tabular}{|l||r|r|r|r|r|r|}
\hline $\begin{array}{l}d_{\text {coil }(m m)} \\
\text { Absorber }(\sigma)\end{array}$ & $\begin{array}{r}70 \\
\text { none }\end{array}$ & $\begin{array}{r}70 \\
10\end{array}$ & $\begin{array}{r}70 \\
10 \\
\text { unif }\end{array}$ & $\begin{array}{r}70 \\
8\end{array}$ & $\begin{array}{r}60 \\
10\end{array}$ & $\begin{array}{r}80 \\
10\end{array}$ \\
\hline \hline Quadrupoles & 115 & 82 & 86 & 66 & 98 & 69 \\
Absorbers & - & 61 & 52 & 73 & 37 & 78 \\
Total & 115 & 143 & 138 & 139 & 135 & 147 \\
\hline D1 & 45 & 26 & 34 & 19 & 24 & 25 \\
Absorber & - & 10 & 16 & 15 & 12 & 9 \\
Total & 45 & 36 & 50 & 34 & 36 & 34 \\
\hline
\end{tabular}

Table 4: Total power $(W)$ deposited in each magnet for $70 \mathrm{~mm}$ quadrupoles with $10 \sigma$ absorbers.

\begin{tabular}{|l||r|r|r|r|r|r|}
\hline & Q1 & Q01 & Q2a & Q2b & Q3 & Q03 \\
\hline \hline Magnet & 15 & 6 & 13 & 17 & 26 & 4 \\
Absorber & 18 & 10 & 5 & 9 & 13 & 7 \\
Total & 33 & 16 & 18 & 26 & 39 & 11 \\
\hline
\end{tabular}

\section{ACKNOWLEDGEMENTS}

We would like to thank P. Limon, R. Ostojic, K. Potter and T. Taylor for useful discussions. This work was supported in part by the U.S. Department of Energy.

\section{REFERENCES}

[1] "The Large Hadron Collider Conceptual Design," CERN/AC/95-05(LHC), 1995, P. Lefevre and T. Pettersson, editors.

[2] K. Eggert and A. Morsch, "Particle Losses in the LHC Interaction Regions," CERN AT/93-17 (DI), 1993; A. Morsch, "Local Power Distribution from Particle Losses in the LHC Inner Triplet Magnet Q1," CERN AT/94-06 (DI), 1994.

[3] A. Morsch, R. Ostojic, T.M. Taylor, "Progress in the Systems Design of the Inner Triplet of $70 \mathrm{~mm}$ Aperture Quadrupoles for the LHC Low-beta Insertions," Proc. 4th European Part. Accel. Conf., London, England, 1994.

[4] N. V. Mokhov, “Accelerator/Experiment Interface at Hadron Colliders: Energy Deposition in the $\mathbb{R}$ Components and Machine Related Background to Detectors", Fermilab-Pub94/085, 1994.

[5] L. Bumod, et al., "Thermal Modelling of the LHC Dipoles Funcitioning in Superfluid Helium," Proc. 4th European Part. Accel. Conf., London, England, 1994.

[6] R. Ostojic and T.M. Taylor, "Proposal for and Improved Optical and Systems Design of the LHC Low- $\beta$ Triplets," CERN AT/94-38.(MA), 1994.

[7] R. Ostojic, T.M. Taylor and G.A. Kirby, "Design and Construction of a One-Metre Model of the $70 \mathrm{~mm}$ Aperture Quadrupole for the LHC Low- $\beta$ Insertions," Proc. 13th Int. Conf. on Mag. Tech. (MT13), Victoria, Canada, 1993.

[8] P. Aurenche, et al., "DTUjeT93", Comput. Phys. Commun., 83, p. 107, 1994.

[9] N. V. Mokhov, "The MARs Code System User's Guide, Version 13(95)", Fermilab-FN-628, 1995. 
$\therefore-$

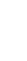




\title{
AMPLITUDE DEPENDENT TUNE SPREAD AND FIELD ERRORS OF SUPERCONDUCTING LOW- $\beta$ QUADRUPOLES
}

\author{
R. Ostojic and T. M. Taylor \\ LHC Division, CERN, Geneva, Switzerland
}

\begin{abstract}
The nonlinear effects in the low- $\beta$ insertions are studied on the basis of the amplitude dependent tune spread. Several methods of estimating the tune spread in superconducting low- $\beta$ quadrupoles are compared. The main feature of these magnets is that the random errors dominate the multipole spectrum. The proposed methods allow an analysis of the final focus layout in the early stages of the insertion design, and point to the critical magnets and the dominant multipole errors.
\end{abstract}

\section{INTRODUCTION}

The large $\beta$-functions in the low- $\beta$ insertions which are associated with small values of $\beta^{*}$, chosen for optimising the luminosity, are generally considered a limiting factor of hadron collider performance. High- $\beta$ conditions inside the final focus quadrupoles are the source of chromatic and amplitude dependent effects. While both are related to the precise layout of the low- $\beta$ section, the field quality of the low- $\beta$ quadrupoles drives non-linear resonances, which together with the beam-beam collisions, limit the lifetime of the colliding beams. In order to estimate the relevance of the large amplitude motion, the contribution of each low- $\beta$ quadrupole should be considered by taking into account the details of the local optics, in particular of the variation of the $\beta$-function and of the central orbit inside the quadrupoles, and of the possible asymmetries of the layout around the collision point. It is also important to study the layout in the early stages of the design, and to determine those features of the low- $\beta$ quadrupoles which contribute mostly to exciting the non-linearities. An important indicator in this respect is the amplitude dependent tune spread.

In this report we propose a method of estimating the amplitude dependent tune spread of a low- $\beta$ triplet of superconducting quadrupoles, in which the random errors typically dominate the multipole spectrum. These quadrupoles cannot be treated as thin lenses both because of their length and gradient, and because of the displaced beam trajectories arising from finite crossing angles. Furthermore, the optical functions vary considerably over short lengths, comparable to the extension of the quadrupole end field, where the systematic multipoleerrors are compensated on the average. With modest computational effort, dominant features of the triplet layout can be determined, and tolerance limits on the multipole errors can be derived.

\section{AMPLITUDE DEPENDANT TUNE SPREAD IN PERTURBATION THEORY}

The long-term particle motion in non-linear magnetic fields is an area of extensive studies. The long term dynamic aperture is correlated to the tune spread of the beam, with the value of around $\Delta Q=0.015$, chosen for the LHC [1], considered as the upper limit for a hadron collider. The dominant part of the tune spread budget is attributed to the headon and parasitic beam-beam collisions. While only a small fraction is related to the single particle motion (typically $\Delta Q=0.005$ ), mostly due to large amplitude oscillations in the low- $\beta$ insertions, the amplitude dependent tune spread beyond this level clearly limits the dynamic aperture.

We base our approach of estimating the amplitude dependent tune spread on perturbation theory, where the nonlinear terms of the Hamiltonian are treated as perturbations to the well known linear motion [2]. To first order, the tune shift is obtained by averaging the phase independent part of the perturbation around the ring. As the perturbation is directly related to the multipole spectrum of the guide field, customarily represented as :

$$
B_{y}+i B_{x}=B_{0} \sum_{n=1}\left(b_{n}+i a_{n}\right)\left(\frac{x+i y}{r_{0}}\right)^{n-1}
$$

the contribution of the $k$-th multipole error to the tune shift may be written in terms of the optics and multipole field errors of each magnet in a given section of the low- $\beta$ insertion:

$$
\delta Q_{x}=\int \frac{d s}{2 \pi \rho} \beta_{x} \sum_{n=1}^{10}\left(C_{n}^{x, b} b_{n}+C_{n}^{x, a} a_{n}\right)
$$

The most important coefficients for the bending plane are :

$$
\begin{aligned}
& C_{4}^{x, b}=(1-\delta)\left(1.5 \Delta^{2}+\frac{J_{1}}{4}\right) \\
& C_{5}^{x, b}=(1-\delta) \Delta_{x} J_{1} \\
& C_{6}^{x, b}=(1-\delta)\left(2.5 \Delta^{2} J_{1}+\frac{J_{2}}{6}\right)
\end{aligned}
$$

where:

$$
\begin{aligned}
\Delta_{x} & =x_{c}+D_{x} \delta \\
\Delta_{y} & =y_{c}+D_{y} \delta \\
\Delta^{2} & =\Delta_{x}^{2}-\Delta_{y}^{2} \\
J_{1} & =3\left(J_{x} \beta_{x}-2 J_{y} \beta_{y}\right) \\
J_{2} & =\frac{15}{2}\left(J_{x}^{2} \beta_{x}^{2}-6 J_{x} \beta_{x} J_{y} \beta_{y}+3 J_{y}^{2} \beta_{y}^{2}\right)
\end{aligned}
$$


luminosity insertions [1]. The "Gaussian beam" tune spread is considered a baseline method, but is itself time consuming and inappropriate for examining a large number of different situations. A very precise upper limit is obtained by using the " $4 \sigma$ " method, which is presented in Fig. 1 by its "four point" footprint $\left(\left(J_{x}, J_{y}\right)=(0,0),(0,4),(4,0),(4,4)\right)$. The non-zero average tune shift in these two cases is due to the fact that all random errors are considered, including the strong $b_{3}$ term which contributes to the average tune shift but not to the tune spread. The tune spread obtained by the "rms" and the "maximum error" methods, are on the contrary centred at the tune shift produced by the small systematic errors. The "rms" tune spread is by a factor of 1.6 larger than the " $4 \sigma$ " value and requires the smallest computational effort. However, we consider the " $4 \sigma$ " method as the most appropriate as it consistently gives good results within short computational time. The "maximum error" case is larger by a factor of 1.7.

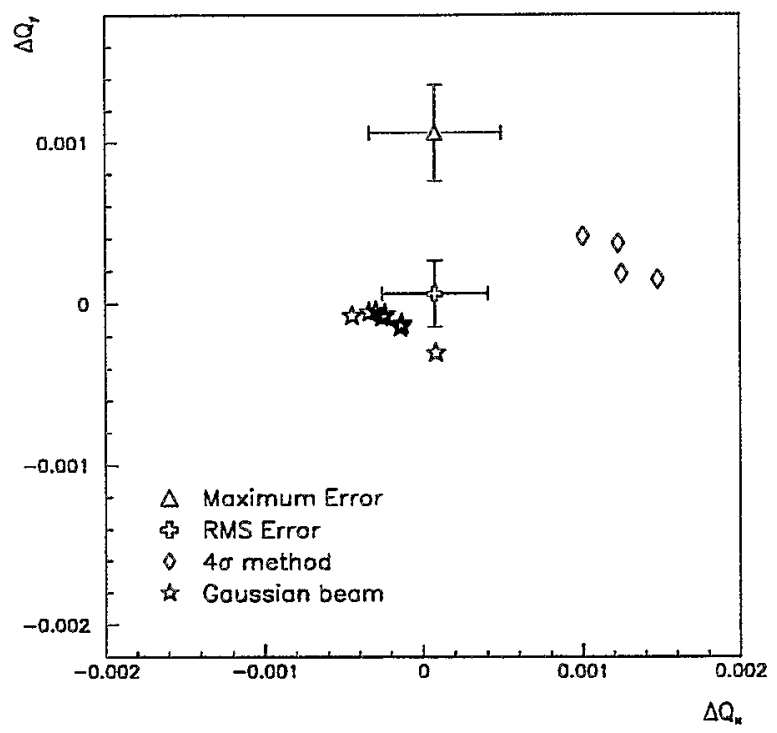

Figure 1: Comparison of the tune spread estimates for the LHC low $\beta$ triplet. For clarity, the "maximum error" tune spread has been shifted by 0.001 in $\Delta Q_{y}$.

\section{ANALYSIS OF LOW- $\beta$ TRIPLET LAYOUTS}

The major advantage of a simple method of estimating the amplitude dependent tune spread is that a number of configurations can be studied in the early stages of the insertion design without resorting to time consuming tracking studies. In the case of the LHC low- $\beta$ quadrupoles [3], the application of this method gave the following results:

- The tune spread of the low- $\beta$ insertion is dominated by the quadrupoles sitting in regions of peak $\beta$-values, which contribute $90 \%$ of the total tune spread. The offcentred central orbit $(\leq 4 \sigma)$ increases the tune spread by a factor of 2.5 . In the LHC, changes in $\beta^{*}$ influence the tune spread twice as much as changes of the crossing angle.

- The multipoles which contribute most to the tune spread are the octupole and decapole random, and dodecapole systematic and random errors. The errors of quadrupole ends, in particular the $b_{6}$ component of the lead end, contribute to about half of the total tune spread. These errors are systematic, and can be compensated by passive or active methods.

- By choosing the side on which to put magnet connections, the low $-\beta$ triplet can be made such that the two LHC beams have the same tune spread. The value of the tune spread is reduced from $\Delta Q=510^{-4}$ to $310^{-4}$ by the correct choice of the position of the connection side.

- Assuming the "maximum error" case and four low- $\beta$ insertions tuned at a $\beta^{*}$ of $0.5 \mathrm{~m}$, the LHC tune spread is by a factor 1.6 below the tentative limit of 0.005 .

The last result indicates that the dynamic aperture of the LHC at top energy should not be limited by the random multipoles. This is confirmed by recent tracking studies [4], which give a dynamic aperture of $10 \sigma$ in physics conditions, on the edge of the "good field region" of the $70 \mathrm{~mm}$ aperture low- $\beta$ quadrupoles.

\section{CONCLUSIONS}

We have compared several methods of estimating the amplitude dependent tune spread in superconducting low- $\beta$ quadrupoles. The approach addresses the main feature of the multipole spectrum of these magnets, i.e. the dominance of the random errors. Furthermore, the details of the local optics, in particular the rapid variation of the $\beta$-function and central orbit deviation, and the asymmetries of the insertion optics are taken into account. The method allows to determine the critical quadrupoles and multipole errors, to investigate the role of magnet orientation and connections, and to set limits on multipole error tolerances.

\section{REFERENCES}

[1] The LHC Study Group, The Large Hadron Collider, Conceptual Design, CERN/AC/95-05.

[2] S. Heifets, “Advanced Non-linear Theory", Physics of Particle Accelerators, AIP Conf. Proc. 184, New York 1989.

[3] R. Ostojic T.M. Taylor and G.A. Kirby, "Design and Consiruction of a One-Metre Model of the $70 \mathrm{~mm}$ Aperture Quadrupole for the LHC Low- $\beta$ Insertions", Proc. 13th Int. Conf. on Magnet Technology (MT13), Victoria, Canada, September 1993, LHC Note 245.

[4] A. Faus-Golfe, A. Verdier, "Dynamic Aperture Limitations of the LHC in Physics Conditions due to Low- $\beta$ Insertions". Proc. of this Conference. 
luminosity insertions [1]. The "Gaussian beam" tune spread is considered a baseline method, but is itself time consuming and inappropriate for examining a large number of different situations. A very precise upper limit is obtained by using the " $4 \sigma$ " method, which is presented in Fig. 1 by its "four point" footprint $\left(\left(J_{x}, J_{y}\right)=(0,0),(0,4),(4,0),(4,4)\right)$. The non-zero average tune shift in these two cases is due to the fact that all random errors are considered, including the strong $b_{3}$ term which contributes to the average tune shift but not to the tune spread. The tune spread obtained by the "rms" and the "maximum error" methods, are on the contrary centred at the tune shift produced by the small systematic errors. The "rms" tune spread is by a factor of 1.6 larger than the " $4 \sigma$ " value and requires the smallest computational effort. However, we consider the " $4 \sigma$ " method as the most appropriate as it consistently gives good results within short computational time. The "maximum error" case is larger by a factor of 1.7.

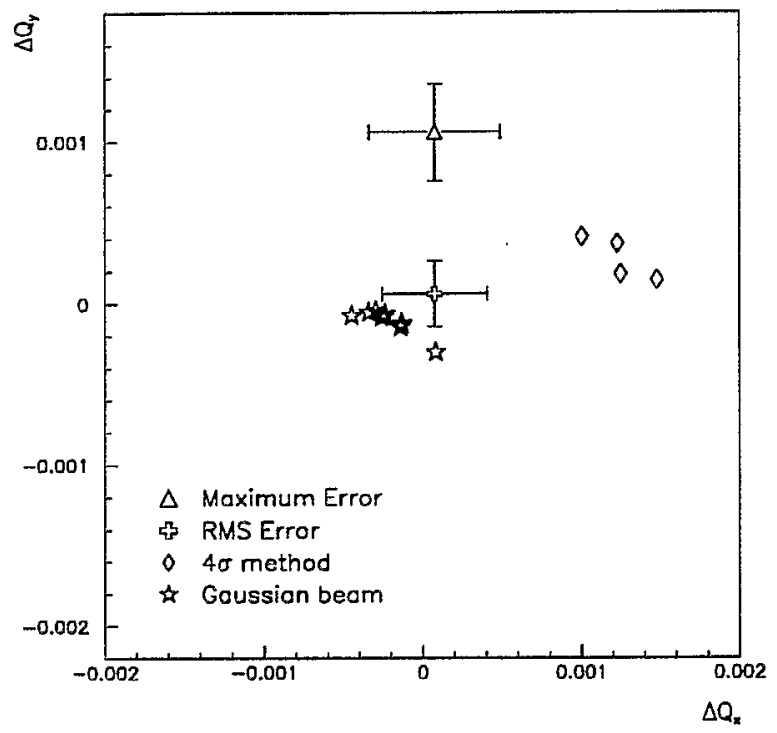

Figure 1: Comparison of the tune spread estimates for the LHC low- $\beta$ triplet. For clarity, the "maximum error" tune spread has been shifted by 0.001 in $\Delta Q_{y}$.

\section{ANALYSIS OF LOW- $\beta$ TRIPLET LAYOUTS}

The major advantage of a simple method of estimating the amplitude dependent tune spread is that a number of configurations can be studied in the early stages of the insertion design without resorting to time consuming tracking studies. In the case of the LHC low- $\beta$ quadrupoles [3], the application of this method gave the following results:

- The tune spread of the low- $\beta$ insertion is dominated by the quadrupoles sitting in regions of peak $\beta$-values, which contribute $90 \%$ of the total tune spread. The offcentred central orbit $(\leq 4 \sigma)$ increases the tune spread by a factor of 2.5 . In the LHC, changes in $\beta^{*}$ influence the tune spread twice as much as changes of the crossing angle.

- The multipoles which contribute most to the tune spread are the octupole and decapole random, and dodecapole systematic and random errors. The errors of quadrupole ends, in particular the $b_{6}$ component of the lead end, contribute to about half of the total tune spread. These errors are systematic, and can be compensated by passive or active methods.

- By choosing the side on which to put magnet connections, the low- $\beta$ triplet can be made such that the two LHC beams have the same tune spread. The value of the tune spread is reduced from $\Delta Q=510^{-4}$ to $310^{-4}$ by the correct choice of the position of the connection side.

- Assuming the "maximum error" case and four low- $\beta$ insertions tuned at a $\beta^{*}$ of $0.5 \mathrm{~m}$, the LHC tune spread is by a factor 1.6 below the tentative limit of 0.005 .

The last result indicates that the dynamic aperture of the LHC at top energy should not be limited by the random multipoles. This is confirmed by recent tracking studies [4], which give a dynamic aperture of $10 \sigma$ in physics conditions, on the edge of the "good field region" of the $70 \mathrm{~mm}$ aperture low- $\beta$ quadrupoles.

\section{CONCLUSIONS}

We have compared several methods of estimating the amplitude dependent tune spread in superconducting low- $\beta$ quadrupoles. The approach addresses the main feature of the multipole spectrum of these magnets, i.e. the dominance of the random errors. Furthermore, the details of the local optics, in particular the rapid variation of the $\beta$-function and central orbit deviation, and the asymmetries of the insertion optics are taken into account. The method allows to determine the critical quadrupoles and multipole errors, to investigate the role of magnet orientation and connections, and to set limits on multipole error tolerances.

\section{REFERENCES}

[1] The LHC Study Group, The Large Hadron Collider; Conceptual Design, CERN/AC/95-05.

[2] S. Heifets, "Advanced Non-linear Theory", Physics of Particle Accelerators, AIP Conf. Proc. 184, New York 1989.

[3] R. Ostojic T.M. Taylor and G.A. Kirby, "Design and Construction of a One-Metre Model of the $70 \mathrm{~mm}$ Aperture Quadrupole for the LHC Low- $\beta$ Insertions", Proc. 13th Int. Conf. on Magnet Technology (MT13), Victoria, Canada, September 1993, LHC Note 245.

[4] A. Faus-Golfe, A. Verdier, "Dynamic Aperture Limitations of the LHC in Physics Conditions due to Low- $\beta$ Insertions", Proc. of this Conference. 


\title{
SUPERCONDUCTING SEXTUPOLE CORRECTOR MAGNET FOR THE LHC MAIN DIPOLES
}

\author{
Jukka Salminen, Albert Ijspeert, LHC Division, CERN, Geneva, Switzerland \\ Avinash Puntambekar, CAT, Indore, India
}

\section{Abstract}

Each LHC main dipole will be equipped with small sextupole corrector magnets with a field strength of $1970 x^{2} \mathrm{~T} / \mathrm{m}^{2}$ and a magnetic length of $100 \mathrm{~mm}$ designed to correct the sextupole field errors. The paper presents a cosine- $\theta$ type of design where much emphasis has been put on the cost reduction because these magnets have to be made in a large series of some $\mathbf{2 5 0 0}$ pieces. We describe the design of a two layer coil which can be wound automatically. The winding starts in the middle of the wire with the only joggle, the layer jump, which is housed in a corresponding groove in the end of the central island. The two layers are wound simultaneously tuming in opposite directions to find their position without the need of local tooling. The coil ends are closely packed and need no end spacers. The 18 pole perturbation introduced by the ends is corrected by the position of the coil block in the straight part. The yoke is made of iron laminations of the "Scissors type" which transmit the pre-stress from the outer aluminium shrink ring to the coil. This allows the iron to be close to the coil for field enhancement and also boosts the pre-stress in the coil due to the cool down contractions. The paper describes the experience with the magnet construction and gives the first test results.

\section{INTRODUCTION}

\subsection{General}

In the LHC ring each bore of the main bending dipole is equipped with a superconducting sextupole (MCS) and decapole (MCD) corrector magnet to correct systematic sextupole and decapole field errors in the dipole field. Sextupole correctors are placed at one end of the dipoles and decapoles at the opposite end. In the first generation sextupole comector design, coils were wound around a copper central island in eight layers cach consisting of fourteen tums [1]. This coil design proved to be laborious and expensive to manufacture. since after each turn the cable must be twisted to be able to jump to the next turn, and after each layer to do a layer jump. During the winding each turn must be clamped by special tooling to prevent unwinding of the coil. The new design drastically reduces the time to make a magnet. In this paper will be described only the design and the manufacturing of the sextupole magnet however similar design principles will be used for the decapole and octupole magnets. Six of this type of sextupole and docapole and four octupole magnets will be built at CERN by beginning of April 1997.

\section{Design Criteria}

The design criteria of the magnets are given by the beam dynamics and the available space in the dipole ends. The main parameters are given in the Table 1 . In the LHC ring the corrector power supplies will be of 600A type.

\begin{tabular}{|lr|}
\hline Nominal strength & $1970 x^{2} \mathrm{~T} / \mathrm{m}^{2}$ \\
Magnetic / Overall length & $104 / 150 \mathrm{~mm}$ \\
Nominal current & $625 \mathrm{~A}$ \\
Peak field in the coil 3D & $2.12 \mathrm{~T}$ \\
Self inductance & $0.672 \mathrm{mH}$ \\
Inner / Outer diameter of the coil & $58 / 61 \mathrm{~mm}$ \\
Inner / Outer diameter of the yoke & $66 / 90 \mathrm{~mm}$ \\
Inner / Outer diameter of shrink ring & $89.88 / 100 \mathrm{~mm}$ \\
Cable dimensions bare & $1.13 \times 0.606 \mathrm{~mm}$ \\
Cu/Sc ratio & 1.6 \\
Insulation thickness (PVA) & $0.06 \mathrm{~mm}$ \\
Turns per coil & $2 \times 13$ \\
Working temperature & $1.9 \mathrm{~K}$ \\
\% on the load line 1.9/4.2 K & $62.9 / 47.5$ \\
\hline
\end{tabular}

Table 1. Magnet parameters

\section{MAGNETIC DESIGN}

\subsection{General}

The magnetic design aimed for an economic two layer coil. In the design a combination of 2D and 3D modelling has been used. 3D models have been created using the ROXIE program, which uses Biot-Savart on the line currents [2]. The surrounding iron yoke can only be modelled with linear or infinite permeability. The induction of the iron remains well below the saturation level up to nominal operation level, which was checked with the POISSON program in 2D. ROXIE is not able to calculate 3D peak fields in a coil surrounded by iron, the 2D peak field in the coil is calculated with and without iron and the same ratio is assumed to be valid in 3D. 

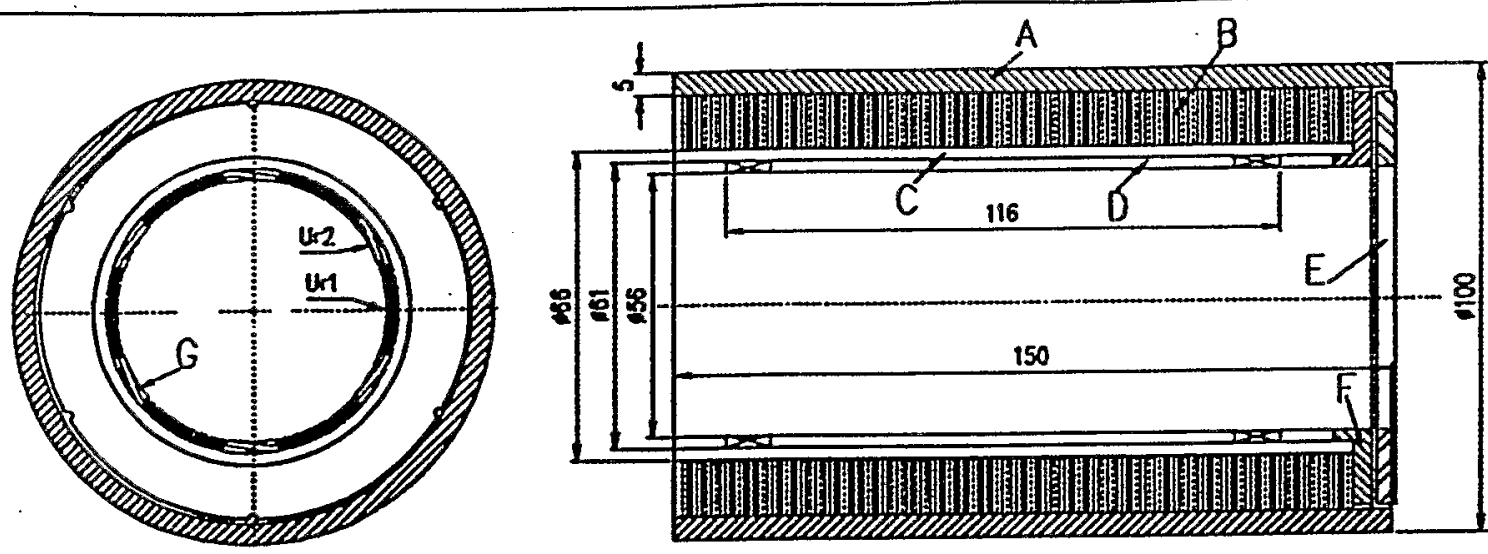

Figure 1. MCS magnet A: Shrinking ring B: Scissor laminations C: Glass fibre bandage D: Coils E: End cover . F: End flange for the connections G: Layer jump in one end of the central island

\subsection{Coil optimisation}

By bringing the surrounding yoke close to the coils for field enhancement, it was possible to reach the required field strength by using a two layer coil. The iron boosts the field by $48.5 \%$. The coil ends are designed without end spacers to allow for automatic winding. The eighteen pole perturbation induced by the coil ends was compensated by the position of the coil block in the straight part (Table 2). An existing wire was chosen because it's availability. In the LHC ring 154 of these magnets are connected in series and in case of a quench, most of the energy of the 153 other magnets is absorbed in the quenching magnet. In order to survive a quench, for the final design the amount of the copper in the wire will be increased by $40 \%$, i.e. the Cu/Sc ratio increases from 1.6 to 2.25 [3].

\begin{tabular}{|ccc|}
\hline B3 $[\mathrm{Tm}]$ & $\mathrm{B} 9 / \mathrm{B} 3\left[\times 10^{-4}\right]$ & $\mathrm{B} 15 / \mathrm{B} 3\left[\times 10^{-4}\right]$ \\
-0.0206249 & 0.01978 & 0.000417 \\
\hline
\end{tabular}

Table 2. Integrated harmonics at radius of $10 \mathrm{~mm}$

\section{MECHANICAL DESIGN}

\subsection{Central island material}

Glass fibre reinforced epoxy (G11) was chosen for central islands and end spacers material, since it is integrated contraction factor $260 * 10^{\circ}$, in the longitudinal direction between 293 and $4.2 \mathrm{~K}$, is close to that of the cables $295^{*} 10^{5}$. In addition to which no ground insulation is needed. The end filling pieces and central islands were machined from tubes of this material.

\section{Scissor laminations}

The iron yoke is composed of so called scissor laminations, which transmit the pre-stress from the outer aluminium shrink ring into the coils [4]: Two slightly $e c-$ centric laminations, which press from opposite directions on the glass fibre bandage around the coils, form a pair of "scissors". Over the length of the magnet, successive pairs of laminations are rotated by $60^{\circ}$. As the lamination thickeness is $1 \mathrm{~mm}$, every $6 \mathrm{~mm}$ pressure is applied in the same angular position.

\subsection{FEM-model}

A 2D FEM-model has been created, to define stresses in the structure. A $30^{\circ}$ sector of the magnet has been modelled using the ANSYS code. The eccentric contact of the scissor laminations to the aluminium shrink ring and the fibre glass bandage has been simulated by using contact elements. Table 5 presents the calculated azimuthal stresses in the coil and in the aluminium cylinder during different load steps.

\begin{tabular}{|lccc|}
\hline & $293 \mathrm{~K}$ & $4.2 \mathrm{~K}$ & $4.2 \mathrm{~K}(1000 \mathrm{~A})$ \\
$\sigma$ coil max. & -21 & -27 & -22 \\
$\sigma$ coil min. & -49 & -68 & -74 \\
$\sigma$ cyl max. & 56 & 107 & 108 \\
$\sigma$ cyl min. & -7 & 21 & 28 \\
Rad disp Ur1 & -0.020 & -0.139 & -0.133 \\
Rad disp Ur2 & -0.0334 & -0.168 & -0.166 \\
\hline
\end{tabular}

Table 3. Azim. stresses in coil and shrink ring [MPa] and radial displacements of the coil [mm] (Fig 1). Radial interference between yoke and shrink ring is $0.06 \mathrm{~mm}$

\section{FABRICATION}

\subsection{Coil winding}

The two layer coil (double pancake) design allows to start winding in the middle of the coil with the only joggle, the layer jump, which has been housed in a groove in the end of the central island. The two layers are wound simultaneously in opposite directions to find their position automatically not needing the clamps normally used to keep the wire in it's plane. The wire is continuously wetted during the winding process by epoxy (Fig. 2). To produce compact coils a constant winding tension of $35 \mathrm{MPa}$ was maintained in the metal 
cross section of the cable. Although the wire is thin, when bent over the end of the central post, it has a tendency to bend away from the central post in the straight part, due to its bending stiffness. If the wires are then pushed towards the central island after the whole coil is wound, most of the winding tension will be lost. The situation can be improved, by counter bending the wire around a small pulley, which deforms the wire so that it naturally pushes itself towards the central post. In addition to counter bending, before winding the central island is pre-compressed in the longitudinal direction by $0.1 \mathrm{~mm}$. This is done by applying a load of $350 \mathrm{~N}$ the ends, and then locking the central island in place by screws. After the winding the locking screws are loosened so that the pre-stressed central island and the surrounding coil find an cquilibrium. After the winding the end fillers are positioned and the coil is clamped in the curing mould, which is doweled through the central island on the winding mandrel. Finally the coil is heated in the oven in order to cure the epoxy. Winding and impregnation take approximately two hours per coil using a simple hand driven machine.

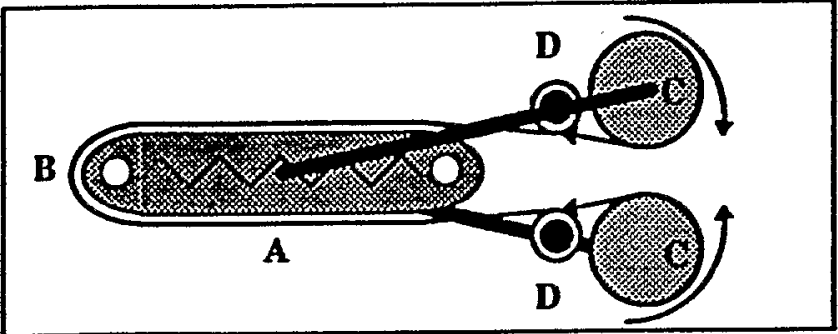

Figure 2. Winding A: Pre-compressed central island B: Layer jump C: Wire reels D: Counter bending pulleys

\subsection{Assembly of the magnet}

The impregnated coils are positioned by dowel pins around an assembly mandrel and a glass fibre-epoxy end flange is placed at one end of the coils for the series connections. A prepreg glass fibre cloth is wrapped around the coils whereupon it is clamped and cured in a oven. After turning the bandage, holes are drilled in the positions of the dowel pins, and these pins are pushed into the hollow mandrel which is then pulled out. The series connections are made by soldering the wires side by side in grooves on the end flange over a length of $35 \mathrm{~mm}$. The connections are kept in place by a glass fibre cover plate which is screwed on the end flange. The yoke laminations are assembled around the glass fibre bandage as deseribed in the section 3. To facilitate the slipping on of the heated aluminium shrink ring over the laminations, a slightly conical Teflon hat is mounted at the end of the magnet.

\section{TEST RESULTS}

The training test of the magnet was done at $4.2 \mathrm{~K}$ in Madrid, by CEDEX. The first quench of the magnet was at 734 A which is well above the nominal operation level (Fig. 3). After a heat cycle to room temperature the magnet showed very little re-training i.e. the first quench occurred at $934 \mathrm{~A}$. The current was measured by clamp meters around the bus bars with an accuracy of $\pm 20 \mathrm{~A}$. Voltage staps were installed over the poles in order to record the quench voltages. Also the current decay was measured in order to calculate the MIIT's and hence define the hot spot temperatures which proved to be at maximum around $130 \mathrm{~K}$.

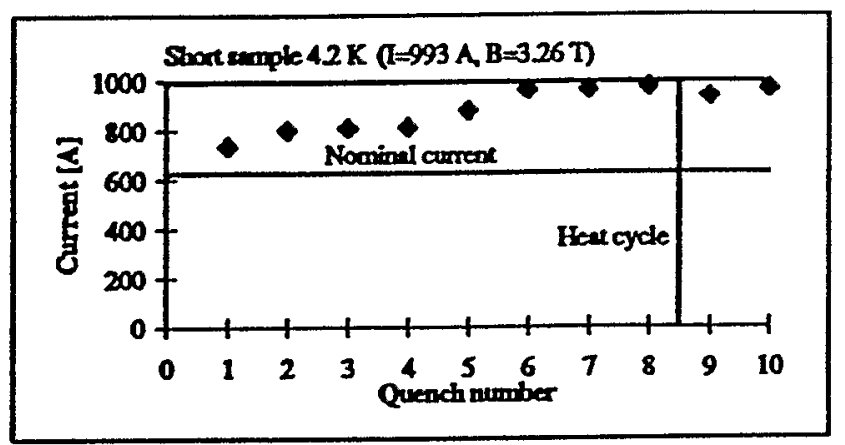

Figure 3. Training of the magnet at $4.2 \mathrm{~K}$.

\section{CONCLUSIONS}

A second generation cosine- $\theta$ type of superconducting sextupole corrector magnet (MCS) has been designed and built at CERN. A training test has been done at 4.2 $\mathrm{K}$ by CEDEX. By using scissor laminations, it has been possible to bring the iron close to the coils for maximum field enhancement. Since the iron boosts the field by $49 \%$, the required field strength of $0.197 \mathrm{~T}$ at $10 \mathrm{~mm}$ radius, is obtained by two layer coil. The aim of the two layer coil design has been to allow for an easy automatization of the winding process.

\section{ACKNOWLEDGEMENT}

The authors acknowledge J. Perez and J. Mazet who carried out numerous winding tests and built the magnet as well as D. Regin who shared his experience. We also acknowledge the help of our colleagues in CAT, especially M. Karmakar and R. Adbol. Finally we would like to thank L. Garcfa-Tabares and his team in CEDEX for testing the magnet.

\section{REFERENCES}

[1] D. Bayham, R. Coombs, A. Ijspeert, R. Perin, "Design of Supenconducting Corrector Magnets for LHC IEEE Trans. on Magnetics pp. 1823-1826, Vol 30, No 4, July 1994

[2] S. Russenschuck, "A computer program for the Design of the Superconducting Accelerator Magnet", Proc. of ACES 95, Monterey, CA, USA

[3] D. Hagedom, F. Rodrigues-Mateos, R. Schmidt, "Protection of LHC Superconducting Corrector and Insertion Magnets", In this conference

[4] A. Ijspeert, J. Salminen, "Superconducting Coil Compression by Scissors Laminations". In this conference 
0
$-\quad: \quad-$

? 
ap102.txt

Fri Jun 21 08:20:50 1996

1

From peggs@acnsun10.rhic.bnl.gov Thu Jun 20 12:45 EDT 1996

To: manning@acnsun10.rhic.bnl.gov

Subject: RHIC/AP/102

Content-Type: text

Content-Length: 250

Pam,

here's a list of people to receive the full version of RHIC/AP/102:

myself ( 3 copies total, please)

-animesh jain

Lames gupta

$t$ peter wanderer

ie wei

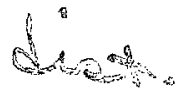

Dejan trbojevic

pat thompson

mike harrison

$6 / 21 / 96$

good enough for starters.

thanks,

steve

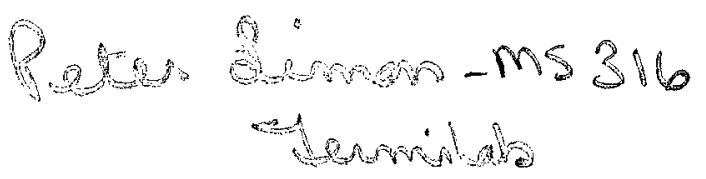


IF YOU ARE INTERESTED IN RECEIVING ANY OR ALL OF THESE REPORTS PLEASE CONTACT PAM MANNING X4072, EMAIL: MANNING1@BNL.GOV

\section{EPAC/LHC Magnet Papers}

- G. Ambrosio, F.M. Ametrano, F. Broggi, A. Ferrari and L. Rossi, "Stability of a NbSn Low-Beta Quadrupole in the LHC Radiation Environment"

- G. Ambrosio, G. Bellomo and L. Rossi, "A 300 T/m NbSn Quadrupole for the LowBeta Insertions of the LHC"

- N. Andreev, K. Artoos, L. Bottura, G. Kirby, D. Leroy, L. Oberli, J. Ostler, D. Perini, A. Poncet, F. Rodrigues-Mateos, S. Russenschuck, T. Siambanis, N. Siegel, A. Siemko, D. Tommasini, G. Trinquart, I. Vanekov, L. Walckiers, W. Weterings, "The $1 \mathrm{~m}$ Long Single Aperture Dipole Coil Test Program for LHC"

- R. Bossert, S. Feher, S.A. Gourlay, T. Heger, J. Kerby, M.J. Lamm, P.J. Limon, P.O. Mazur, T. Nicol, F. Nobrega, D. Orris, J.P. Ozelis, T. Peterson, P. Schlabach, J. Strait, J. Tompkins, A. Zlobin, A. Lietzke, A.D. McInturff, R. Scanlan, "Low Temperature Quench Performance of Fermilab Low- $\beta$ Insertion Quadrupoles"

- L. Bottura, A. Faus-Golfe, L. Walckiers, R. Wolf, "Field Quality of the Main Dipole Magnets for the LHC Accelerator"

- A. Faus-Golfe and A. Verdier, "Dynamic Aperture Limitations of the LHC in Physic Conditions due to Low-Beta Insertions"

- Ramesh Gupta, "Field Quality in Superconducting Magnets for Large Particle Accelerators"

- R.C. Gupta, R. Bossert, S.A. Gourlay, T. Heger, Y. Huang, J. Kerby, M.J. Lamm, P.J. Limon, P.O. Mazur, F. Nobrega, J.P. Ozelis, G. Sabbi, J. Strait, A.V. Zlobin, S. Caspi, D. Dell'orco, A.D. McInturff, R.M. Scanlan, J.M. Van Oort, "Design of a High Gradient Quadrupole for the LHC Interaction Regions"

- A. Ijspeert, J. Salminen, "Superconducting Coil Compression by Scissor Laminations"

- A. Ijspeert, R. Perin, E. Baynham, P. Clee, R. Coombs, D. Evans, M. Begg, D. Landgrebe, "Construction and Tests of a Model of the LHC Superconducting Corrector Magnet MDSBV"

- M. Karppinen, S. Russenschuck A. Ijspeert, "Automated Design of a Correction Dipole Magnet for LHC"

- N. Mokhov, J. Strait, "Optimization of the LHC Interaction Region with Respect to Beam-Induced Energy Deposition"

- R. Ostojic and T.M. Taylor, "Amplitude Dependent Tune Spread and Field Errors of Superconducting Low- $\beta$ Quadrupoles"

- J. Salminen, A. Ijspeert, A. Puntambekar, "Superconducting Sextupole Corrector Magnet for the LHC Main Dipoles" 